\title{
COMPARATIVE ANALYSIS OF ELECTRODYNAMIC TOROIDAL RADIATION SHIELDING CONFIGURATIONS
}

\author{
A Thesis \\ presented to \\ the Faculty of California Polytechnic State University,
}

San Luis Obispo

\author{
In Partial Fulfillment \\ of the Requirements for the Degree \\ Master of Science in Aerospace Engineering
}

by

Max Rosenberg

December 2018 
(C) 2018

Max Rosenberg

ALL RIGHTS RESERVED 


\section{COMMITTEE MEMBERSHIP}

TITLE: Comparative Analysis of Electrodynamic Toroidal Radiation Shielding Configurations

AUTHOR: Max Rosenberg

DATE SUBMITTED: December 2018

COMMITTEE CHAIR: Kira Abercromby, Ph.D.

Associate Professor of Aerospace Engineering

COMmitTeE MembeR: Amelia Greig, Ph.D.

Assistant Professor of Aerospace Engineering

COMMitTeE MEMBER: Thomas Gutierrez, Ph.D.

Professor of Physics

COMMitTeE MEMBER: Insoo Jun, Ph.D.

Group Supervisor/Principal Scientist at NASA/JPL 


\begin{abstract}
Comparative Analysis of Electrodynamic Toroidal Radiation Shielding Configurations
\end{abstract}

Max Rosenberg

Beyond the protective confines of Earth's atmosphere and magnetosphere, spacecraft are subject to constant bombardment by high-energy charged particles originating from our Sun in the form of Solar Particle Events (SPEs), and from outside the solar system in the form of Galactic Cosmic Rays (GCRs). The harm these particles do can be reduced or mitigated outright through radiation shielding. Because protons and other charged particles comprise most of these radiation particles, strong magnetic fields could be generated around spacecraft to deflect incoming charged radiation particles. This thesis investigates the performance of specific configurations of toroidal superconducting solenoids to generate magnetic fields that deflect incoming energetic protons via the Lorentz force. Bulk material shielding configurations using various thicknesses of liquid water are similarly investigated, as are combination shielding configurations combining the best-performing toroidal shielding configurations with a small bulk material shield surrounding the spacecraft.

The water shielding configurations tested included shields of uniform thicknesses from $1 \mathrm{~cm}$ to $10 \mathrm{~cm}$ surrounding an Apollo CSM-sized cylindrical candidate spacecraft. Water shielding was found to be very effective at reducing the SPE dose, from a $86 \%$ reduction at $1 \mathrm{~cm}$ of water to a $94 \%$ reduction at $10 \mathrm{~cm}$. However water shielding was found to be minimally effective against the much higher energy Galactic Cosmic Ray protons, with no dose reduction at $1 \mathrm{~cm}$ and a paltry $1 \%$ reduction at $10 \mathrm{~cm}$.

The toroidal shielding geometric configurations tested consisted of either 5 or 10 primary toroidal shields surrounding the candidate spacecraft, as was the addition of 
smaller nested toroidal shields inside the primary toroids and of toroids on the spacecraft's endcaps. The magnetic field strengths tested were 1.7 Tesla, 8.5 Tesla, and 17 Tesla. The best geometric configurations of electrodynamic shielding consisted of 5 primary toroidal shields, 5 total nested shields placed inside the primary toroids, and 2 total shields on the spacecraft's endcaps. The second best geometric configuration consisted of 10 primary toroidal shields plus two total endcap shields. These configurations at 1.7 Tesla reduced the SPE dose by $87 \%$ and $87 \%$, and reduced the GCR dose by $11 \%$ and $10 \%$. At 17 Tesla, these configurations both reduced the SPE dose by $90 \%$, and reduced the GCR dose by $76 \%$ and $61 \%$. Combining these two configurations with a $1 \mathrm{~cm}$-thick shield of water improved performance against SPE protons to $95 \%$ and $93 \%$ at 1.7 Tesla, and a $97 \%$ and $96 \%$ reduction at 17 Tesla. GCR dose reductions decreased slightly.

Passive material shielding was found capable of providing substantial protection against SPE protons, but was minimally effective against GCR protons without very thick shielding. Electrodynamic shielding, at magnetic field strengths of 1.7 Tesla, was found to be similarly effective against SPE protons, and marginally more effective against GCR protons. Combining the best toroidal shielding configurations, at magnetic field strengths of 1.7 Tesla, with water shielding yielded high protection against SPE protons, but still marginal protection against GCR protons. Increasing the magnetic field strength to 17 Tesla was found to provide very high protection against SPE protons, and to significantly reduce the radiation dose from GCR protons. Of all shielding configurations tested, only those electrodynamic configurations with magnetic fields of 17 Tesla were able to reduce the GCR dose by more than half. 


\section{ACKNOWLEDGMENTS}

Thank you first and foremost to Dr. Kira Abercromby: for many years of exceptional teaching, unrivaled passion about space exploration, and the unending barrage of increasingly difficult homework assignments that have chiseled my engineering and coding capabilities into what they are today.

To the members of my thesis committee Dr. Amelia Greig, Dr. Thomas Gutierrez, and Dr. Insoo Jun: for helping me tackle the immense challenge of reducing down this niche yet complex area of particle physics into a solvable problem, and for demanding the very best from this thesis project and paper.

To my family and friends: for supporting me and believing in me no matter what.

To all musicians past and present: for writing and playing the music to which this thesis project was developed and written, and for showing that thinking outside the box can be a source of progress and inspiration.

"In the hopes that this work may in some way contribute to their exploration of space, this is dedicated to the crew members of this country' s present and future space programs. If only I could join them in their exciting endeavors." -Buzz Aldrin 


\section{TABLE OF CONTENTS}

Page

LIST OF TABLES .................... ix

LIST OF FIGURES $\ldots \ldots \ldots \ldots \ldots \ldots \ldots \ldots \ldots \ldots \ldots \ldots \ldots \ldots \ldots$ CHAPTER

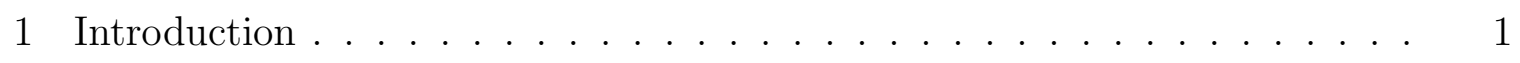

1.1 Statement of Problem . . . . . . . . . . . . . . . . 1

1.2 Proposed Solution . . . . . . . . . . . . . . . . . . 2

1.3 Past Research . . . . . . . . . . . . . . . . . . . . . . . 3

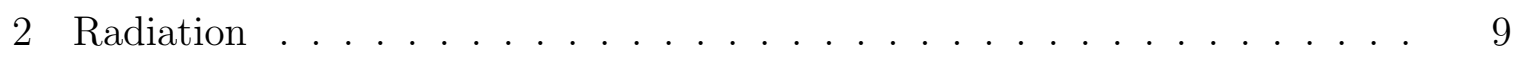

2.1 Properties of Radiation . . . . . . . . . . . . . . . 9

2.2 Effects of Radiation . . . . . . . . . . . . . . . . . . 10

2.3 Radiation Environment . . . . . . . . . . . . . . . . . . 10

$2.3 .1 \quad$ Solar Particles . . . . . . . . . . . . . . . . . . . . . . 11

2.3 .2 Galactic Cosmic Rays . . . . . . . . . . . . . . . 13

3 Radiation Shielding . . . . . . . . . . . . . . . . . . . 14

3.1 Bulk Material Shielding . . . . . . . . . . . . . . . . . 14

3.2 Electrostatic Shielding . . . . . . . . . . . . . . . 16

3.3 Electrodynamic Shielding $\ldots \ldots \ldots \ldots \ldots$

4 Simulation . . . . . . . . . . . . . . . . . 22

4.1 The Particle Simulation Program . . . . . . . . . . . . . 23

4.1 .1 Particle ...................... 26

4.1 .2 Toroid . . . . . . . . . . . . . . . 28

4.1 .3 Payload . . . . . . . . . . . . . . . . . . . 29 


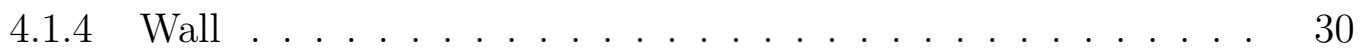

4.2 Analysis and Scoring . . . . . . . . . . . . . . . . 32

4.3 Sample Simulation Run . . . . . . . . . . . . . . . . . . . . . 33

4.4 Testing . . . . . . . . . . . . . . . . . . . . . 41

4.4 .1 Geometric Testing . . . . . . . . . . . . . . . . . 41

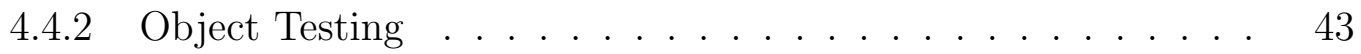

4.4 .3 Statistical Variance Testing . . . . . . . . . . . . . . 45

5 Results. . . . . . . . . . . . . . . . . . . . 47

5.1 Overview . . . . . . . . . . . . . . . . . . 47

5.2 Particle Deflection Performance . . . . . . . . . . . . . 48

5.2 .1 Water Shielding . . . . . . . . . . . . . . 48

$5.2 .2 \quad$ Toroidal Shielding . . . . . . . . . . . . . . . 50

5.2 .3 Combined Water and Toroidal Shielding . . . . . . . . 58

5.3 Radiation Dose Reduction Effectiveness . . . . . . . . . . . . 60

5.3 .1 Water Shielding . . . . . . . . . . . . . . . . 61

5.3.2 Toroidal Shielding and Combined Shielding . . . . . . . . . 63

5.4 Comparison to Past Research _. . . . . . . . . . . . . 67

5.5 Results Summary . . . . . . . . . . . . . . . . . . . . . . . 72

6 Conclusion . . . . . . . . . . . . . . . . . . . 74

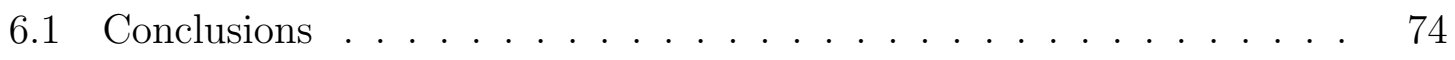

6.2 Future Work . . . . . . . . . . . . . . . . . 77 


\section{LIST OF TABLES}

Table

2.1 Example career Effective dose limits for 1-year missions for a $3 \%$ REID[3] . . . . . . . . . . . . . . . . . 11

4.1 Energy absorption vs. particle energy . . . . . . . . . . . 38

4.2 Energy absorption proportion vs. particle energy . . . . . . . . 39

4.3 SPE and GCR differential flux . . . . . . . . . . . . . . 39

4.4 Radiation dose absorption . . . . . . . . . . . . . 40

4.5 Shielding effectiveness scores . . . . . . . . . . . . . . 41

4.6 Statistical variance test . . . . . . . . . . . . . . 46

5.1 Description of shielding geometric configurations . . . . . . . . . 51

5.2 Water shielding ..................... 63

5.3 Toroidal shielding . . . . . . . . . . . . . . 67

5.4 Shielding effectiveness scores . . . . . . . . . . . . 73 


\section{LIST OF FIGURES}

Figure

Page

1.1 Shielding system surrounding a spacecraft $[8] \ldots \ldots$. . . . . . 5

1.2 Schematic of a toroidal coil layout $[8] \ldots \ldots$. . . . . . . 6

1.3 Various configurations of toroidal shielding $[8] \ldots \ldots$. . . . . . . 7

2.1 Solar Particle Event proton flux [17] . . . . . . . . . . . . . . 12

2.2 Galactic Cosmic Ray flux $[19] \ldots \ldots$. . . . . . . . . . . 13

3.1 Aluminum shielding vs GCRs[20] . . . . . . . . . . . 15

3.2 Electrostatic shielding configurations[21] . . . . . . . . . . . 17

3.3 Cylindrical solenoid magnetic field $[22] \ldots \ldots$. . . . . . . . . . . 19

3.4 Toroidal solenoid particle deflection . . . . . . . . . . . . . 19

3.5 Toroidal shielding efficiency, via JSR $[8] \ldots \ldots$. . . . . . . . . . 20

$4.1 \quad$ Water stopping power[28] . . . . . . . . . . . . . . . 31

$4.2 \quad$ 5-toroid shielding configuration . . . . . . . . . . . . . . . 34

4.3 Particle trajectories at $1 \mathrm{MeV} \ldots \ldots . \ldots 36$

4.4 Particle trajectories at $1 \mathrm{GeV} \ldots \ldots . \ldots 36$

4.5 Statistical variance test . . . . . . . . . . . . . 46

5.1 Unshielded control payload . . . . . . . . . . . . . . 48

5.2 Water shielding . . . . . . . . . . . . . . 50

5.3 Toroidal shielding, $\mathrm{B}=1.7 \mathrm{~T} \ldots \ldots$. . . . . . . . 52

5.4 Toroidal shielding, $\mathrm{B}=8.5 \mathrm{~T} \ldots \ldots . \ldots 54$

5.5 Toroidal shielding, B $=17 \mathrm{~T} \ldots \ldots \ldots$. . . . . . . . . 54 
$5.6 \quad$ Configuration C Rendering . . . . . . . . . . . . . . 55

$5.7 \quad$ Configuration $\mathrm{C} \ldots \ldots \ldots \ldots \ldots \ldots \ldots$

$5.8 \quad$ Configuration E Rendering . . . . . . . . . . . . . . . . . . . 57

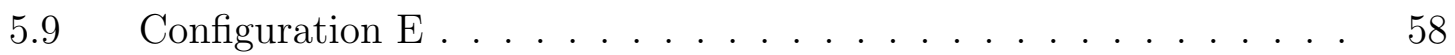

5.10 Configuration $\mathrm{C}^{*} \ldots \ldots \ldots \ldots \ldots \ldots \ldots \ldots$

5.11 Configuration $\mathrm{E}^{*} \ldots \ldots \ldots \ldots \ldots \ldots \ldots$

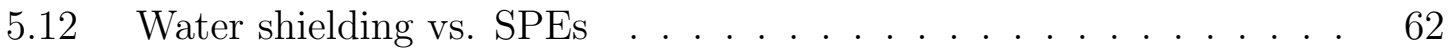

5.13 Water shielding vs. GCRs . . . . . . . . . . . . . 62

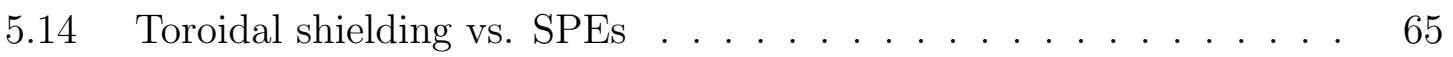

5.15 Toroidal shielding vs. GCRs . . . . . . . . . . . . 66

5.16 Water shielding vs. SPEs, via Gehrke[6] . . . . . . . . . 69

5.17 Water shielding vs. SPEs _. . . . . . . . . . . . . . 69

5.18 Water shielding vs. GCRs, via Gehrke[6] . . . . . . . . 70

5.19 Water shielding vs. GCRs . . . . . . . . . . . . 70

5.20 Configuration D . . . . . . . . . . . . . . . . . 72 
Chapter 1

INTRODUCTION

Humankind's journey to the stars has, up to this point, been limited to the confines of the Earth-Moon system. Where science fiction authors have painted glorious pictures of a society that has broken free from the primitive trappings of scarcity, war, and has embarked on a journey through the cosmos, science fact has placed many hurdles to overcome if that ideal is ever to be reached. Aerospace engineering has, for the past century, been slowly but surely developing solutions to the problems that lie in the way of crewed exploration and habitation of deep space.

\subsection{Statement of Problem}

A spacecraft embarking on a journey past the protective shielding of Earth's atmosphere and magnetosphere will be subjected to high doses of ionizing radiation from the Sun and sources outside our solar system. This ionizing radiation is, at high enough doses, harmful to humans, animals, and even electronics. High enough cumulative exposure to radiation can result in tissue damage and increased cancer risk in humans and animals, and both steady state and transient errors in electronics.

NASA limits astronaut exposure to radiation based on accumulated dose over career to limit the probability of Radiation Induced Exposure Death (REID) to 3\%. These dose levels range from $470 \mathrm{mSv}$ to $1470 \mathrm{mSv}$. Over the course of a 6-month mission on the International Space Station in Low Earth Orbit, astronauts are subjected to $160 \mathrm{mSv}$ of radiation, on average[1]. However past Low Earth Orbit all traces of the protection provided by Earth's atmosphere and magnetic field are completely gone, 
and the interplanetary radiation environment begins. A 6-month trip to Mars in this environment results in $300 \mathrm{mSv}$ of accumulated radiation[2]. Factor in the time spent on Mars and the return trip, and the grand total for a 3-year Mars missions is approximately $1,200 \mathrm{mSv}[1]$. At that level, the only NASA astronauts eligible to be on such a mission, without surpassing NASA's career dose limits, would be males greater than 50 years old with no previous spaceflight experience[3]. This would be their first and last mission.

Changing the solar system's radiation environment to reduce the dose imparted on astronauts is unfeasible. However, the amount of radiation energy that actually is absorbed by astronauts can be reduced through shielding.

\subsection{Proposed Solution}

Radiation shielding can absorb or deflect a portion of incoming radiation energy, reducing the radiation dose experienced by the astronauts, electronics, or other payloads behind it. As radiation particles pass through material, they deposit some of their kinetic energy into that material. This is the mechanism through which ionizing radiation harms biological and electronic payloads, but it is also the mechanism through which bulk material shielding can protect payloads from radiation. By enclosing payloads in a protective shell of dense material such as aluminum or water, all incoming radiation will have to pass through that shell, and in doing so will lose part or all of its kinetic energy, which in turn reduces the amount of harmful radiation energy absorbed by the payloads. This has been the de-facto method of radiation shielding employed for all of space travel, where the spacecraft hull which serves as a pressure vessel also absorbs part of the incoming radiation[1]. To achieve higher levels of radiation protection, more material may be added. However spacecraft are 
often mass constrained; fuel costs increase exponentially with spacecraft mass, and any mass on a crewed spacecraft is already at a very high premium due to other life support and habitation systems. Instead of using mass to absorb radiation, an electric or magnetic field may instead be used to deflect charged radiation particles.

Radiation deflection shielding, so-called because it aims to deflect radiation particles rather than absorb them, offers the possibility of reducing radiation doses experienced by a payload to a greater degree than bulk material shielding of the same mass could. Electrostatic deflection shielding seeks to deflect and slow incoming radiation particles via the generation of a large electric field around the spacecraft. Electrodynamic deflection shielding seeks to deflect incoming radiation particles by the generation of a large magnetic magnetic field. While electrodynamic shielding is the most complex of the three classes of radiation shielding solutions proposed above, using superconductive material to generate high-strength magnetic fields introduces the possibility of very high shielding effectiveness for minimal mass, power draw, and safety concerns. The goal of this thesis is to examine how effective electrodynamic shielding can be compared to bulk material shielding and to examine in what scenarios one might be preferable over the other.

\subsection{Past Research}

The dangers of ionizing radiation have been known since the first decade of the 20th century. Scientists and physicians experienced erythema, swelling, pain, and other tissue damage following prolonged exposure to radioactive elements, and several x-ray machine operators suffered fatal radiation damage. Despite the evidence of these dangers, the recommendations of the British X-ray and Radium Protection Committee were not accepted internationally until two decades later, in 1928[4]. The launch of 
the era of human space exploration in the middle of the 20th century brought with it the many technical problems that must be solved to sustain human life in spacecraft beyond the confines of Earth's atmosphere. Key among these problems was that of the space radiation environment. Beyond the protective confines of Earth's atmosphere and magnetic field, particles originating from the sun and from beyond the solar system delivers substantial doses of harmful ionizing radiation. As the understanding of the space radiation environment developed, so too did the understanding of how to protect against space radiation. Protective materials had now been used for several decades to protect those who worked with radioactive materials. This concept of passive absorption of radiation energy through bulk material could be scaled up and used to protect the crew of spacecraft - universally provided through preexisting spacecraft structural components such as the aluminum hull or storage space, which would absorb some of the radiation energy before it reached the crew inside[5].

Passive radiation shielding has been the only method of radiation protection used in space so far. While traditionally taking advantage of a spacecraft's hull and other structural components to provide modest radiation protection, current developments in the field of passive radiation shielding include examining the use of other mission components, such as food and water storage, to create a radiation shelter. A 2018 master's thesis by Nathan Gehrke at California Polytechnic State University, San Luis Obispo, examined the use of water as a radiation shielding material on crewed space missions beyond Earth orbit[6]. Water is a highly effective radiation absorber and substantial quantities of it are necessary on all crewed space missions. The thesis found water to provide substantial radiation dose reduction from Solar Particle Events, and marginal protection against Galactic Cosmic Rays. The thesis determined a 10-cm thick shield of water would reduce the radiation dose absorbed from the October, 1989 Solar Particle Event by almost 50\%, and would reduce the radiation dose experienced from Galactic Cosmic Rays by approximately 10\%. The 
dose reductions were calculated specifically for the human body, and were made using the On-Line Tools for the Assessment of Radiation in Space (OLTARIS) program, provided by NASA[6].

Active methods of radiation shielding, seeking to repel or deflect incoming charged radiation particles via electromagnetic and electrodynamic forces, have been proposed and mathematically examined since the 1960s. A 1971 report from the Institute of Biomedical Problems in the USSR[7] examined the use of superconducting toroidal solenoids to generate strong magnetic fields that could repel incoming energetic particles, with a system similar to the one illustrated in Fig. 1.1. The authors of the report found that toroidal solenoids with magnetic field strengths of at least 3 Tesla were able to provide substantial protection against radiation particles with energies up to $100 \mathrm{MeV}$ and in doing so would reduce the mass of material shielding required to protect the crew of the spacecraft on a long-duration spaceflight. The proceedings recorded in the report also discuss the power required to cool the superconductors of such a system, which is approximately $30 \mathrm{~kW}$ for the proposed design that includes toroidal solenoids with primary radii of several meters[7].

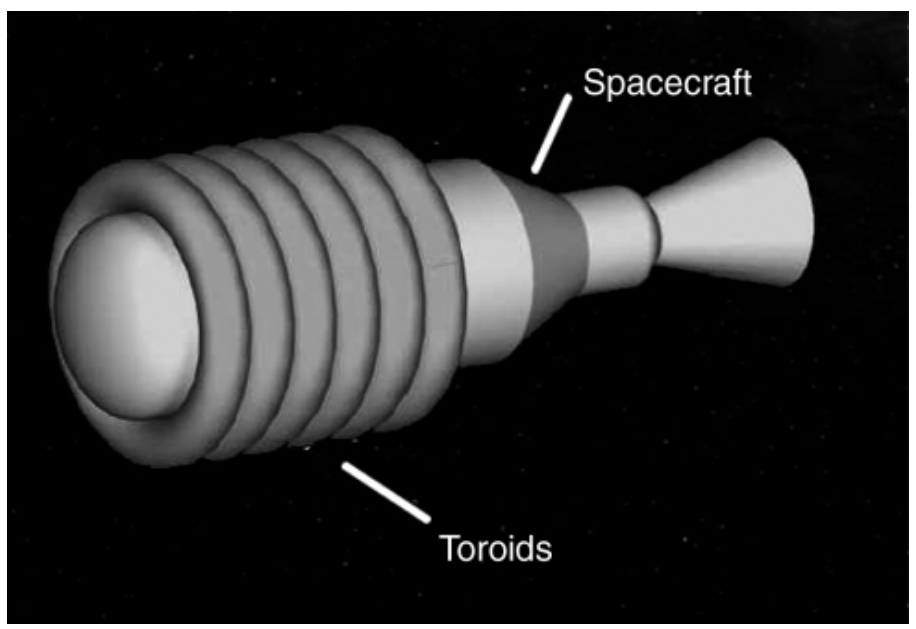

Figure 1.1: Shielding system surrounding a spacecraft [8] 
A 2005 NASA technical memorandum[9] investigated the uses, advantages, disadvantages, and viability of many proposed methods of radiation shielding. Of many advanced radiation shielding concepts reviewed, the authors of this technical memo rated the generation of magnetic fields with with local strong magnets a poor solution. While they calculated it would reduce the radiation dose from GCRs substantially, they found the mass of the required coils would exceed that of passive shielding $[9]$. Furthermore, if superconductive magnetic coils were used, a quench would release a dangerous amount of energy. A quench is when a superconductor exits its superconductive state and enters a normal, resistive state[9]. The most likely cause of superconductor quench in spacecraft operation would be the temperature of the superconductor rising above its critical temperature, at which point the current inside it would be passing through resistant material and heat rapidly due to Joule heating.

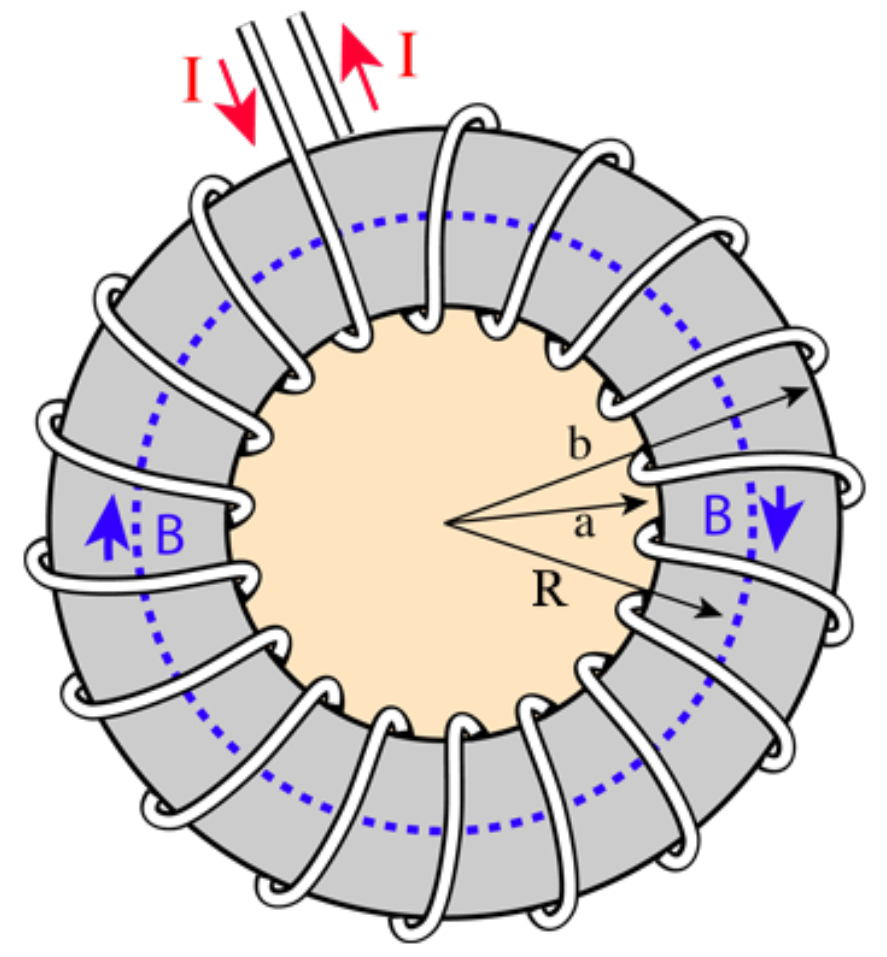

Figure 1.2: Schematic of a toroidal coil layout [8] 
A 2009 article in the Journal of Spacecraft and Rockets[8] specifically examined the performance and feasibility of an inflatable lightweight spacecraft radiation shielding system using superconducting magnet technology. The examined system uses a set of lightweight toroid-shaped coils surrounding a spacecraft to create strong, contained magnetic fields, as shown in Fig. 1.2 and with the configurations shown in Fig. 1.3. Using Monte Carlo analysis, the authors found that the proposed system could deflect more than $90 \%$ of incident particles with $1 \mathrm{GeV}$ of kinetic energy using a 5-T toroidshaped shield. A candidate spacecraft and shield system design is shown in Fig. 1.1. To reduce the mass of structural support for the coils, which was given as a major disadvantage by Adams et. al.[9], the authors propose using lightweight, collapsible structures to support the coils and would be inflated using magnetic pressure. The authors further investigate the uses of such a shielding system as an auxiliary power source, which increases with magnetic field strength and total volume of the coil structure.

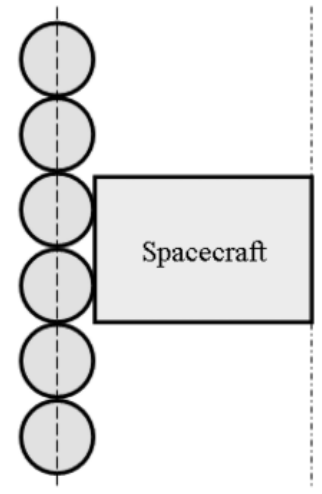

a)

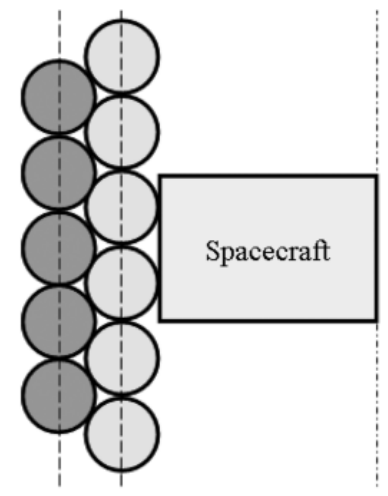

b)

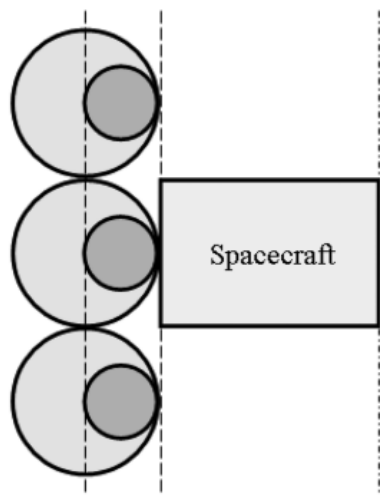

c)

Figure 1.3: Various configurations of toroidal shielding[8] 
Past research in the field of radiation shielding shows substantial research into both passive material shielding and electrodynamic shielding, yet does not do a comparative examination of the relative strengths and weaknesses of these classes of shielding. The primary goal of this thesis is to comparatively evaluate the effectiveness of both passive material and electrodynamic shielding, including performance across the energy spectrum and dose reduction capabilities in both the SPE and GCR radiation environments. Previous research into electrodynamic toroidal shielding does perform some examination of which toroidal shielding configurations provide the best protection against radiation. Secondary goals of this thesis include the comparison of different configurations of toroidal shields, and examining the effectiveness of shielding systems that combine both passive material and electrodynamic shielding. 
Chapter 2

\section{RADIATION}

\subsection{Properties of Radiation}

Radiation describes a wide variety of energetic particles that interact with matter. Radiation can be categorized into particle radiation, and electromagnetic radiation. Particle radiation can be further categorized into protons, neutrons, electrons, and various combinations of those particles. Electromagnetic radiation consists of photons[10]. What distinguishes radiation particles from non-radiation particles is their energy - specifically kinetic energy. The higher the speed of particle radiation, the higher its energy. Electromagnetic radiation, on the other hand, consists of massless photons and therefore its energy is dependent on electromagnetic frequency, rather than its speed, which remains constant at the speed of light.

Radiation can be categorized into ionizing and non-ionizing radiation. Non-ionizing radiation consists of particles that can pass through or be absorbed by matter without depositing enough energy to remove electrons from (ionize) that matter. Ionizing radiation consists of radiation particles with sufficiently high energy to ionize or even break the atomic bonds of matter it passes though. Generally, radiation with energy greater than $10 \mathrm{eV}$ is considered ionizing. For comparison, this is equivalent to a speed of $43.8 \mathrm{~km} / \mathrm{s}$ in protons or neutrons, $1875 \mathrm{~km} / \mathrm{s}$ in electrons, or a wavelength of $124 \mathrm{~nm}$ (in the ultraviolet range) in photons[11]. 


\subsection{Effects of Radiation}

Because ionizing atoms or molecules significantly affects how they behave in chemical interactions, high doses of ionizing radiation are dangerous to biological organisms and electronic systems. In electronics, single energetic particles can cause data corruption, noise in images, system shutdowns, and circuit damage. High accumulated doses can cause degradation of micro-electronics, optical components, and solar cells[12]. In humans, high doses of radiation may cause radiation sickness, increase the risk of cancer, and cause fetal brain damage when pregnant women are exposed[13]. Space agencies such as NASA and ESA impose radiation exposure limits on their astronauts to reduce the probable damage to each astronaut's health and life from space radiation exposure. The career radiation limits are shown in Table 2.1 and are calculated assuming a maximum 3\% permissible probability for Radiation Exposure Induced Death (REID) value. The dose limits increase with the age at which a given astronaut starts their career simply due to older astronauts having fewer years left in their life

over which the radiation exposure may cause health issues. Additionally, the dose limit for female astronauts is smaller to limit the possibility for radiation exposure to cause birth defects to any future children those astronauts might have.

\subsection{Radiation Environment}

The radiation environment describes the abundance of the various species of radiation particles across the energy spectrum at any point in space and at any time. In Earth orbit, the radiation environment primarily consists of solar wind and Solar Particle Events (SPEs), Galactic Cosmic Rays (GCRs), and particles in the Van Allen radiation belts. 
Table 2.1: Example career Effective dose limits for 1-year missions for a $3 \%$ REID $[3]$

\author{
$\mathrm{E}(\mathrm{mSv})$ for a $3 \%$ REID
}

$\begin{array}{lcc}\text { Age at Exposure, y } & \text { Males } & \text { Females } \\ 30 & 620 & 470 \\ 35 & 720 & 550 \\ 40 & 800 & 620 \\ 45 & 950 & 750 \\ 50 & 1150 & 920 \\ 55 & 1470 & 1120\end{array}$

\title{
2.3.1 Solar Particles
}

The corona of the Sun is a large collection of plasma extending millions of kilometers from the Sun's surface and reaching temperatures in excess of 1,000,000 K[14]. The high temperature and magnetic field strength of the corona create and accelerate plasma ions out towards space in all directions. This steady stream of particles is known as solar wind, and generally consists of electrons, protons, and alpha particles with kinetic energies between $0.5 \mathrm{keV}$ and $20 \mathrm{keV}[14]$.

The protons that compose the solar wind typically have insufficient energy to penetrate the Earth's magnetic field, but may be energetic enough to reach the Earth's ionosphere in its polar regions during Solar Particle Events[15]. These events typically last on the order of hours, and a crew exposed to the increased particle activity and energy during that time may be subject to radiation dose rates vastly exceeding maximum permissible dose rates for astronauts, and deliver high enough total doses to cause acute radiation syndrome symptoms, or even chronic and fatal issues in extreme cases[16]. 
The proton flux across the particle energy spectrum is shown in Fig. 2.1 for several different SPE proton models. Because Solar Particle Events are ill-suited to advanced prediction and range in size and strength from barely detectable to catastrophic, Fig. 2.1 gives the fluxes that will not likely be exceeded over a given duration. The solid lines represent maximum probable flux values during any events in a 1-year period at a confidence level of $90 \%$, while the dashed lines represent a 7-year period at a confidence level of $95 \%$. Note that the flux values given are in units of differential flux, and must be integrated through a specific energy range to find the total flux of particles within that range. The SPE proton model used in this thesis is shown in Fig. 2.1 by the dashed blue line, and represents 7 -year maximum probably flux values, at a confidence level of $95 \%$, as calculated using the SAPPHIRE model.

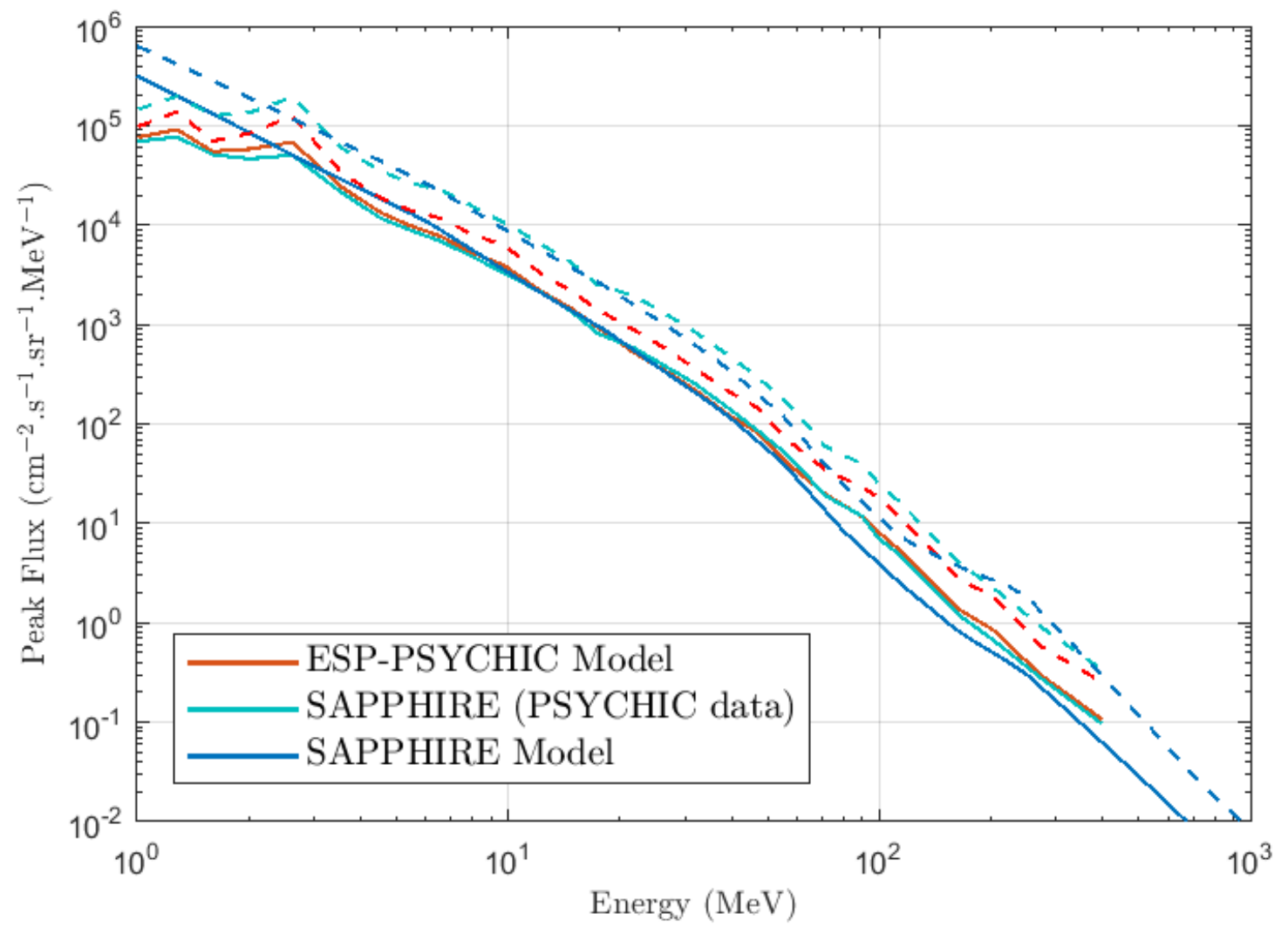

Figure 2.1: Solar Particle Event proton flux [17] 


\subsubsection{Galactic Cosmic Rays}

GCRs are highly energetic protons and atomic nuclei originating from outside the solar system, and typically have kinetic energies measured from $10 \mathrm{MeV}$ to $10 \mathrm{GeV}$. Past the peak flux value, particles of increasing kinetic energy are decreasingly common, as seen in Fig. 2.2. Note that the y-axis units of Fig. 2.2, as with Fig. 2.1 are in units of differential flux and are also divided by particle energy. GCR particle flux is at its highest when solar particle activity is at its lowest, and vice-versa, because the stronger solar magnetic field and solar wind can deflect more incoming GCR particles[18]. The GCR proton model used in this thesis is the ISO-15390 model for GCR proton flux at solar minimum, and is represented in Fig. 2.2 by the red line.

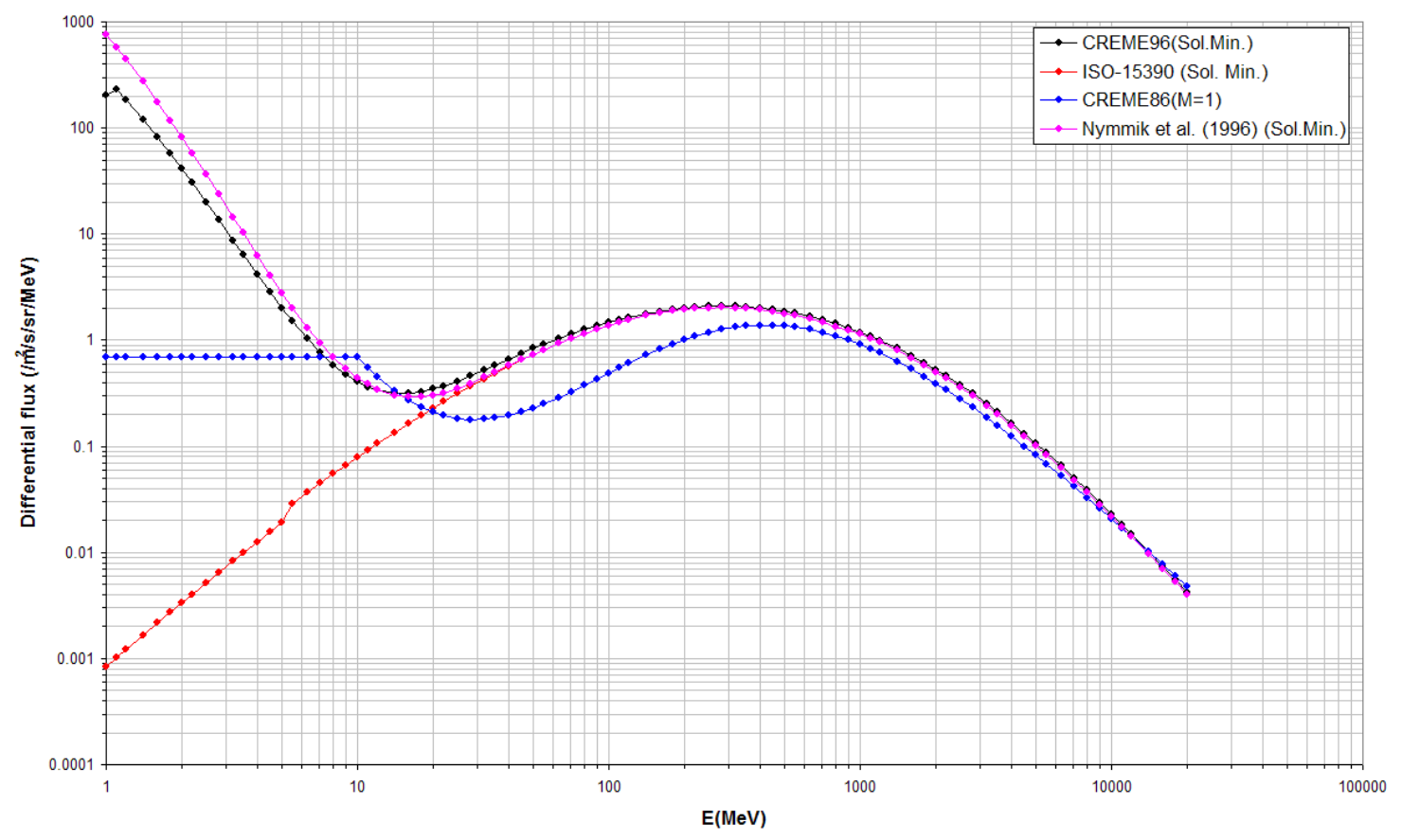

Figure 2.2: Galactic Cosmic Ray flux [19] 
Chapter 3

\section{RADIATION SHIELDING}

Radiation shielding provides the means to protect biological and electronic payloads from the harmful effects of ionizing radiation.

\subsection{Bulk Material Shielding}

Presently, the radiation dose experienced inside a spacecraft is passively reduced by the structural components of the spacecraft. The aluminum walls of inhabited spacecraft are several centimeters thick, and while their primary purpose is to act as a pressure vessel and provide micrometeorid protection, they also provide modest radiation shielding. Shielding thickness is typically given in units of $\frac{g}{\mathrm{~cm}^{2}}$, which is the product of the shield's thickness (in $\mathrm{cm}$ ) and its material density (in $\frac{\mathrm{g}}{\mathrm{cm}^{3}}$ ). Fig. 3.1 shows the effectiveness of aluminum spacecraft walls at providing protection from GCRs; 1 inch of aluminum (at a density of $2.7 \frac{\mathrm{g}}{\mathrm{cm}^{3}}$ ) is equivalent to $6.85 \frac{\mathrm{g}}{\mathrm{cm}^{2}}$ on the x-axis of Fig. 3.1, and results in an approximately $30 \%$ reduction of GCR dose. The GCR environmental model used is CREME, and the GCR transport model used is $\mathrm{UPROP}[20]$. 


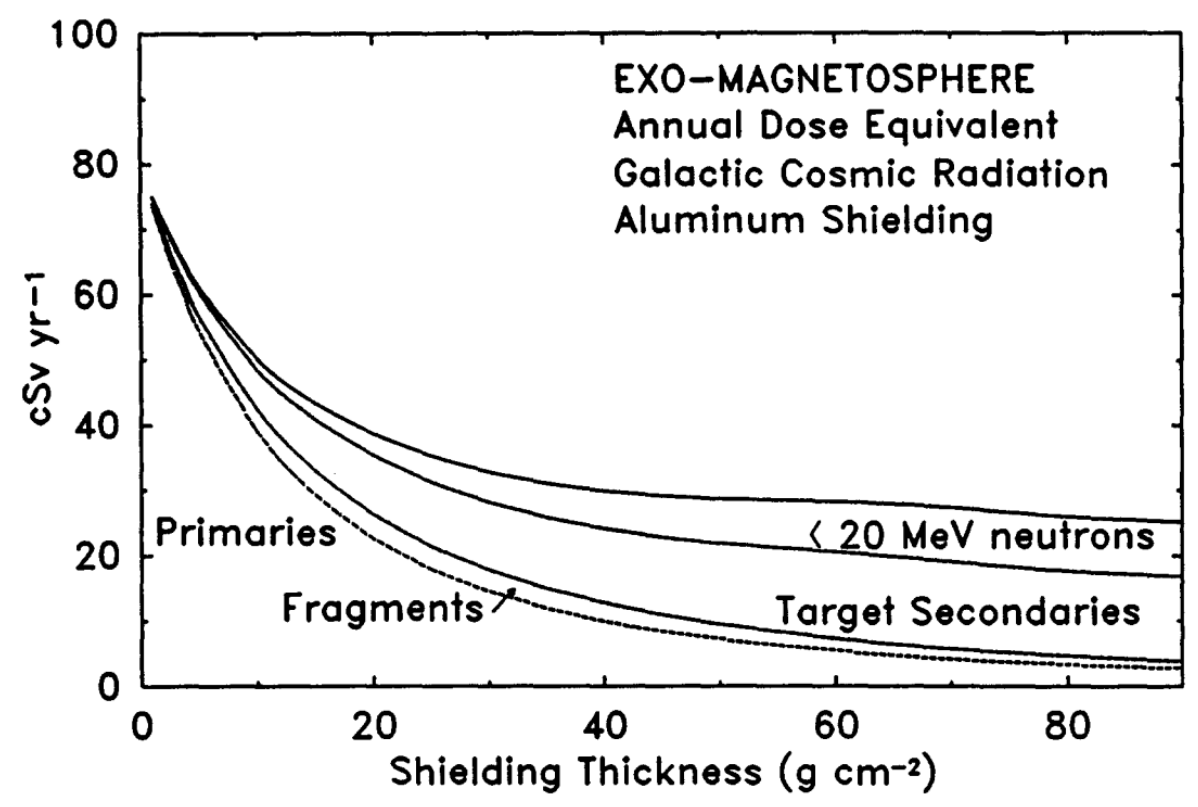

Figure 3.1: Aluminum shielding vs GCRs[20]

Bulk material shielding is advantageous in its simplicity; no power or moving parts are required. Radiation shielding is already provided by structural components in a spacecraft, and spacecraft designers wishing to create areas of higher radiation protection (e.g. for protection during a solar particle event) may place those structural components accordingly.

However, to reach high levels of radiation protection, high amounts of material must be used. To reduce the radiation dose from incoming GCR particles to $25 \%$ of the initial dose, approximately $20 \frac{\mathrm{g}}{\mathrm{cm}^{2}}$ of aluminum shielding must be used, which is equivalent to $7.4 \mathrm{~cm}$. To provide an entire Apollo Command/Service Module (CSM)sized spacecraft with a radiation shield of $7.4 \mathrm{~cm}$-thick aluminum would take $13.3 \mathrm{~m}^{3}$ of aluminum, which has a mass of $35,900 \mathrm{~kg}$. For comparison, the entire launch mass of the CSM was only 14,700 kg. Furthermore, material shielding has the unwanted effect of generating secondary particles. As energetic charged particles pass through 
a material, collisions with the atoms of that material can break the particle apart into smaller, but still very energetic secondary particles that can deliver a radiation dose close to or sometimes even higher than that of the primary particle[20]. However, the addition of dead weight to a spacecraft can be significantly reduced by choosing to use pre-existing components and materials of the spacecraft to create radiation shelters. An example of a a possible configuration that adds minimal weight is using the spacecraft's water supply to surround part of the spacecraft. Water is a very effective radiation absorber[1] and water storage is included on all inhabited spacecraft for life support purposes. By shaping and placing a spacecraft's water storage between all or part the habitable volume and space, radiation doses will be reduced with minimal additional mass costs.

\subsection{Electrostatic Shielding}

Electrostatic radiation shielding is an active shielding method which proposes using high electric potentials may be used to deflect and slow incoming charged particles. These high electric potentials would be on the order of $300,000 \mathrm{kV}$, and would be generated on objects surrounding a spacecraft to provide protection from all directions. However, the high voltages necessitate sufficient distance between the charged objects and the spacecraft to avoid electrostatic breakdown, on the order of hundreds of meters.

Fig. 3.2 shows several possible configurations for such a system. Fig. 3.2a consists of three inner charged toroidal rings and six outer charged spheres designed to repel incoming protons, while Fig. 3.2b uses six positively-charged outer spheres as before to repel protons and also includes six negatively-charged inner spheres to repel solar wind electrons[21]. 
a

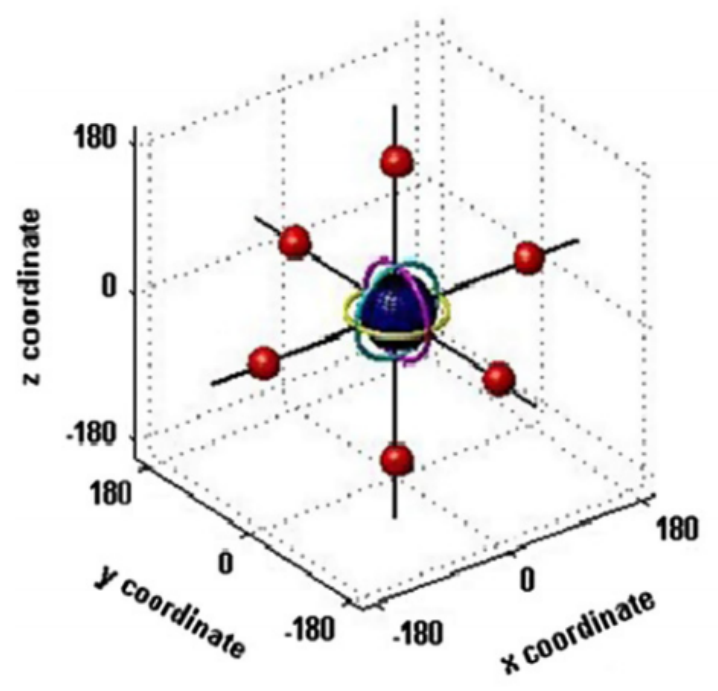

b

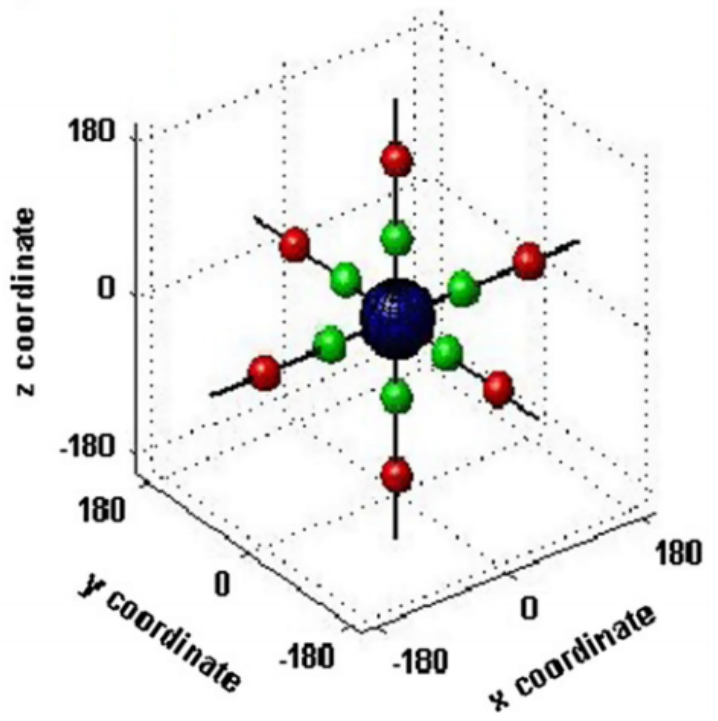

Figure 3.2: Electrostatic shielding configurations[21]

The advantages of electrostatic radiation shielding include significant radiation dose reduction from both solar radiation and GCRs for a relatively low mass cost, and only $\mathrm{mW}$ levels of power are required to maintain the high voltage levels[21]. The primary disadvantage is the aforementioned required separation distance on the order of hundreds of meters between the charged components and the spacecraft to avoid electrostatic breakdown. This necessitates very long and insulated structural components to support such a configuration. Lastly, electrostatic shielding is only able to deflect charged particles. While electrostatic shielding has been calculated to be effective against protons and electrons, it is much less effective against heavier ions such as helium or iron, and is completely ineffective against neutrons. 


\subsection{Electrodynamic Shielding}

Electrodynamic radiation shielding is an active shielding method which, like electrostatic shielding, functions through deflecting incoming charged particles. Rather than generating a strong electric field using high voltages, electrodynamic shielding uses high currents to generate a strong magnetic field to deflect incoming particles using the Lorentz force. The use of a magnetic field, rather than an electric field, means that this type of shielding on its own cannot slow down incoming particles, but merely change their direction. This is because the Lorentz force acts perpendicular to a particle's velocity.

One of the more common families of proposed shielding configurations, and the one that this thesis investigates, is that of a confined magnetic field generated in toroidal solenoids. A solenoid is a helical coil of conductive material through which current is passed to generate a magnetic field. A straight solenoid, as shown in Fig. 3.3, generates an unconfined magnetic field that is strong inside the solenoid, and weak outside. By bending a straight solenoid so that one end meets the other end, a toroid is formed, and the strong interior magnetic field is confined to the toroid's interior. An example of such a system is shown in Fig. 1.1, and a side-view of such a configuration using three toroidal solenoids is shown in Fig. 3.4. In these families of configurations, the use of a toroidal solenoid creates a closed interior volume, meaning the generated magnetic field is confined to the toroidal solenoid's interior volume, and the magnetic field outside of that enclosed volume is negligible. Because of this, such systems can surround or be adjacent to a spacecraft without creating too strong of a magnetic field in the spacecraft's interior. 


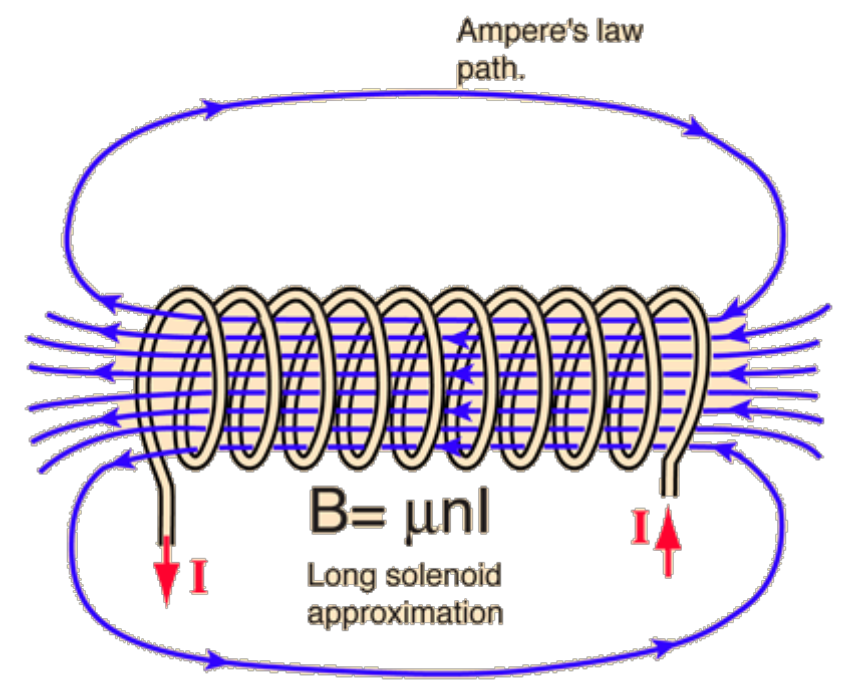

The magnetic

field is

concentrated

into a nearly

uniform field

in the center

of a long

solenoid. The

field outside

is weak and

divergent.

Figure 3.3: Cylindrical solenoid magnetic field[22]

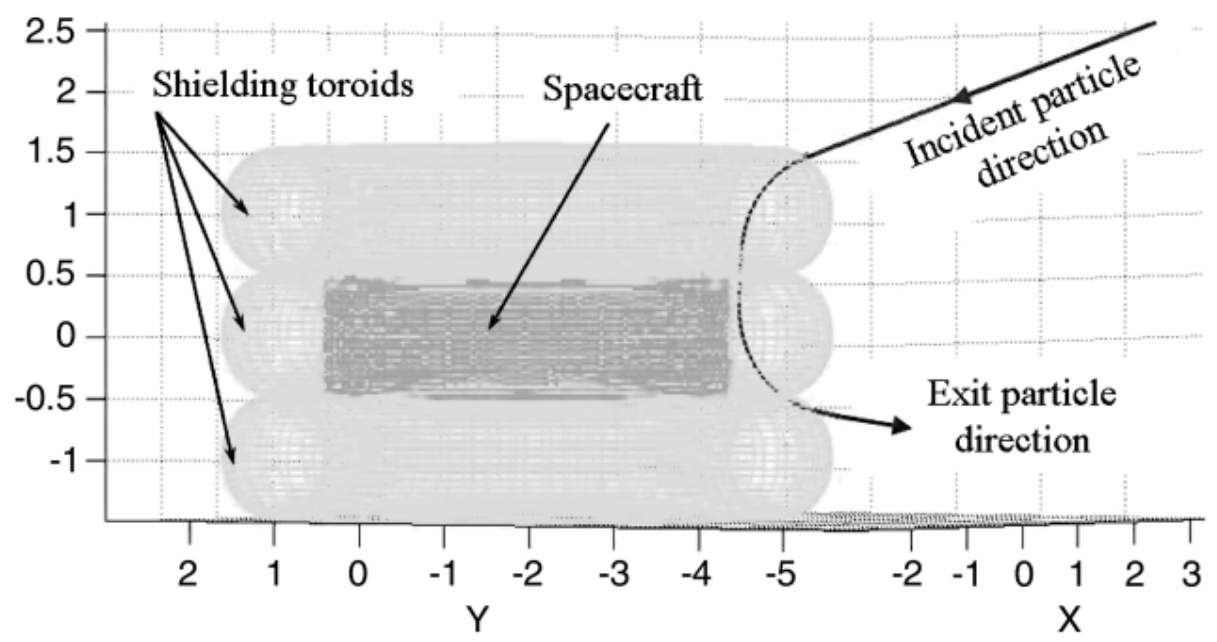

Figure 3.4: Example of a $1 \mathrm{GeV}$ particle deflected away from the spacecraft due to the presence of a 5-T peak magnetic field in each toroid[8]

The effectiveness of electrodynamic shielding increases with magnetic field strength. Fig. 3.5 shows how effectively a proposed electrodynamic shielding system is more effective at deflecting charged particles as the interior magnetic field strength increases. To generate the strong magnetic fields required, which are on the order of 1 to 20 Tesla, very high currents on the order of 10 to $30 \mathrm{kA}$ are needed. To sustain such high 
currents in a normal conductor would require a very large amount of heat dissipation. To vastly reduce both the thermal requirements and power draw, a common proposed solution is to use superconductors.

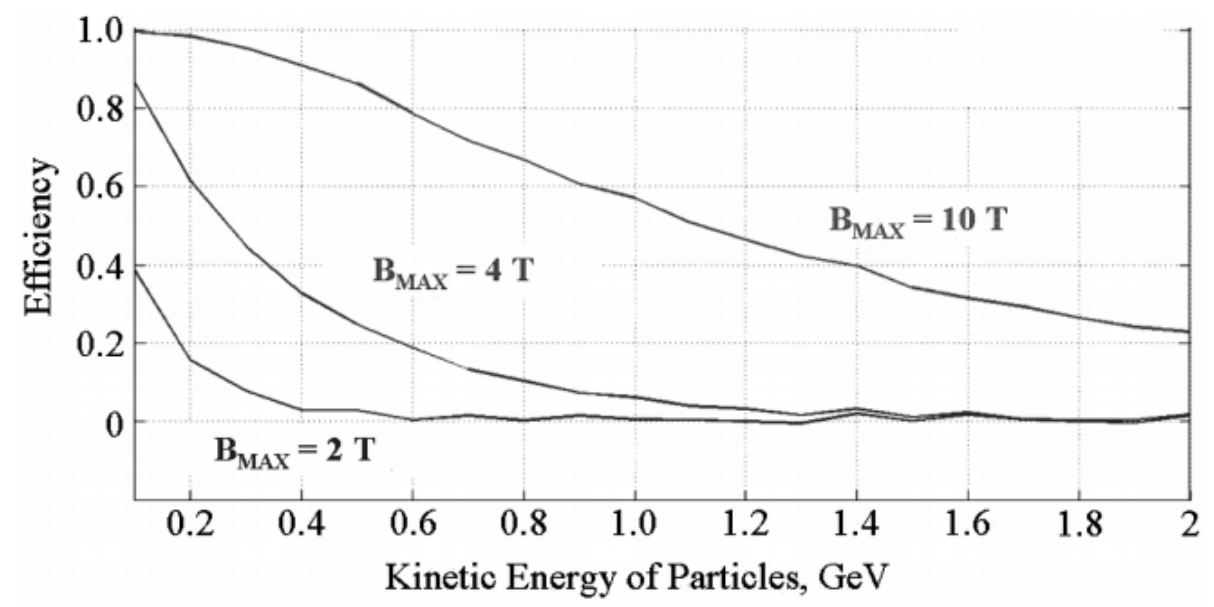

Figure 3.5: Toroidal shielding efficiency, via JSR[8]

Superconductors are materials which have no electrical resistance when below a certain temperature, called the critical temperature. Superconductors can sustain a current without losing any energy to heat dissipation. However superconducting circuits do have a maximum current, which is determined by the critical current density of the superconductive material and the cross-sectional area of the circuit's conductive path. While the superconductive circuit itself draws no power once powered up, some amount of power is required to keep the superconductor below its critical temperature. A popular class of superconductor materials to use are those with critical temperatures above liquid nitrogen - called high-temperature superconductors.

The advantages of an electrodynamic shielding system using toroidal shields composed of high-temperature superconductors are effectiveness against both solar particles and GCRs with moderate space and mass requirements. The primary disadvantage 
is the complexity of the systems required to maintain the high magnetic fields of all the toroids, which include the cryocoolers necessary to keep the superconductors below the critical temperature and the flux pumping needed to generate the high currents. Like with electrostatic shielding, electrodynamic shielding is also ineffective against neutrons, and is less effective against heavy ions which possess high mass but comparatively low electric charge. 
Chapter 4

SIMULATION

The simulation program consists of 2 components: the particle simulation program, written in $\mathrm{C}++$, and the MATLAB script used to analyze the application's outputs. The particle simulation program tests each shielding configuration's effectiveness against many particles across the energetic particle spectrum, and the analysis script applies SPE and GCR proton flux values to calculate radiation doses. Together, these components are used to calculate the dose reduction against both SPE and GCR protons experienced while using various shielding configurations.

The shielding investigated in this thesis includes electrodynamic shielding consisting of toroidal solenoids, passive material shielding consisting of water, and combinations of the two. The particle simulation application characterizes a given shielding configuration's effectiveness in reducing or eliminating the radiation dose imparted on a payload object by radiation particles using these steps:

1. Place many identical particles of a given energy level on random points of a sphere surrounding the payload and shielding.

2. Set the particles' velocities so that they are travelling directly towards the payload object.

3. Propagate each particle forward in time using its equation of motion as shown in Eq. 4.5 until either:

(a) The particle is inside the payload, at which point calculate the radiation energy absorbed by the payload and, if applicable, the wall

(b) The particle has passed by the payload without impact 
4. Repeat steps 1-3 for many different particle energy levels.

The output files are then taken by the MATLAB script to analyze.

\subsection{The Particle Simulation Program}

The simulation program reads the simulation parameters file to generate the objects listed below, with the following user-controlled properties:

1. Particle
(a) Energy levels $(e V)$
(b) Particles per energy level (\#)

2. Toroids
(a) Primary radius $(m)$
(b) Secondary radius $(m)$
(c) Number of turns (\#)
(d) Current $(A)$
(e) Position $(m)$
(f) (Optional) Critical current density $\left(\frac{A}{m^{2}}\right)$

3. Payload (Carbon)
(a) Radius (m)
(b) Height $(m)$
(c) Density $\left(\frac{g}{\mathrm{~cm}^{3}}\right)$

4. Wall (Water)
(a) Radius $(m)$
(b) Height $(m)$ 
(c) Density $\left(\frac{g}{\mathrm{~cm}^{3}}\right)$

The following assumptions governing each object and its interactions with particles:

- The only particles considered are protons. The majority of SPE and GCR particles are protons, and a significant portion of the radiation dose from SPEs and GCRs comes from protons. While other particles such as electrons, neutrons, and heavier ions are significant in the space radiation environments, basic conclusions about shielding performance against other particles can be drawn from shielding performance against protons.

- Special relativity is used. The energetic particles present in the SPE and GCR radiation environments are travelling at a significant percentage of the speed of light.

- Toroids, payloads, and walls are all stationary in a stationary reference frame.

- The particle's total energy is conserved, and the effects of the Lorentz force on the particle's momentum is adjusted accordingly.

- The initial velocity of each particle is calculated relativistically from its given energy.

- Interactions between multiple particles are neglected. The electric fields created by the protons are so small, and the distribution of radiation particles is effectively random enough to neglect electrostatic forces charged particles exert on each other.

- Particles retain their mass and composition throughout the simulation, i.e. secondary particle generation and effects are neglected. Because protons are the radiation particle in use, and water is the shielding material in use in the simula- 
tions, secondary particle generation is already minimized compared to shielding materials such as aluminum.

- Particles travel in a straight line unless acted upon by the Lorentz force. Forces caused by electromagnetism are the dominant forces at the scales involved in this simulation (on the order of meters).

- Particles' paths through matter are straight lines. This is the average case behavior of an energetic particle travelling through a material.

- Each toroid generates zero magnetic field outside its volume. The magnetic field outside a solenoid, and particularly a toroidal solenoid, is negligible compared to the strength of the magnetic field inside.

- The magnitude of each toroid's magnetic field remains constant. The deviations of magnetic field strength are either negligible in value or occur across a negligibly small volume.

- The direction at any point of each toroid's magnetic field inside the toroid is equal to the cross product of the vector from the toroid's origin to the point in question, and the toroid's primary axis of revolution. Directional deviation of the magnetic field vector is negligible.

- Interactions between particles and the materials making up each toroid are neglected. The flux of energetic particles is low enough that the effects on each toroid from the sun and other particles in space will have much more of an effect.

- Interactions between particles and the current or magnetic field levels of each toroid are neglected. The total flux levels of charged energetic particles in space from the SPEs and GCRs is many orders of magnitude less than a single Ampere. Furthermore, the associated magnetic flux pump that would be used in an actual shielding system like this to induce a current in the solenoids is assumed to be keeping the solenoid current at its critical current value. 


\subsubsection{Particle}

The particle's state is stored as a 3 degree-of-freedom state vector as shown in Eq. 4.1 .

$$
\vec{x}=\left[\begin{array}{ll}
\vec{r} & \vec{v}
\end{array}\right]^{T}=\left[\begin{array}{llllll}
r_{x} & r_{y} & r_{z} & v_{x} & v_{y} & v_{z}
\end{array}\right]^{T}
$$

The particle's origin point is chosen as a random point on a sphere of radius $100 \mathrm{~m}$. The particle's target point is also randomly chosen as a point on a sphere with a radius equal to either the cylindrical payload's half-height or its radius: whichever is smaller. The points are chosen from a random spherical distribution through the use of Eq. 4.2, where $R$ is the radius of the sphere, and $x_{0}, x_{1}, x_{2}$, and $x_{3}$ are randomly chosen from a uniform distribution on $(-1,1)$ until the inequality shown in Eq. 4.3 is satisfied[23]. The position of the particle may then be represented by the vector $\left[\begin{array}{lll}x & y & z\end{array}\right]^{T}$.

$$
\begin{gathered}
x=\frac{2\left(x_{1} x_{3}+x_{0} x_{2}\right)}{x_{0}^{2}+x_{1}^{2}+x_{2}^{2}+x_{3}^{2}} R \\
y=\frac{2\left(x_{2} x_{3}-x_{0} x_{1}\right)}{x_{0}^{2}+x_{1}^{2}+x_{2}^{2}+x_{3}^{2}} R \\
z=\frac{x_{0}^{2}+x_{3}^{2}-x_{1}^{2}-x_{2}^{2}}{x_{0}^{2}+x_{1}^{2}+x_{2}^{2}+x_{3}^{2}} R \\
x_{0}^{2}+x_{1}^{2}+x_{2}^{2}+x_{3}^{2}<1
\end{gathered}
$$

The initial speed of the particle is determined from the particle's given kinetic energy, and is calculated taking into account special relativity using Eq. 4.4 where $E$ is the kinetic energy in Joules, and $m_{0}$ is the particle mass mass. 


$$
\begin{aligned}
& v=\beta c \\
& \beta=\sqrt{1-\frac{1}{\gamma^{2}}} \\
& \gamma=\frac{E}{m_{0} c^{2}}+1
\end{aligned}
$$

The particle's state derivative is calculated using its equations of motion as shown in Eq. 4.5, where $F_{L}$ is the Lorentz force acting on the particle and $m$ is taken from the particle's relativistic momentum, which has historically been called "relativistic mass." This differential equation is integrated using an adaptive-step fourth-order Runge-Kutta solver with fifth-order error calculation provided by the Odeint $\mathrm{C}++$ library.

$$
\dot{\vec{x}}=\left[\begin{array}{ll}
\vec{v} & \frac{\overrightarrow{F_{L}}}{m}
\end{array}\right]^{T}
$$

The Lorentz force on the particle may be calculated through Eq. 4.6, where $q$ is the charge of the particle in Coulombs, and $\vec{B}$ is the magnetic field vector.

$$
\overrightarrow{F_{L}}=q(\vec{v} \times \vec{B})
$$

To account for the relativistic momentum of the particle, the value used to calculate the effect of the Lorentz force on the particle in the inertial frame, historically referred to as "relativistic mass," may be calculated using Eq. 4.7, where $m_{0}$ is the particle's mass, $v$ is the particle's speed in the stationary reference frame, and $c$ is the speed of light.

$$
m=\frac{m_{0}}{\sqrt{1-\frac{v^{2}}{c^{2}}}}
$$




\subsubsection{Toroid}

The purpose of the toroid in this hypothetical physical system is to generate a magnetic field strong enough, and in the proper direction, to deflect incoming charged particles via the Lorentz force shown in Eq. 4.6. The simulated toroids are composed of the superconductor bismuth strontium calcium copper oxide (BSCCO). BSCCO was chosen for its relatively high critical temperature of approximately $100 \mathrm{~K}$, and its ability to maintain a conductive path as either a powder-in-tube or as a conductive tape, either of which would be suitable for use in the described toroidal solenoid, provided sufficient cryocooling and structural support were provided[24]. The critical current density of BSCCO is $5 \cdot 10^{5} \frac{A}{\mathrm{~cm}^{2}}$ and its density is $11.4 \frac{\mathrm{g}}{\mathrm{cm}^{3}}[25]$.

The magnetic field vector in Tesla inside a toroidal solenoid is calculated using Eq. 4.8. In the magnetic field magnitude calculation $R$ is the primary radius in meters, $N$ is the number of turns in the solenoid as seen in Fig. 1.2, $I$ is the current passing through the wire in Amperes, and $\mu_{0}$ is the vacuum permeability of free space[26]. The magnetic field direction unit vector at any interior point is calculated as the cross product of $\hat{r}$, the directional unit vector from the toroid's center to the interior point, and $\hat{z}$, the unit vector aligned with the toroid's axis of revolution.

$$
\begin{aligned}
& \vec{B}=B \hat{b} \\
& B=\frac{\mu_{0} N I}{2 \pi R} \\
& \hat{b}(\vec{r})=\hat{r} \times \hat{z}
\end{aligned}
$$

The direction of the magnetic field follows the right-hand-rule and is perpendicular to the flow of electrons through the wires around each turn, as seen in Fig. 1.2. 


\subsubsection{Payload}

The payload is a cylinder of user-defined radius and height, aligned with the global z-axis. In the simulations run for this thesis, the cylinder has a radius of $2.5 \mathrm{~m}$ and a height of $10 \mathrm{~m}$. The payload material and density used in this thesis are described in the Sample Simulation section. To determine if a radiation particle passes through the payload, ray tracing methods are used, as shown below.

The quadratic equation $a t^{2}+b t+c=0$, with coefficients calculated using Eq. 4.9, has roots at the the time(s) of intersection between a ray and an unbounded (uncapped) cylinder. In this equation, $\mathrm{r}$ and $\mathrm{v}$ are the ray's position and velocity, and $\mathrm{R}$ is the cylinder's radius.

$$
\begin{aligned}
& a=v_{x}^{2}+v_{y}^{2} \\
& b=2 r_{x} v_{x}+r_{y} v_{y} \\
& c=r_{x}^{2}+r_{y}^{2}-R^{2} \\
& d=b^{2}-4 a c
\end{aligned}
$$

If $d>0$, as calculated in Eq. 4.9, then the ray has two intersections with the unbounded cylinder, the times of which may be calculated using Eq. 4.10.

$$
\begin{aligned}
& t_{1}=\frac{-b-\sqrt{d}}{2 a} \\
& t_{2}=\frac{-b+\sqrt{d}}{2 a}
\end{aligned}
$$

Because the payload is a bounded cylinder (with endcaps), we must check to see if the intersection points occur within the bounds of the cylinder's endcaps. Because in this simulation, the payload cylinder is necessarily aligned with the global z-axis, 
the first step is to calculate the heights of the ray's intersection with the unbounded cylinder, as shown in Eq. 4.11.

$$
\begin{aligned}
& p_{z_{1}}=r_{z}+v_{z} t_{1} \\
& p_{z_{2}}=r_{z}+v_{z} t_{2}
\end{aligned}
$$

Each of the z-coordinates of the intersection points with the unbounded cylinder, as calculated above in Eq. 4.11, is then evaluated against the geometry of the cylinder to see if the points occur within the confines of the bounded cylinder, or if the actual intersection point occurs at the cylinder's endcap. This problem is solved using Eq. 4.12 , where $p_{z}$ is the z-coordinate of the point in question, $z_{\max }$ and $z_{\min }$ are the z-coordinates of the top and bottom of the cylinder, respectively, and $z$ refers to the z-coordinate of the endcap closest to the ray's intersection with the unbounded cylinder.

$$
\vec{p}= \begin{cases}\vec{r}+t \vec{v}, & \text { if } z_{\max }>p_{z}>z_{\text {min }} \\ \vec{r}+\frac{z-r_{z}}{v_{z}} \vec{v}, & \text { otherwise }\end{cases}
$$

\subsubsection{Wall}

The wall is an optional material shield that may be included in each simulation. Like the toroid, its purpose is to reduce the radiation energy absorbed by the payload. Rather than deflecting incoming particles using the Lorentz force, the wall reduces radiation energy absorbed by slowing down incoming particles, thus reducing their kinetic energy. In general this will reduce the radiation energy absorbed by the payload. However, because the absorption of radiation energy is a complex process, 
and the stopping power of a material is dependent on a particle's energy at any point, it is possible for the introduction of a material shield to actually increase the radiation energy absorbed by a payload, even though the particle would enter the payload with lower energy. The stopping power of a material on an energetic particle tends to increase as particle energy decreases, because the size of the particle's interaction cross-section also increases with decreasing energy[27]. This phenomenon is illustrated in Fig 4.1.

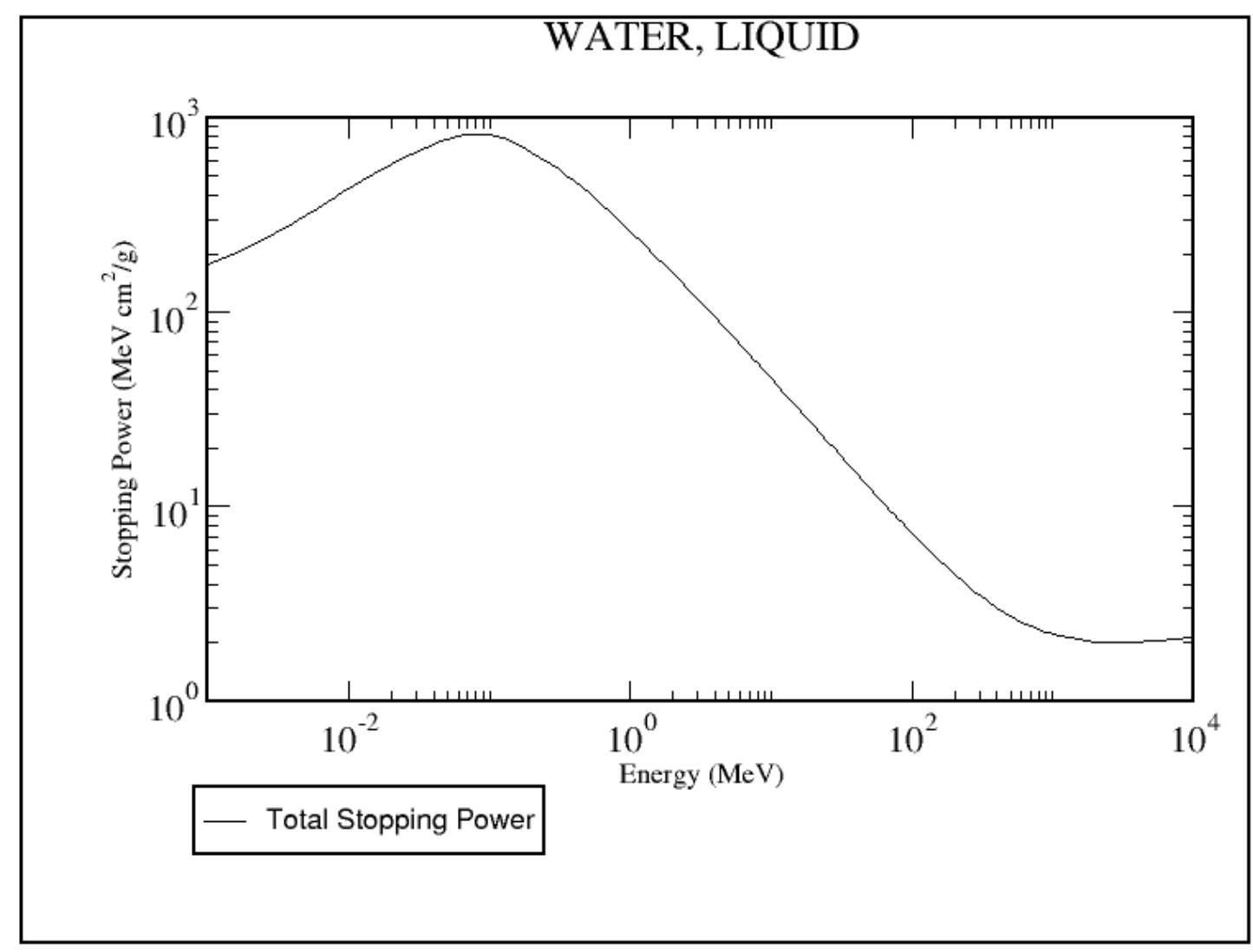

Figure 4.1: Water stopping power[28]

In the simulations run for this thesis, those using a wall as a material shield for the payload used a wall of water, as water both effectively absorbs radiation and is necessary for crewed space travel. 


\subsection{Analysis and Scoring}

To enable comparisons between the effectiveness of different shielding configurations, each configuration is scored. The score is the dose reduction factor that may be used to calculate the radiation dose experienced by the shielded payload from SPEs or GCRs, as compared against an unshielded control payload. When a particle intersects the payload or the protective wall, the energy absorbed by the object is calculated using simple Linear Energy Transfer as shown in Eq. 4.13. To calculate the energy absorbed, a definite integral is done over the intersection path length $L$, where the

stopping power $L E T$ in $M e V \frac{\mathrm{cm}^{2}}{g}$ as a function of energy is evaluated at every step and multiplied by the material density $\rho$ in $\frac{g}{\mathrm{~cm}^{3}}$ to get $\frac{d E}{d x}$ in $\frac{\mathrm{MeV}}{\mathrm{cm}}$.

$$
\begin{aligned}
& E_{\text {absorbed }}=E_{i}-E_{f}=E_{i}-\int_{0}^{L} \frac{d E}{d x} d x \\
& \frac{d E}{d x}=L E T \cdot \rho
\end{aligned}
$$

The primary output of the particle simulation is, for every energy level simulated, the percentage of the total particle kinetic energy deposited into the payload, as shown in Eq. 4.14 where $i$ is the index number of the particle, $N$ is the number of particles simulated at that energy level, $E$ is the initial energy of each of the particles, and $E_{\text {absorbed }}$ is the energy absorbed by the payload for particle $i$.

$$
p(E)=\frac{\sum_{i=1}^{N} E_{\text {absorbed }}}{N E}
$$

After evaluating this metric for all the energy levels that had been simulated, the data vector $\bar{p}$ now represents a discretized approximation of a function of energy level that gives the expected proportion of cumulative kinetic energy that would be absorbed by a payload subjected to any number of particles all at a given energy level. 
The absorbed dose may then be calculated by performing an integral as shown in Eq. 4.15, where $s$ is the particle energy level, and $S$ is the domain of $s$ over which simulations were run, $\frac{d F}{d E}(s)$ is the particle differential flux as a function of a given particle energy level, and is taken from Fig. 2.1 for the Solar Particle Event radiation environment, or Fig. 2.2 for the Galactic Cosmic Ray radiation environment..

$$
d=\int_{S} \frac{d F}{d E}(s) s \frac{p}{p^{*}} d s
$$

Eq. 4.15 is discretized and integrated using the trapezoidal rule, and using the data vector forms of $F, s$, and $p$, where $p^{*}$ is $p$ evaluated for a configuration with no shielding.

The score, $R$, of the shielding configuration is then calculated by simply dividing the dose imparted on the unshielded dose, $d^{*}$ by the shielded dose, $d$, as shown in Eq. 4.16 .

$$
R=\frac{d^{*}}{d}
$$

\subsection{Sample Simulation Run}

The simulation process was demonstrated for an Apollo CSM-sized payload protected by 5 electrodynamic toroidal shields, as shown in Fig. 4.2 . The cylindrical payload has a radius of $2.5 \mathrm{~m}$, a height of $10 \mathrm{~m}$, a material composition of amorphous carbon, and a density of $0.2 \frac{\mathrm{g}}{\mathrm{cm}^{3}}$. Amorphous carbon was used as the material to approximate the radiation absorption curves of human tissue. While amorphous carbon has a nominal density of $2.0 \frac{\mathrm{g}}{\mathrm{cm}^{3}}, 10 \%$ of that nominal value was used instead. This decrease in

density to $10 \%$ of the nominal density is meant to represent the the payload as a 
habitable volume composed of $10 \%$ human astronauts and $90 \%$ empty space. This is meant to approximate the habitable volume of actual spacecraft, which is mostly air. Each toroid has a primary radius of 3.5 meters, a secondary radius of 1 meter. The solenoid component of each toroid has 1000 turns and carries a current of $30 \mathrm{kA}$, giving each toroid an internal magnetic field of 1.714 Tesla, using Eq. 4.8.
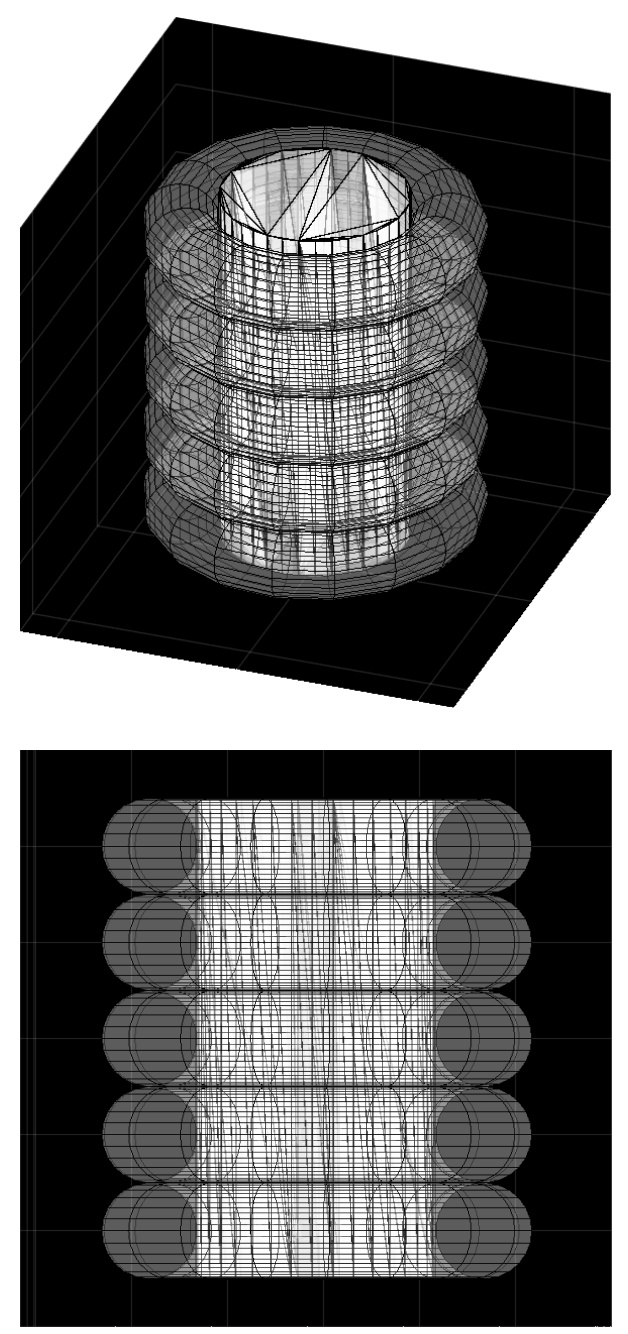

Figure 4.2: 5-toroid shielding configuration 
For this sample simulation, the payload and shielding are then subjected to bombardment by 100 particles of each of the following energy levels:

- $1 \mathrm{MeV}$

- $10 \mathrm{MeV}$

- $100 \mathrm{MeV}$

- $1 \mathrm{GeV}$

- $10 \mathrm{GeV}$

The actual simulation uses 14 different energy levels, rather than 5, and the upper energy limit is extended to $20 \mathrm{GeV}$.

The particle trajectories for $1 \mathrm{MeV}$ and $1 \mathrm{GeV}$ are shown below in Figs. 4.3 and 4.4. Note that to reduce visual clutter, only 10 of the 100 particles per energy level are shown. Green lines show the trajectories of particles that are deflected by the toroids, and thus do not impact the payload. Red lines show the trajectories of particles that are not deflected enough by the toroids, and thus still impact the payload. While there is no indication of the incoming or outgoing direction of each trajectory, there is no practical difference because the force due to a non-changing magnetic field is conservative. 


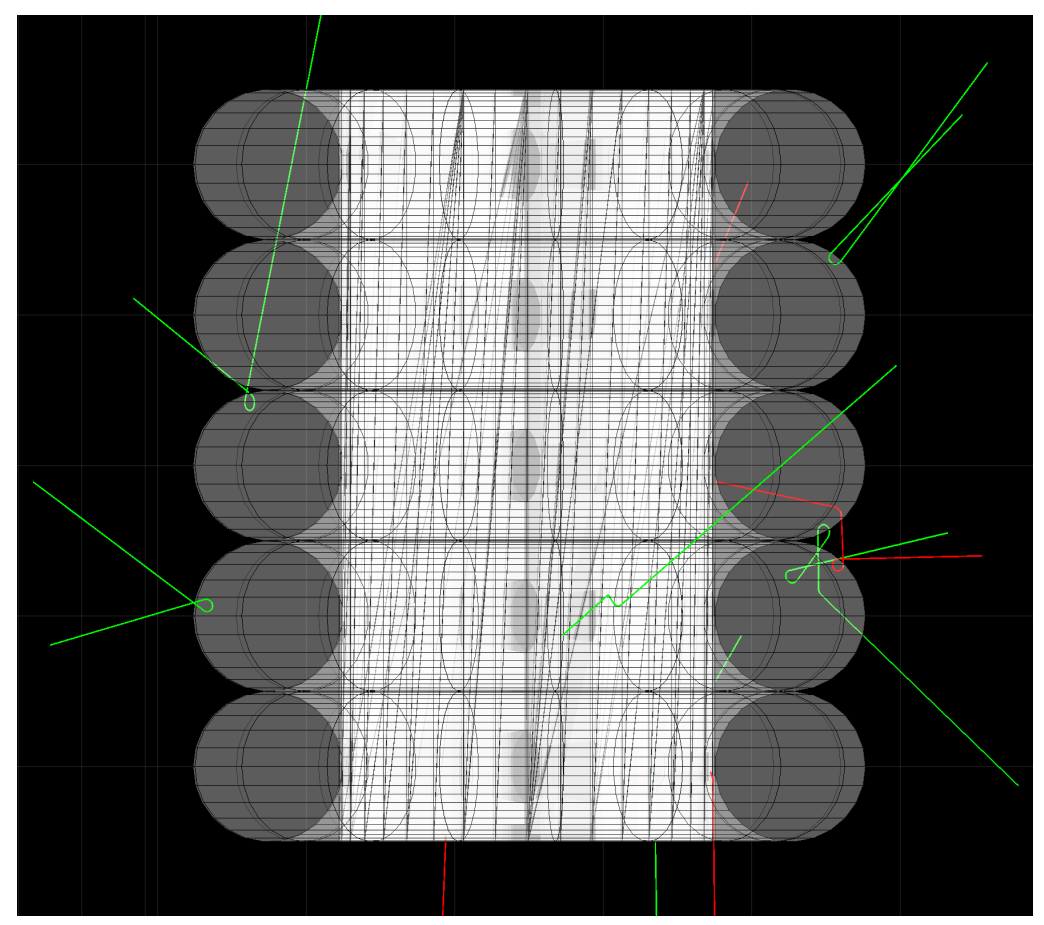

Figure 4.3: Particle trajectories at $1 \mathrm{MeV}$

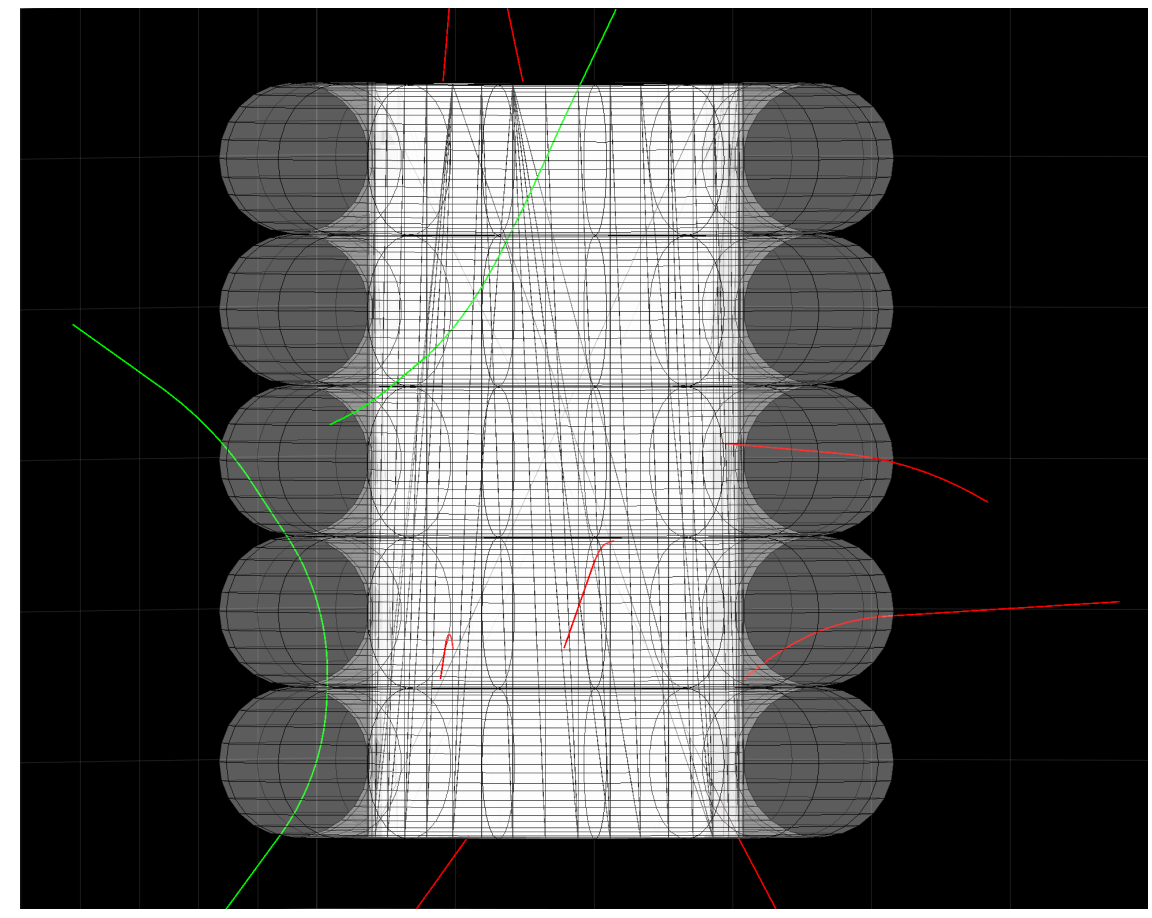

Figure 4.4: Particle trajectories at $1 \mathrm{GeV}$ 
The effect of particle speed on each particle's radius of gyration is easily visible; more energetic particles have larger radii of gyration, and are thus deflected less by the same strength magnetic field. A particle's radius of gyration decreases proportional to the component of its velocity that is perpendicular to the magnetic field the particle is travelling through, as seen in Eq. 4.17[29].

$$
r_{g}=\frac{m v_{\perp}}{|q| B}
$$

For energies below $1 \mathrm{GeV}$, the toroidal shields are able to deflect most incoming particles. Those particles that are not deflected generally enter the payload through the relatively unprotected area between toroids. Higher energy particles tend to only be successfully deflected if the incident angle is already sufficiently high; in these cases, the deflection induced by the magnetic field merely gives the incoming particle enough of a push in the right direction to avoid impact with the payload.

The total energy absorbed by the payload, as a fraction of source particle-energy, is shown in Table 4.1. For example, the second row of data indicates that when subjected to bombardment by 100 particles with $10 \mathrm{MeV}$ of energy, the unshielded control payload absorbs all 100 of those particles ( $1 \mathrm{GeV}$ total energy), and our shielded payload absorbs 27 of those particles (270 MeV total energy). The control payload absorbs the entirety of the energy of all incident particles for the energy levels up to $1 \mathrm{GeV}$. However at the $10 \mathrm{GeV}$ energy level, only $97.77 \%$ of the total kinetic energy is absorbed. The reason for this is that the typical maximum range of a $10 \mathrm{GeV}$ proton through amorphous carbon is on the order of $10 \mathrm{~s}$ of meters, an order of magnitude greater than the range for a $1 \mathrm{GeV}$ proton through the same material. It therefore follows that at energy levels above $1 \mathrm{GeV}$, some protons will travel through the payload without depositing all of their kinetic energy, which is 
exactly what a value of $97.77 \%$ shows to have happened. The data from the energy deposition in the shielded payload corroborates the pattern observed in Figs. 4.3-?? that higher-energy particles are less easily deflected by the shields. The only exception is that moderately less energy is absorbed in the shielded payload from particles of $100 \mathrm{MeV}$ than is absorbed from particlels of $10 \mathrm{MeV}$. While the particles' initial states are randomized, this fluctuation is the result of a physical phenomenon, rather than statistical variance. This is shown and discussed in the Testing section.

\section{Table 4.1: Energy absorption vs. particle energy} \# of particles worth of energy absorbed

$\begin{array}{rccc}\text { Energy } & \text { Number of particles (N) } & \text { Control } & \mathbf{5} \text { Toroids } \\ 1 \mathrm{MeV} & 100 & 100 & 17 \\ 10 \mathrm{MeV} & 100 & 100 & 27 \\ 100 \mathrm{MeV} & 100 & 100 & 22 \\ 1 \mathrm{GeV} & 100 & 100 & 76 \\ 10 \mathrm{GeV} & 100 & 97.77 & 92.79\end{array}$

Using Eq. 4.14, where the particle energy vector $\vec{E}$ is $[1 e 6,1 e 7,1 e 8,1 e 9,1 e 10] \mathrm{eV}$, the proportions of particle kinetic energy absorbed are calculated from the above data and shown in Table 4.2. The control-adjusted proportion $\frac{p}{p^{*}}$ is also shown in the rightmost column. This control-adjusted proportion is the most important data that comes directly from the particle simulation results, before the radiation environments are considered. Because the control payload absorbed nearly all of the kinetic energy from the incident particles, the control-adjusted proportions are nearly identical to the non-control-adjusted proportions. 
Table 4.2: Energy absorption proportion vs. particle energy Average Proportion of Particle Energy Absorbed

$\begin{array}{rccc}\text { Energy } & \text { Control }\left(p^{*}\right) & \mathbf{5} \text { Toroids }(p) & \mathbf{5} \text { Toroids, adjusted for control }\left(\frac{p}{p^{*}}\right) \\ 1 \mathrm{MeV} & 1 & .17 & .17 \\ 10 \mathrm{MeV} & 1 & .27 & .27 \\ 100 \mathrm{MeV} & 1 & .22 & .22 \\ 1 \mathrm{GeV} & 1 & .76 & .76 \\ 10 \mathrm{GeV} & .98 & .93 & .95\end{array}$

To calculate the doses, the particle differential flux values are first needed for each of the energy levels simulated. The differential flux values are shown in Table 4.3 for both SPEs and GCRs. The SPE differential flux values are those of the 7-year maximum probable flux as defined by the SAPPHIRE model, and are shown as the dashed-blue line in Fig. 2.1. The GCR differential flux values are taken from the ISO-15390 model for GCR flux at solar minimum, and are shown are shown as the red line in Fig. 2.2.

$\begin{array}{rcc}\text { Table 4.3: } & \text { SPE and GCR differential flux } \\ & \text { Particle Differential Flux Values }\left(\frac{\#}{s \cdot M e V}\right) \\ \text { Energy } & \text { SPE } & \text { GCR } \\ 1 \mathrm{MeV} & 6.28 \mathrm{e} 8 & 9.40 \mathrm{e}-3 \\ 10 \mathrm{MeV} & 1.25 \mathrm{e} 7 & 1.01 \mathrm{e} 0 \\ 100 \mathrm{MeV} & 1.26 \mathrm{e} 4 & 1.88 \mathrm{e} 1 \\ 1 \mathrm{GeV} & 1.01 \mathrm{e} 1 & 1.51 \mathrm{e} 1 \\ 10 \mathrm{GeV} & 1.00 \mathrm{e} 0 & 2.89 \mathrm{e}-1\end{array}$


Using Eq. 4.15, the radiation energy doses absorbed by the payload may be calculated, and are shown in Table 4.4. Where the unshielded payload receives radiation energy doses of $1.56 \mathrm{e} 3$ and 1.41e1 from SPEs and GCRs, respectively, the shielded payload receives substantially less radiation energy. The radiation dose values are in units of $\frac{J}{s}$, referring specifically to the kinetic energy absorbed per second by the payload when subjected to isotropic radiation environments with flux values given by the SPE and GCR models, respectively. It must be noted that this radiation dose refers to the physical absorbed quantity of radiation energy, which can differ substantially from what a specific biological system or organism would absorb when subjected to the same environment. For the purposes of comparing the effectiveness of differing shielding configurations, the exact quantities are of much less concern than the corresponding ratios between them.

\begin{tabular}{rcc}
\multicolumn{3}{c}{ Table 4.4: Radiation dose absorption } \\
& Radiation energy dose \\
Shielding Configuration & SPE & GCR \\
Control & $1.56 \mathrm{e} 3$ & $1.41 \mathrm{e} 1$ \\
5 Toroids & $3.81 \mathrm{e} 2$ & $1.11 \mathrm{e} 1$
\end{tabular}

Finally, the shielding effectiveness scores may be calculated, and are shown in Table 4.5. The control payload, with no shielding, will by definition have a score of 1 for both SPEs and GCRs, which represents no reduction in radiation dose. The shielded payload receives a score of 4.11 for SPE radiation dose reduction, meaning that it absorbs the same dose of radiation energy in 4.10 time units that the unshielded control payload absorbs in 1 time unit. Similarly, the score of 1.28 for GCR radiation dose reduction means that the shielded payload absorbs the same dose of radiation energy in 1.28 time units that the unshielded control payload absorbs in 1 time unit. 
These scores may be converted to Solar Particle Event and Galactic Cosmic Ray radiation dose reductions of approximately $76 \%$ and $22 \%$, respectively.

\begin{tabular}{rcc}
\multicolumn{3}{c}{ Table 4.5: Shielding effectiveness scores } \\
& Shielding Effectiveness Score \\
Shielding Configuration & SPE & GCR \\
Control & 1 & 1 \\
5 Toroids & 4.11 & 1.28
\end{tabular}

Note that for simplicity and clarity, the number of energy levels tested in this sample simulation run is much smaller than the number used to gather the actual data for this project. Therefore, the doses and scores listed above will be different than those calculated for the same configurations from the actual data.

\subsection{Testing}

To ensure that the data gathered by this virtual experiment is as representative as possible of what data would be gathered from a physical manifestation of the same experiment, $\mathrm{C}++$ unit tests are employed to ensure the functionality of both highlevel and low-level components of the program.

\subsubsection{Geometric Testing}

The unit tests validating ray-tracing, interpolation, and speed/energy conversion functions fall under the category of geometric testing, and seek to ensure the accuracy of the lower-level functions that are used to generate important outputs which are then used by the higher-level functions. The tests listed below have all been passed using a unit testing framework provided by Microsoft Visual Studio 2017. 
The length of the intersection between several particles and a cylinder tested to ensure the proper path length is calculated. Because the radiation dose absorbed by the payload from a particle is highly dependent on that particle's intersection path length, it is important that the path length is accurate for all cases. The test cases verify the intersection path's accuracy for the following cases where the particle:

- enters and exits the cylinder's side

- enters through an endcap and exits through the other endcap while aligned with the cylinder's axis

- enters through an endcap and exits through the other endcap while NOT aligned with the cylinder's axis

- enters and exits through the cylinder's endcaps' edges

- enters through an endcap and exits through the side

The linear 1D-interpolation function, which is used to read stopping power values when a particle enters the payload or the wall, is tested for accuracy. The out-ofbounds behavior is also tested to ensure that out-of-bounds values return 0 .

The conversions between speed and energy are tested. First, the conversion from speed to energy is tested to ensure the following:

- A speed of 0 gives an energy value of 0

- A nonzero speed value will result in different energy values for relativistic and non-relativistic calculations

Next, the conversion from energy to speed is tested to ensure that an energy value of 0 will result in a speed value of 0 . 


\subsubsection{Object Testing}

The higher-level components of the code govern particle equations of motion and energy absorption, and fall under the category of object testing. The purpose of the object unit tests are to verify and validate the two primary functions of the $\mathrm{C}++$ simulation program:

1. Simulate charged particle motion through confined magnetic fields

2. Accurately calculate the kinetic energy absorbed by an object as a charged particle passes though it

Protons with energies between $1 \mathrm{MeV}$ and $10 \mathrm{GeV}$ are simulated being shot at a payload through a $10 \mathrm{~cm}$-thick wall of water. Protons with less than approximately $480 \mathrm{MeV}$ of energy should be fully absorbed by $10 \mathrm{~cm}$ of liquid water, meaning none of their energy would be absorbed by the payload. Similarly, protons with greater than approximately $480 \mathrm{MeV}$ of energy should make it through $10 \mathrm{~cm}$ of liquid water[28], meaning they will still have some kinetic energy left over for the payload to absorb. The following particles and expected results are tested:

- a $1 \mathrm{MeV}$ particle should be fully absorbed by the water, and should NOT deposit energy in the payload.

- a $10 \mathrm{MeV}$ particle should be fully absorbed by the water, and should NOT deposit energy in the payload.

- a $100 \mathrm{MeV}$ particle should be fully absorbed by the water, and should NOT deposit energy in the payload.

- a $1 \mathrm{GeV}$ particle should NOT be fully absorbed by the water, and should deposit energy in the payload.

- a $10 \mathrm{GeV}$ particle should NOT be fully absorbed by the water, and should deposit energy in the payload. 
In a similar fashion, the above test is run, but with with particles impacting the payload and wall on intersection surfaces, and with a smaller, $1 \mathrm{~cm}$-thick wall. At this thickness, particles below approximately $33 \mathrm{MeV}$ will be fully absorbed by the wall and not deposit any energy into the payload.

The last set of tests confirming the accuracy of the energy deposition model ensures that particles are absorbed the the payload as expected. A test payload of radius $0.5 \mathrm{~m}$, height $1 \mathrm{~m}$, composed of amorphous carbon, and with a density of $0.2 \frac{\mathrm{g}}{\mathrm{cm}^{3}}$ is subjected to bombardment by particles across the energy spectrum. At this thickness and payload density, particles with energies less than approximately $160 \mathrm{MeV}$ should be fully absorbed by the payload.

To verify the accuracy of particle motion inside the toroidal solenoids, protons with energies ranging from $1 \mathrm{MeV}$ to $20 \mathrm{GeV}$ are placed in a toroidal solenoid and subjected to the following scenarios and tests:

- Trajectory in a large toroid, normal to the magnetic field: movement is confirmed to be circular with the appropriate gyroradius as calculated using Eq. 4.17 and with the corresponding period of gyration.

- Trajectory in a large toroid, with normal and parallel components to the magnetic field: particle curves in the appropriate direction as determined by Eq. 4.6 .

- Trajectory in a large toroid, velocity initially parallel to magnetic field: initial Lorentz force on particle is confirmed to be 0 , gradually increases in appropriate direction as determined by Eq. 4.6 as the particle propagates forward.

- Trajectory into and out of a normal-sized toroid, oblique: particle travels into toroid at an angle sufficient to be deflected away, particle is confirmed to be 
moving in the approximate appropriate direction, using its appropriate gyroradius and direction of deflection using Eqs. 4.17 and 4.6.

\subsubsection{Statistical Variance Testing}

The simulations run for this thesis used 100 randomly-distributed particles per energy level. To show that this is an adequate number of particles per energy level and does not give rise to significant statistical fluctuations, otherwise identical simulations was run using the nominal 100 and 1,000 particles per energy level, using the 5 toroid shield configuration showcased in the Sample Simulation section. The energy absorption graphs are plotted in Fig. 4.5. While some variation is evident, it is insignificant relative to the overall trends that both simulation runs exhibit. The 1,000-run simulation is very indicative of the true behavior uniform particle placement distribution. Furthermore, the effect on radiation dose reduction is negligible, as seen in Table 4.6.

Note that there is a small peak in absorption at $10 \mathrm{MeV}$. Similar small peaks are seen in other energy absorption graphs presented in this thesis, and while statistical variance may provide some influence, their presence even at 1,000 particle per energy level indicates an underlying physical cause. The physical phenomenon which creates these peaks and valleys in the energy absorption curves is the balancing of two physical facts which counteract each other: a particle's gyroradius increases roughly linearly with the square root of energy, and the stopping power of a material on that particle is non-linearly dependent on its energy. If the performance curves created for this thesis purely measured whether or not a particle was successfully deflected, rather than the energy absorbed by that particle, the performance curves would be smoother and have fewer peaks even at high sample sizes. However, the introduction of a process with inherent non-linearities such as radiation transport gives rise to these irregularities. 


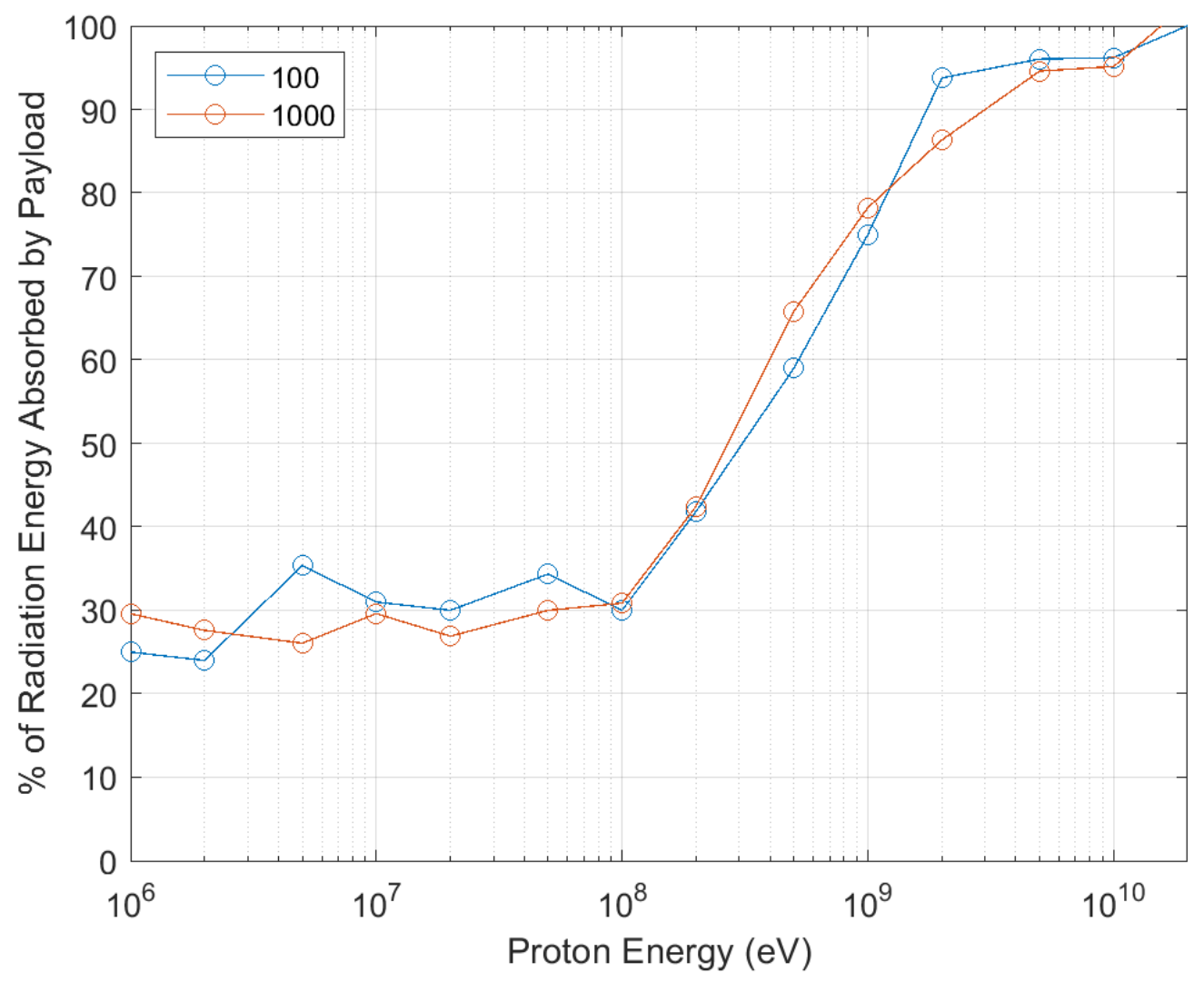

Figure 4.5: Statistical variance test

Table 4.6: Statistical variance test

Shielding effectiveness score

$\begin{array}{rcc}\text { \# of Particles } & \text { SPE } & \text { GCR } \\ 100 & 3.02 & 1.10 \\ 1,000 & 3.22 & 1.12\end{array}$


Chapter 5

RESULTS

\subsection{Overview}

To generate the results analyzed, 21 total simulations were run to evaluate the effectiveness of material shielding and electrodynamic toroidal shielding at reducing the radiation experienced by a an Apollo CSM-sized cylindrical spacecraft, referred to as "the payload," from SPEs and GCRs. The 21 simulations are listed as follows:

- 1 Control - no shielding

- 5 Water shielding

- 15 Toroidal shielding

- 5 Geometric configurations

- 3 Current levels per geometric configuration

The payload itself is a cylinder with a radius of 2.5 meters, height of 10 meters. For the purposes of particle absorption, it is modeled as being made of amorphous carbon with density of $0.2 \frac{\mathrm{g}}{\mathrm{cm}^{3}}$, which is $10 \%$ of amorphous carbon's given density of $2 \frac{g}{\mathrm{~cm}^{3}}[28]$. The purpose of this materials configuration, and specifically the low material density, is to simulate a spacecraft where humans inhabit about $10 \%$ of its volume and are equally likely to be at any particular interior point.

For the below comparisons of payload radiation energy absorption across the energetic particle spectrum, proportions are given as a percentage of the radiation energy absorbed by an unshielded payload, which is shown in Fig. 5.1. As seen, all of each 
particle's kinetic energy is entirely absorbed by the payload for particle energies below $2 \mathrm{GeV}$. Above $2 \mathrm{GeV}$, particles have enough energy so that, in some cases, they can travel through the payload without depositing all their kinetic energy in the payload. Note that the lower limit of the figure's y-axis is at $80 \%$.

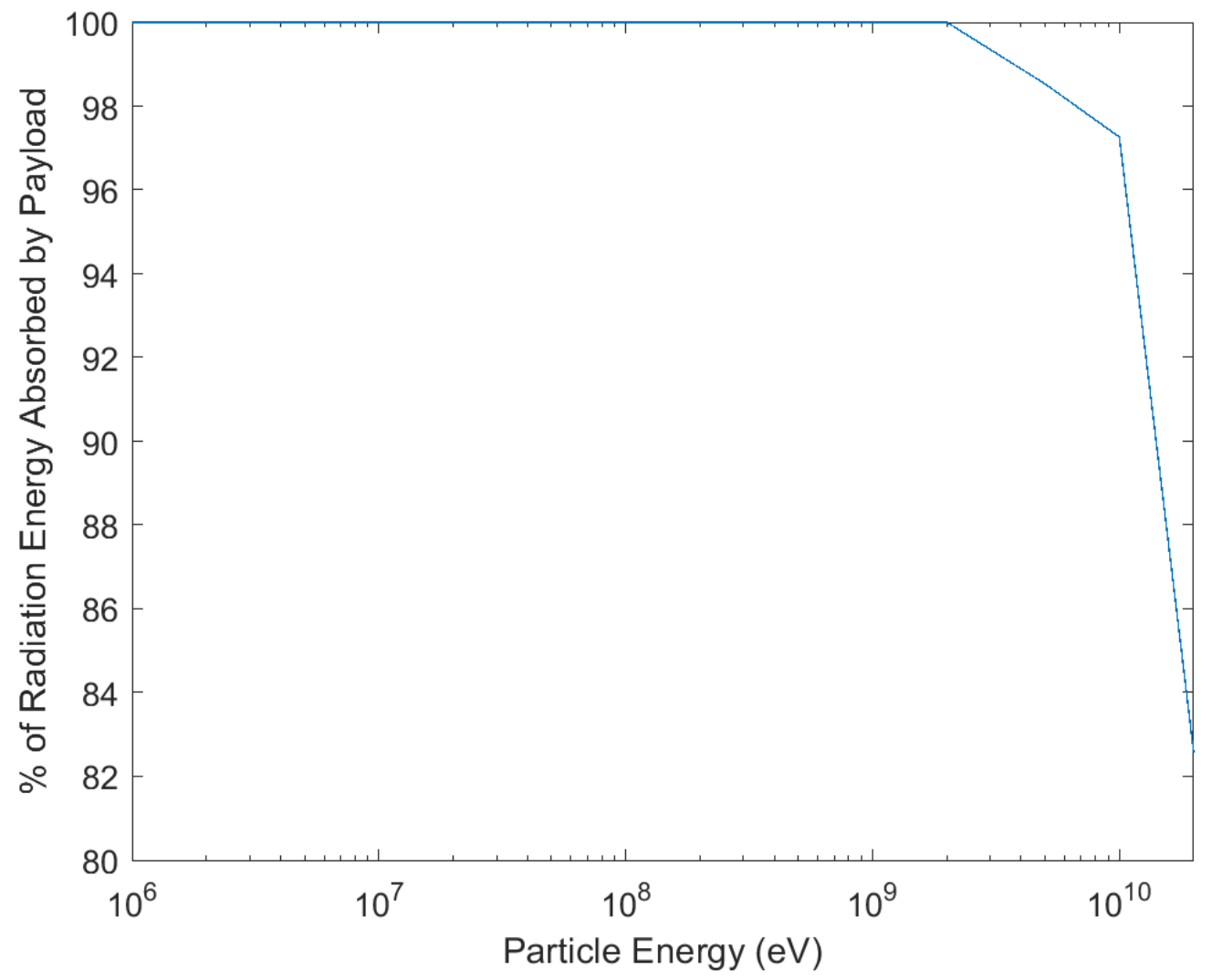

Figure 5.1: Unshielded control payload

\subsection{Particle Deflection Performance}

\subsubsection{Water Shielding}

The simulations depicted in Fig. 5.2 show how much radiation energy can penetrate a uniformly-thick shield of water to be absorbed by a payload behind it, normalized 
against the control simulation. Water shielding will slow down all particles that pass through it, and completely absorb particles below a certain energy level, dependent on the shield's minimum thickness.

For particles with kinetic energies greater than $1 \mathrm{MeV}$ and less than $200 \mathrm{MeV}$, a $1 \mathrm{~cm}$ shield of water will absorb all of the kinetic energy, but absorbs almost none of the kinetic energy from particles with energies greater than $200 \mathrm{MeV}$. By comparison, a $10 \mathrm{~cm}$ shield of water completely protects the payload from particles with energies up to $100 \mathrm{MeV}$, but provides minimal protection from particles above $500 \mathrm{Mev}$. Shields of thickness between those two extremes follow a similar pattern; water shields offer complete protection from particles below a certain energy level, dependent on the shield thickness, but are ineffective against higher energy particles. 


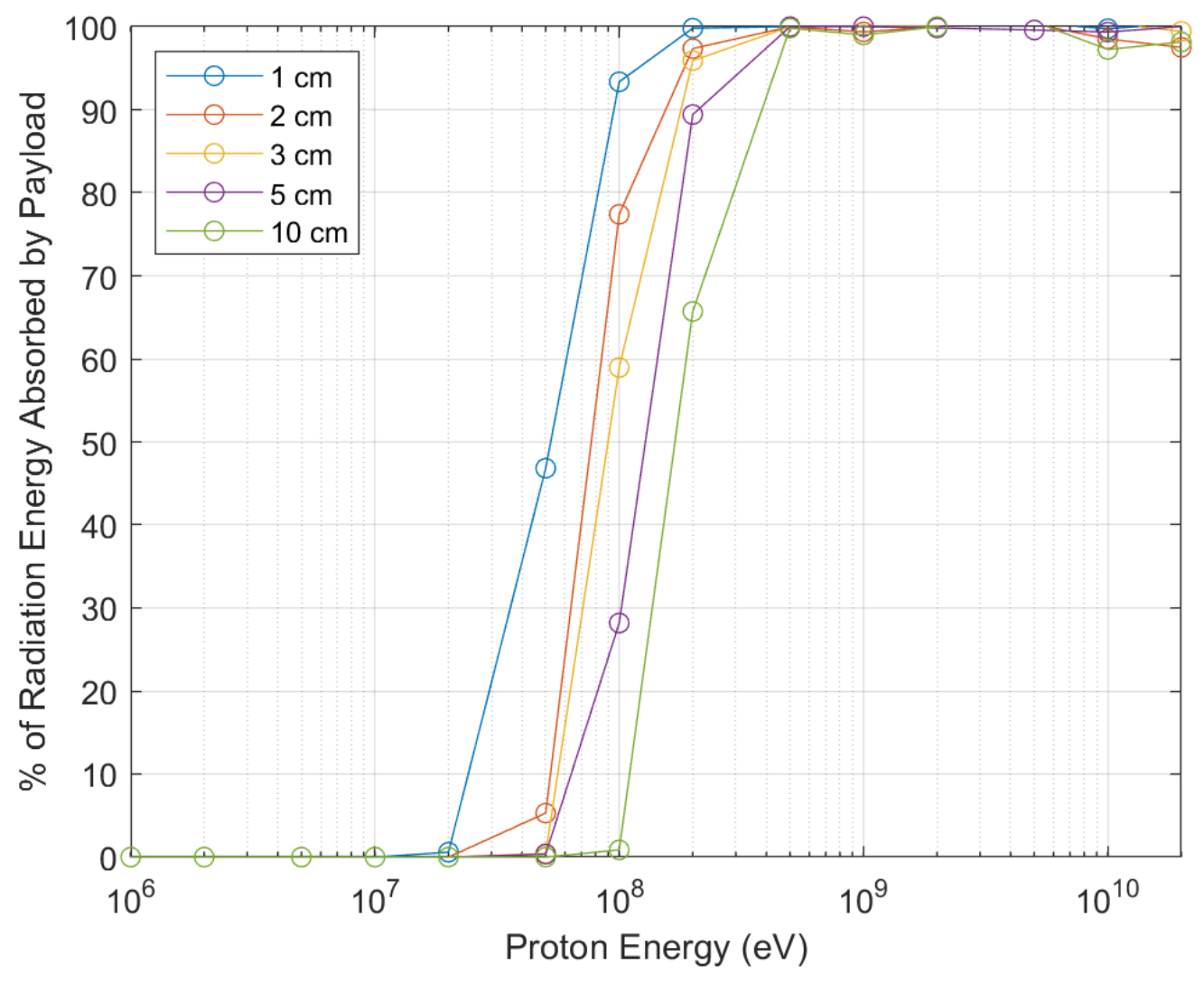

Figure 5.2: Water shielding

\subsubsection{Toroidal Shielding}

Fig. 5.3 similarly depicts the effectiveness of the electrodynamic toroidal shielding at the nominal current level and magnetic field strength.

The configuration labels used in the legends and tables of the Results section refer to specific geometric shielding configurations and are described in Table 5.1. All toroidal solenoids have 1,000 turns and are given the same nominal current level of $30 \mathrm{kA}$. 


\title{
Label Description
}
A 5 primary toroids
B 5 primary toroids + endcap toroids
C 5 primary toroids + endcap toroids + nested toroids
D 10 primary toroids
E 10 primary toroids + endcap toroids
C* $\quad \mathrm{C}+$ a 1 cm-thick water shield
$\mathrm{E}^{*} \quad \mathrm{E}+\mathrm{a} 1 \mathrm{~cm}$-thick water shield

\section{Table 5.1: Description of shielding geometric configurations}

\begin{abstract}
All the simulated configurations are at least fairly effective at deflecting particles of energies below about $100 \mathrm{MeV}$, and are minimally effective against particles with energies of $2 \mathrm{GeV}$ or above. The most obviously effective configuration is $\mathrm{C}$, which is the most effective configuration at deflecting particles with energies between $20 \mathrm{GeV}$ and $2 \mathrm{GeV}$. This band of the energy spectrum makes up the bulk of GCR protons in the model used for this thesis, therefore it is unsurprising that configuration $\mathrm{C}$ performs better than the other configurations against GCRs, as will be shown in the Radiation Dose Reduction Effectiveness section.
\end{abstract}




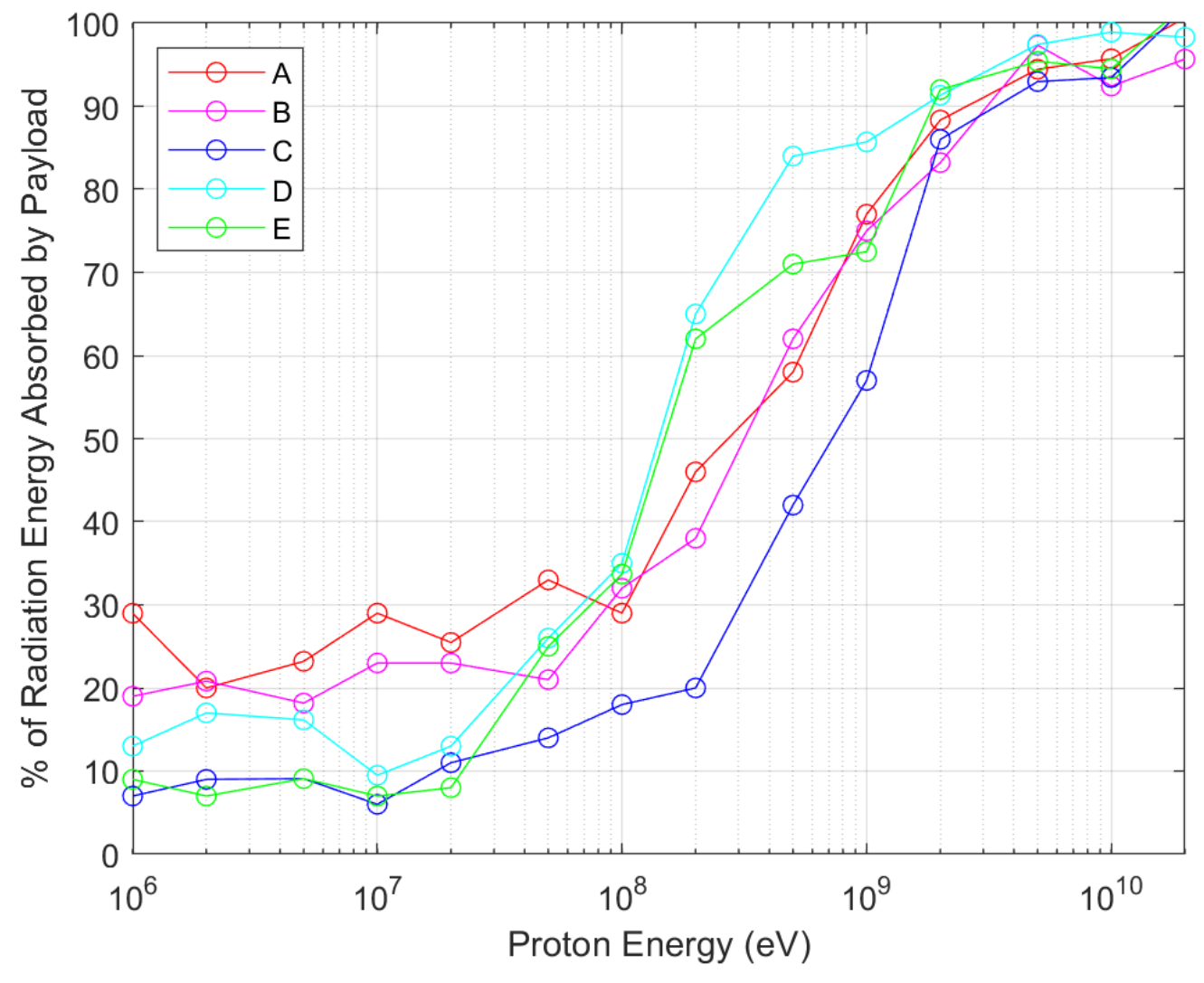

Figure 5.3: Toroidal shielding, $\mathrm{B}=1.7 \mathrm{~T}$

The toroids simulated to create the data depicted in Fig. 5.3 sustain a current of $30 \mathrm{kA}$ in solenoids with 1,000 turns, resulting in magnetic field strengths of approximately 1.7 $\mathrm{T}$, varying slightly with toroid primary radius. These currents and magnetic fields are on the order of those achievable by present-day high-temperature-superconductor technology[30]. However, as more research is done in the fields of superconductivity, and specifically high-temperature-superconductivity, magnetic fields attainable may rise substantially. This could be accomplished by increasing either the current in the solenoids, the number of turns in each solenoid, or a combination of both. To see how increases in these values would affect the performance of the shielding configurations depicted above in Fig. 5.3, the same simulations were run, where each geometric 
configuration was run at three current levels, creating three different magnetic field strengths:

- $30 \mathrm{kA}$, the nominal current level (I): magnetic field (B) strength of $1.7 \mathrm{~T}$

- $150 \mathrm{kA}, 5 \times I: \mathrm{B}=8.5 \mathrm{~T}$

- $300 \mathrm{kA}, 10 \times I: \mathrm{B}=17 \mathrm{~T}$

The results of these simulations are shown in Fig. 5.4 and 5.5, each showing the performance of toroidal shields with magnetic field strengths of $8.5 \mathrm{~T}$ and $17 \mathrm{~T}$, respectively. For identical geometric configurations, higher currents are shown to provide better protection against particles of a given energy level. As with the performance curves at $1.7 \mathrm{~T}$, configuration $\mathrm{C}$ still performs the best, followed by $\mathrm{E}$. 


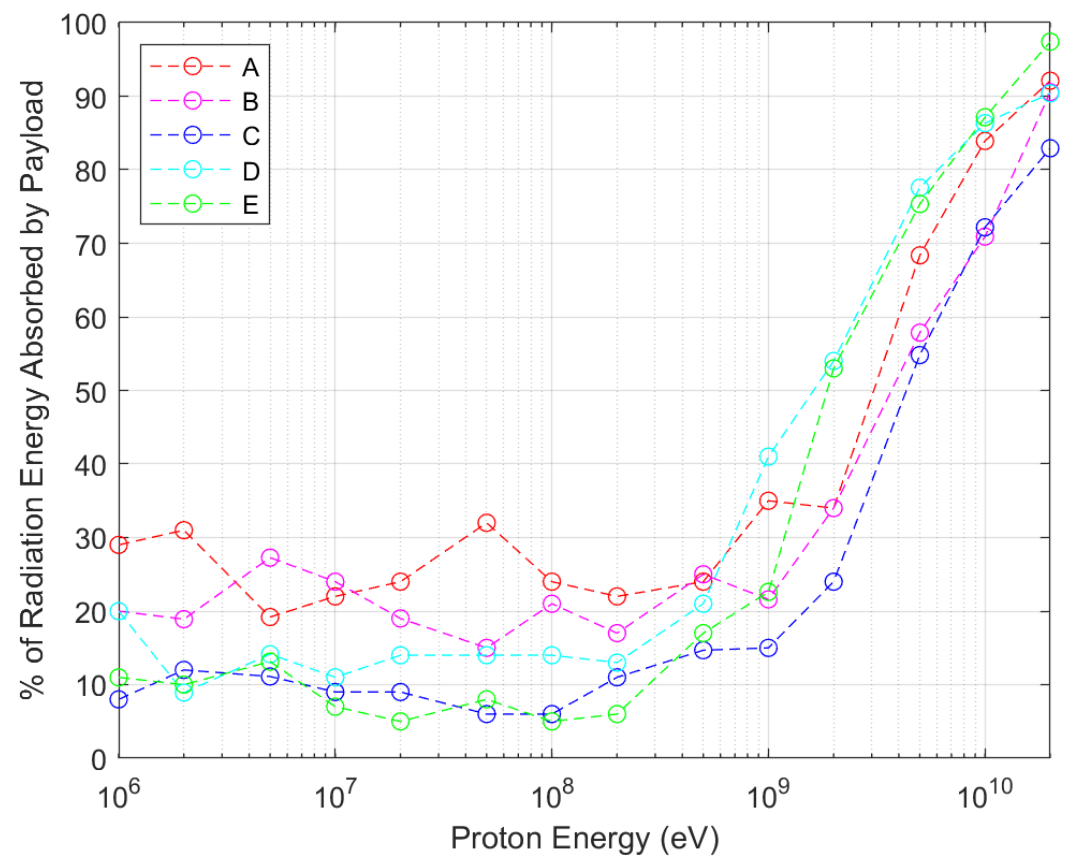

Figure 5.4: Toroidal shielding, $\mathrm{B}=8.5 \mathrm{~T}$

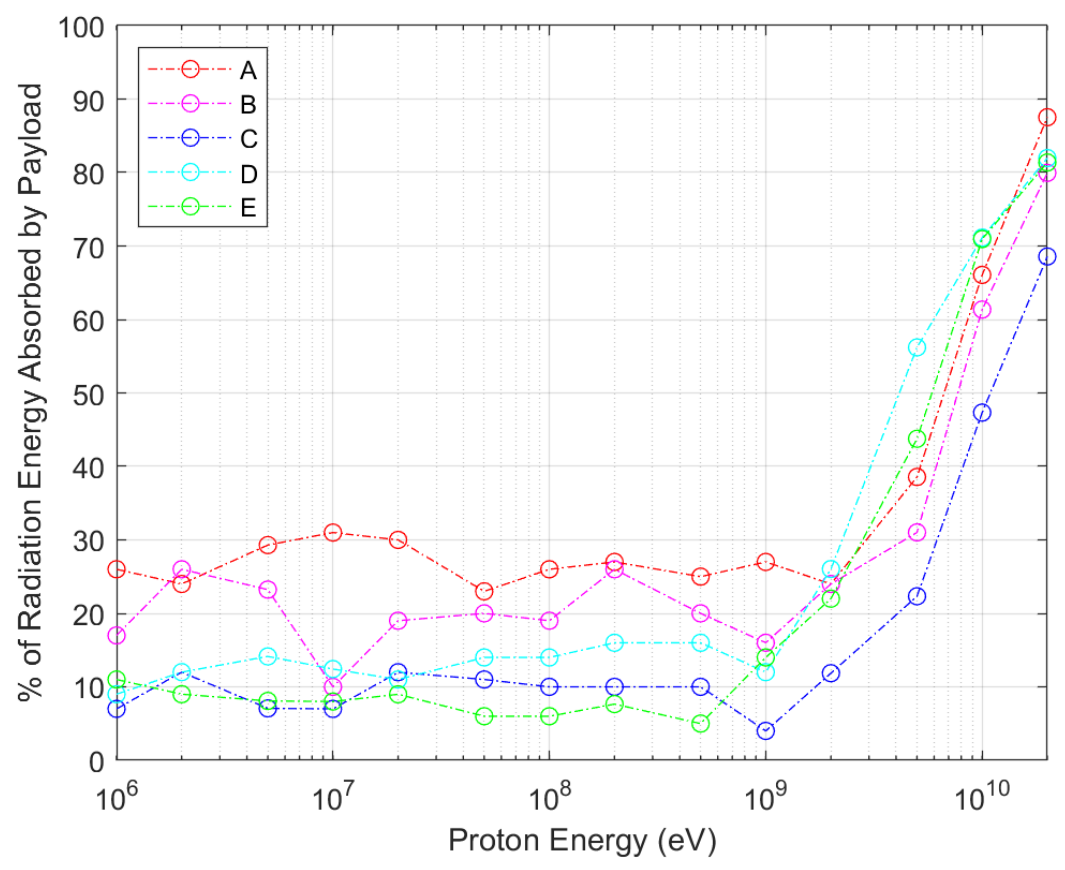

Figure 5.5: Toroidal shielding, $\mathrm{B}=17 \mathrm{~T}$ 
Configuration $\mathrm{C}$ performs the best across the particle energy spectrum at the nominal current level. A rendering of this configuration is shown in Fig. 5.6. When the currents of all the geometric configurations are increased it still performs the best, and its performance curves are shown in Fig. 5.7. Where this configuration at the nominal current level still let through $60 \%$ of incoming radiation energy from particles with energies of $1 \mathrm{GeV}$ (compared to an unshielded payload), the same configuration at 10 times the nominal current level cuts this number to less than $10 \%$.

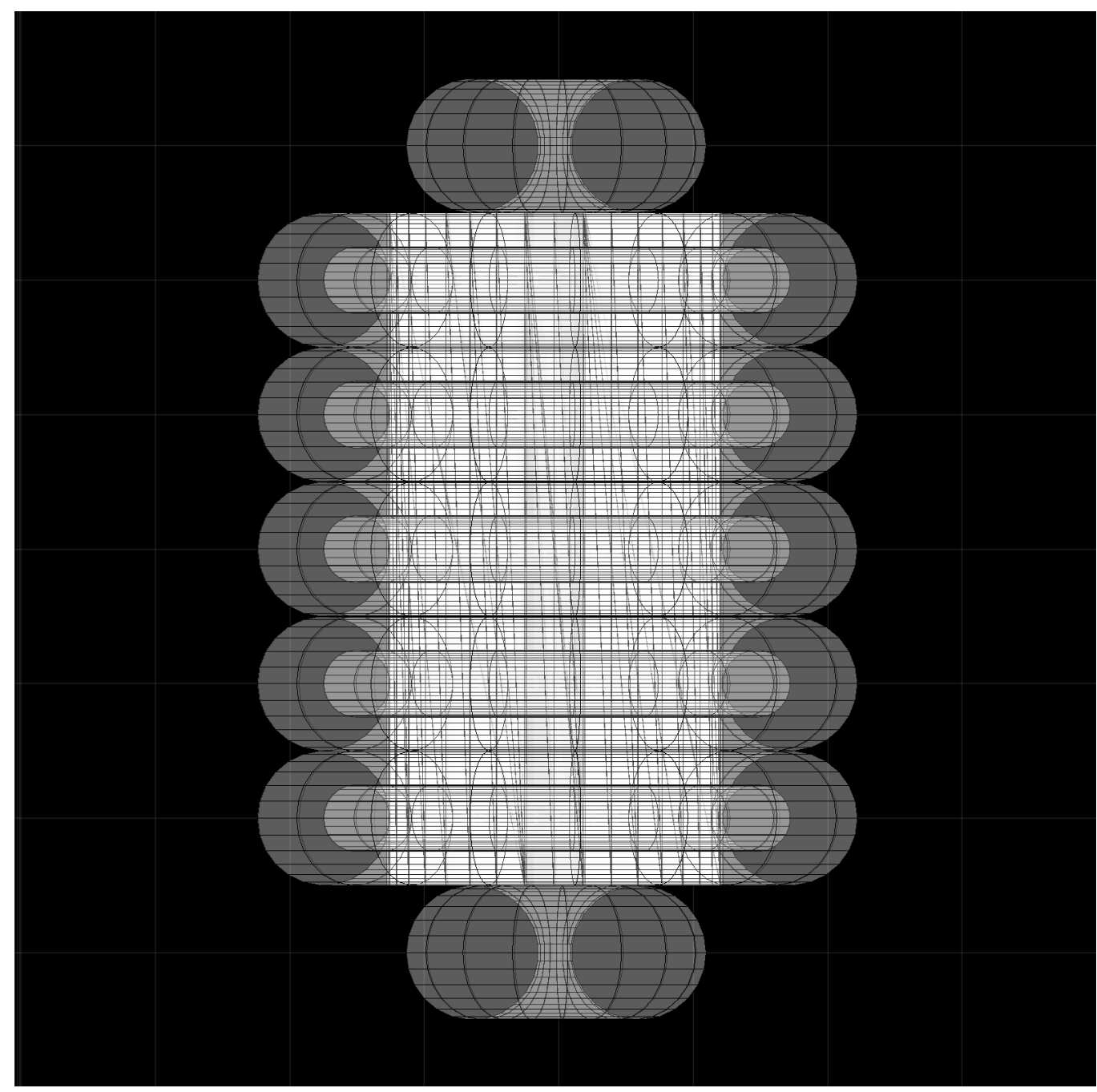

Figure 5.6: Configuration C Rendering 


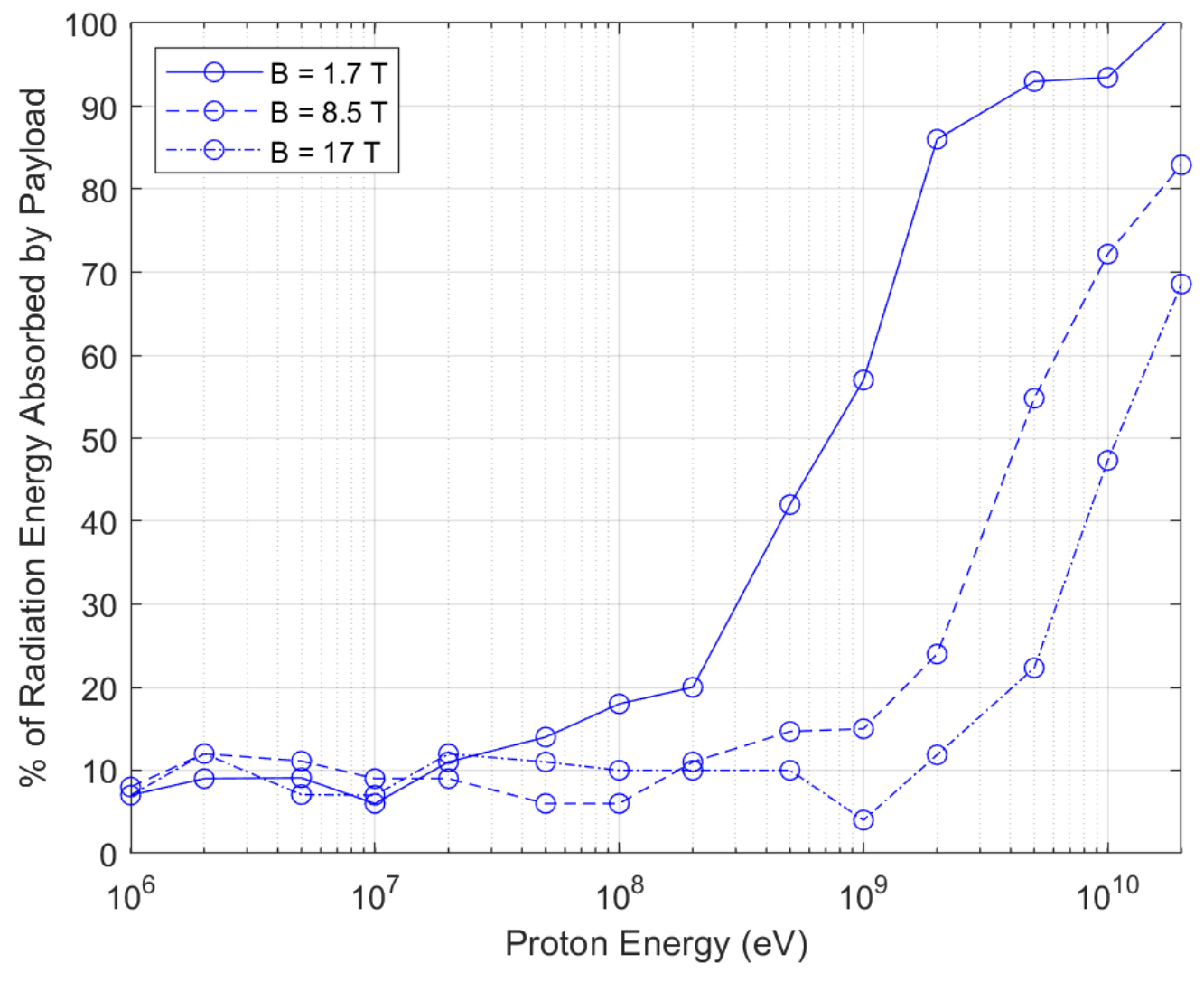

Figure 5.7: Configuration C

The second-best shielding configuration is that of Configuration E, owing its success to very good performance against lower-energy particles, only letting through half as much radiation energy as Configurations A or B. Configuration E is rendered in Fig. 5.8 and its performance curves are shown in Fig. 5.9. At a magnetic field strength of $17 \mathrm{~T}$, configuration E manages to keep the payload radiation energy absorption to below $10 \%$ for particles below $500 \mathrm{MeV}$, which includes the entire SPE spectrum of the model used in this thesis. E performs fairly well against higher energy particles at a field strength of $17 \mathrm{~T}$, although not quite as well as configuration $\mathrm{C}$. 


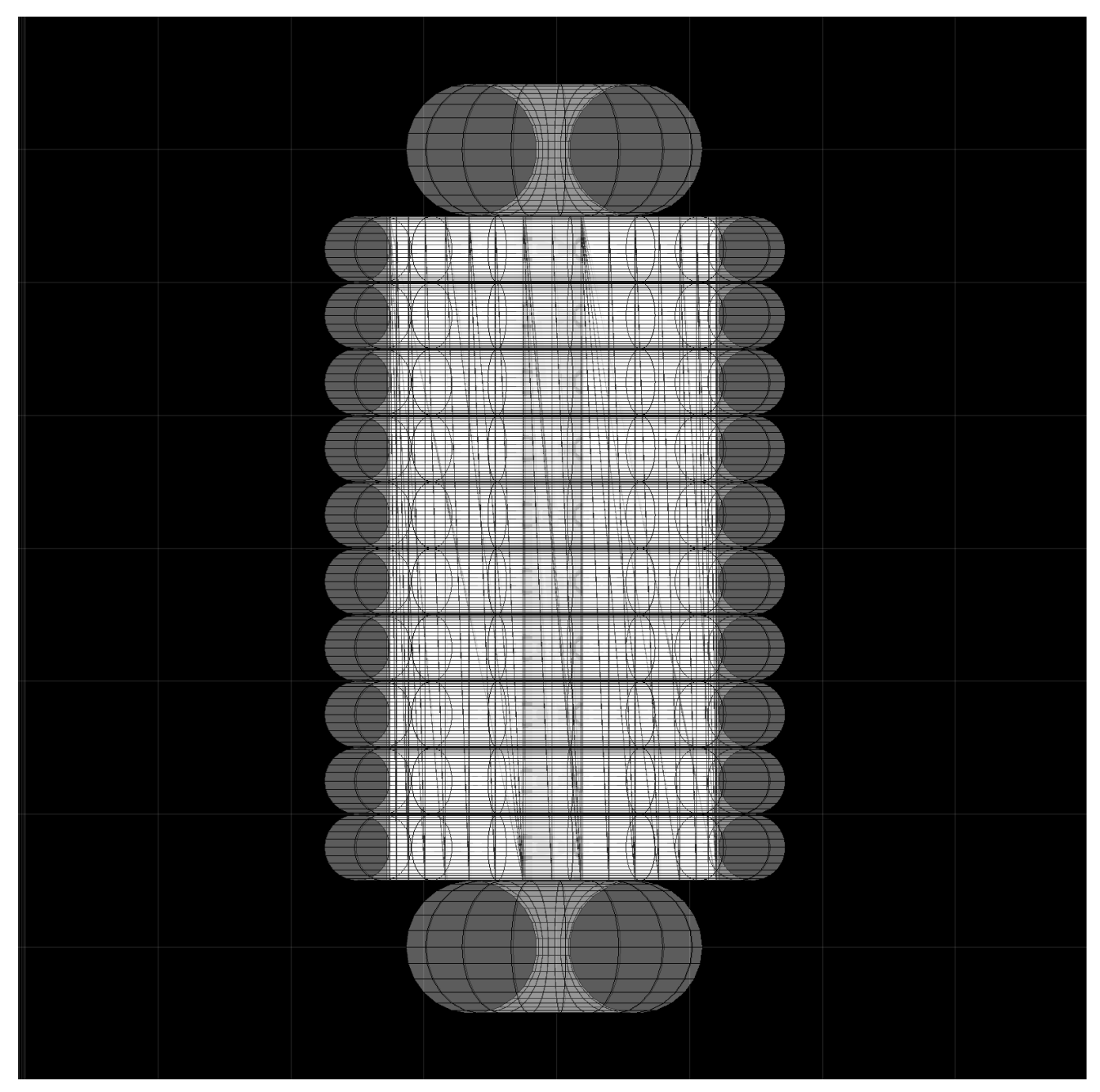

Figure 5.8: Configuration E Rendering 


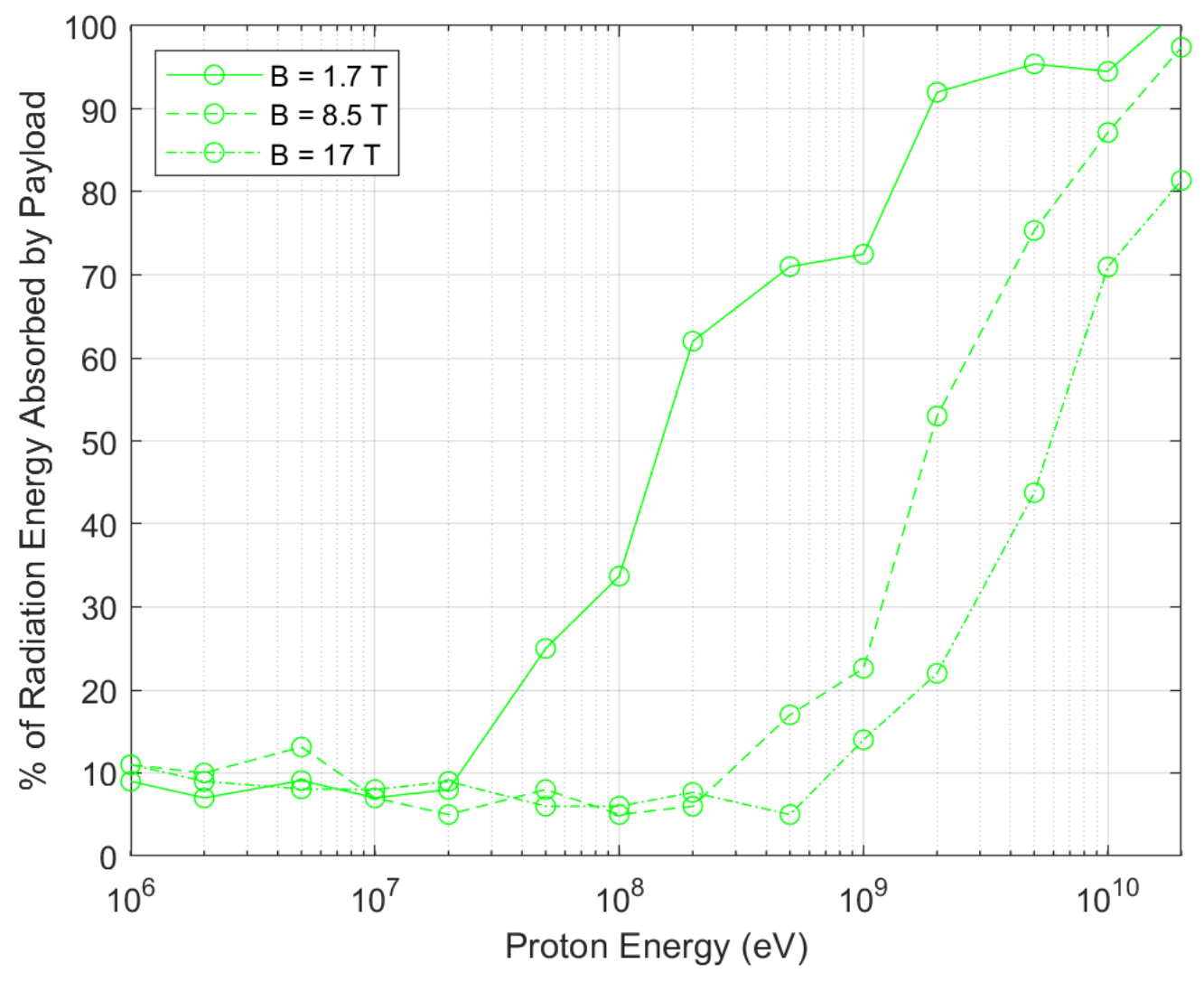

Figure 5.9: Configuration E

\subsubsection{Combined Water and Toroidal Shielding}

As Fig. 5.2 and Figs. 5.3-5.5 show, water shielding appears to better shield against lower energy particles (below $20 \mathrm{MeV}$ at $1 \mathrm{~cm}$ of water) while toroidal shielding can sustain significant protection against particles up to $100 \mathrm{MeV}$, or even $5 \mathrm{GeV}$ when higher current levels are used. To combine these advantages of both types of shielding, the two best-performing shielding configurations, $\mathrm{C}$ and $\mathrm{E}$, were added to a payload with a $1 \mathrm{~cm}$-thick water shield. These configurations are labeled $\mathrm{C}^{*}$ and $\mathrm{E}^{*}$, respectively, and the performance curves for both these configurations at all 3 magnetic field strengths are shown in Fig. 5.10 and Fig. 5.11, respectively. 
As seen in Figs. 5.10 and 5.11, the combined configurations protect entirely against particles below $20 \mathrm{MeV}$, which matches the performance of $1 \mathrm{~cm}$ of water shielding, and retain the effectiveness against higher-energy particles that is seen in the toroidal shielding configurations, particularly at higher current levels. This means that combining a small amount of material shielding with electrodynamic provides complete protection against energetic protons up to a certain energy level, determined by the capabilities of the material shielding, and retains nearly identical performance against higher-energy particles.

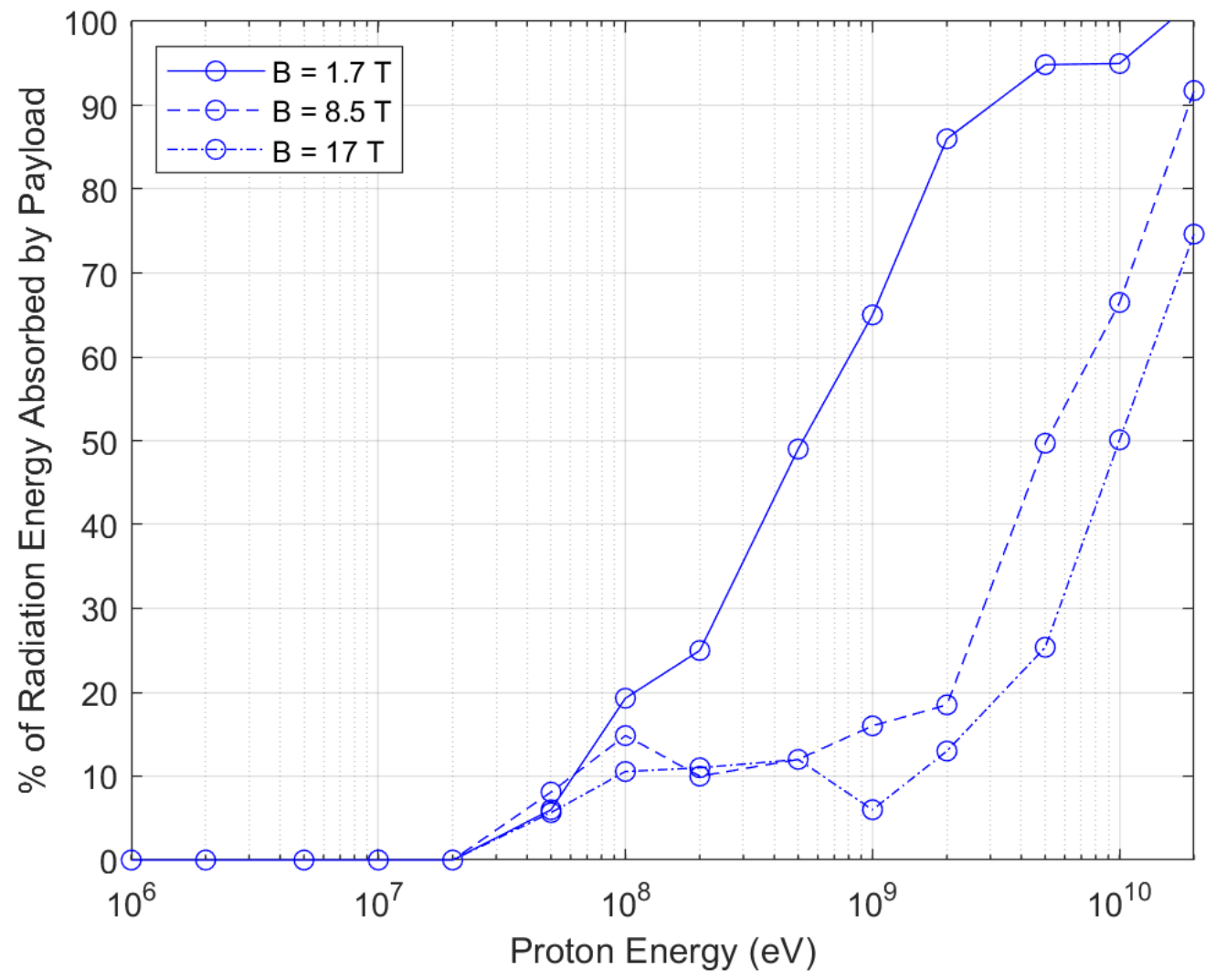

Figure 5.10: Configuration $\mathrm{C}^{*}$ 


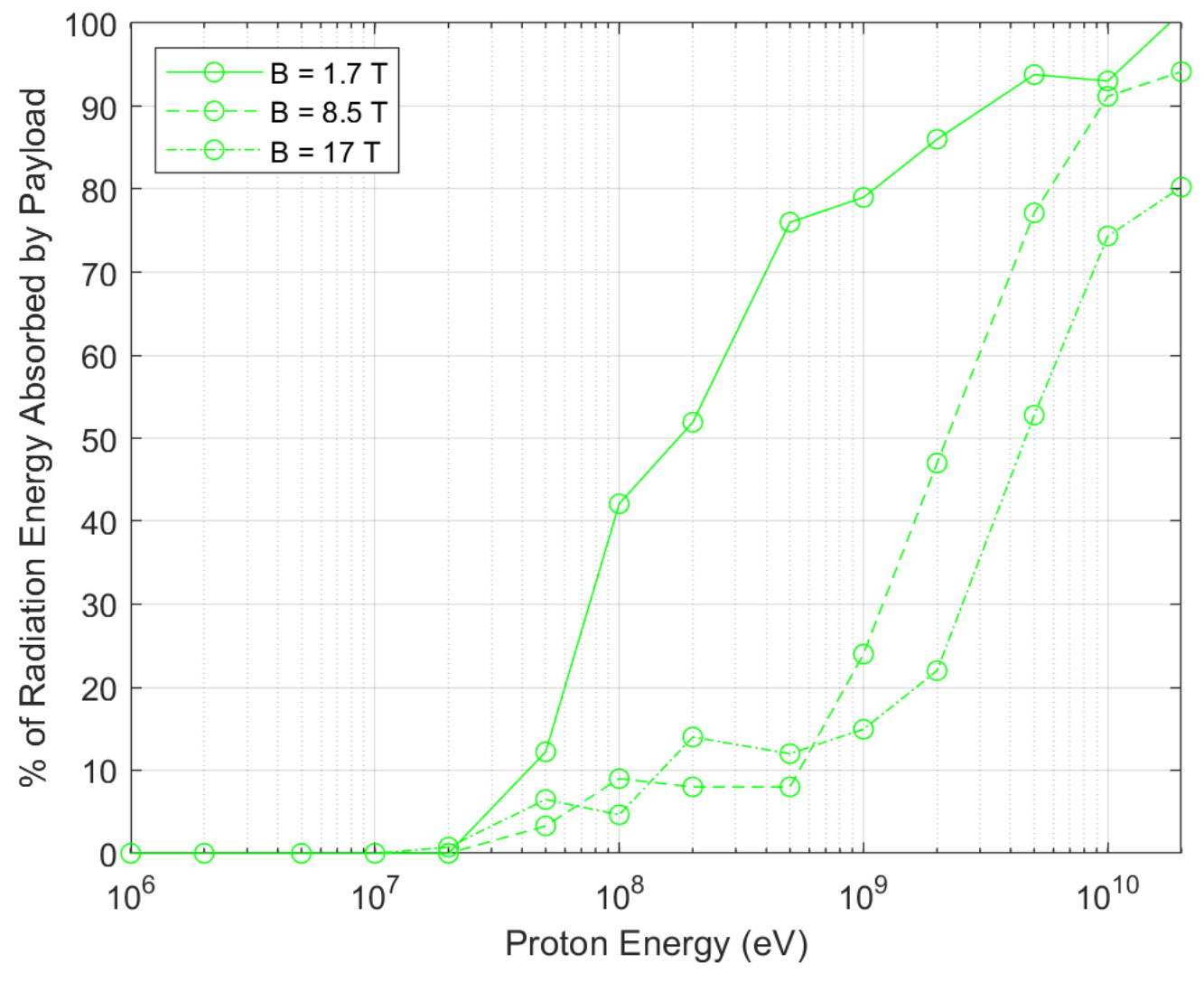

Figure 5.11: Configuration $\mathrm{E}^{*}$

\subsection{Radiation Dose Reduction Effectiveness}

While the above figures describe how effective each shielding configuration is at reducing the radiation energy absorbed from particles of particular energies, the below figures factor in the relative abundance of energetic particles across the energy spectrum to estimate each shielding configuration's dose reduction in various radiation environments.

The differential flux curves depicted in Fig. 2.1 and Fig. 2.2 were used to separately evaluate each shielding configuration's radiation dose reduction in the Solar Particle Event radiation environment and in the Galactic Cosmic Ray radiation environment. 
The shielding effectiveness scores shown in Fig. 5.12 through Fig. 5.15 represent the reciprocal of the radiation dose rate received in a given radiation environment while shielded as a proportion of the dose rate received while not shielded. A score of 1 indicates the dose rate is identical to that received while not shielded. A score of 2 represents a $50 \%$ reduction in the dose rate while shielded compared to the dose rate while not shielded, and thus while shielded it takes twice as long to receive the same total dose of radiation than it would without shielding.

\subsubsection{Water Shielding}

Figs. 5.12 and 5.13 depict the shielding effectiveness of various thicknesses of water surrounding the payload for both the Solar Particle Event and Galactic Cosmic Ray radiation environments. For a Solar Particle Event subjecting a hypothetical spacecraft to radiation particles matching the 7-year maximum probable fluxes in Fig. 2.1, the shielding effectiveness score shows a general trend of exponential growth with increasing shield thickness. As seen in Table $5.2,1 \mathrm{~cm}$ of water reduces the radiation dose from such an event by a factor of 7.1, and a $10 \mathrm{~cm}$ thick shield of water reduces the experienced radiation by a factor of over 17.1. For the same spacecraft experiencing the typical Galactic Cosmic Ray radiation environment as depicted in 2.2, water shielding provides minimal practical shielding. While GCR shielding effectiveness does demonstrate a slight trend of linear increase, even a $10 \mathrm{~cm}$ thick water shield would reduce the radiation dose from the GCR environment by only $1 \%$. 


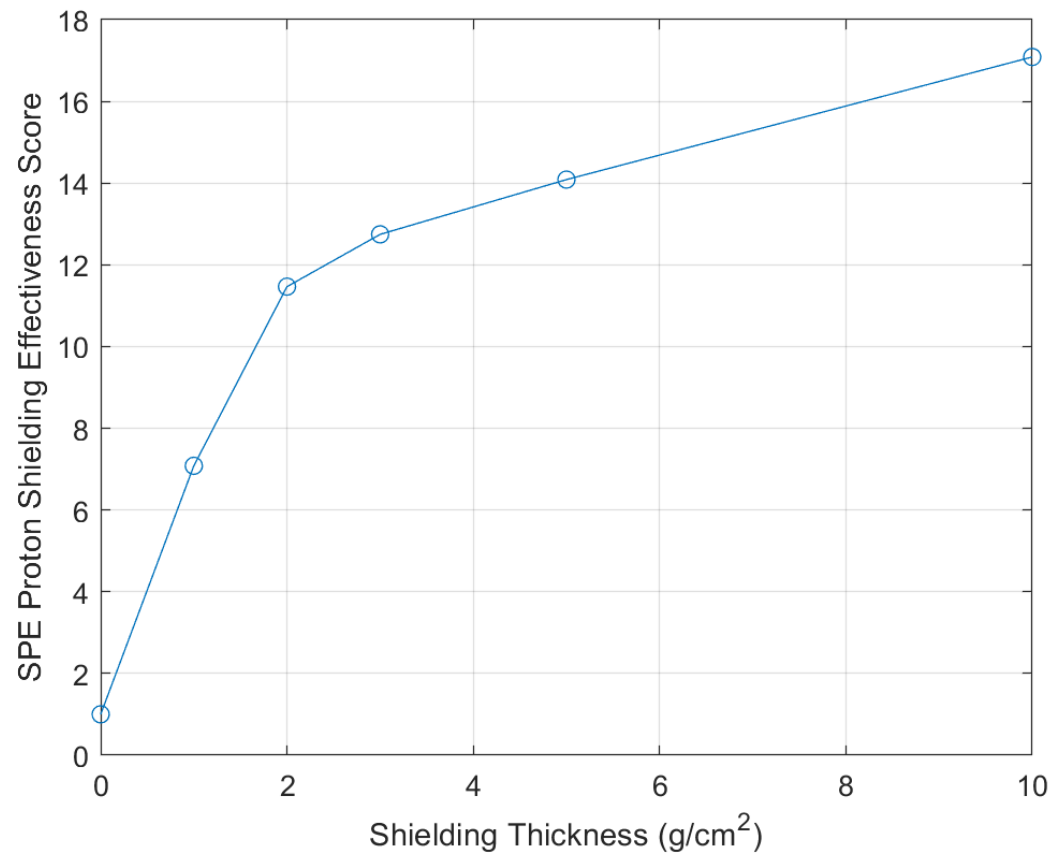

Figure 5.12: Water shielding vs. SPEs

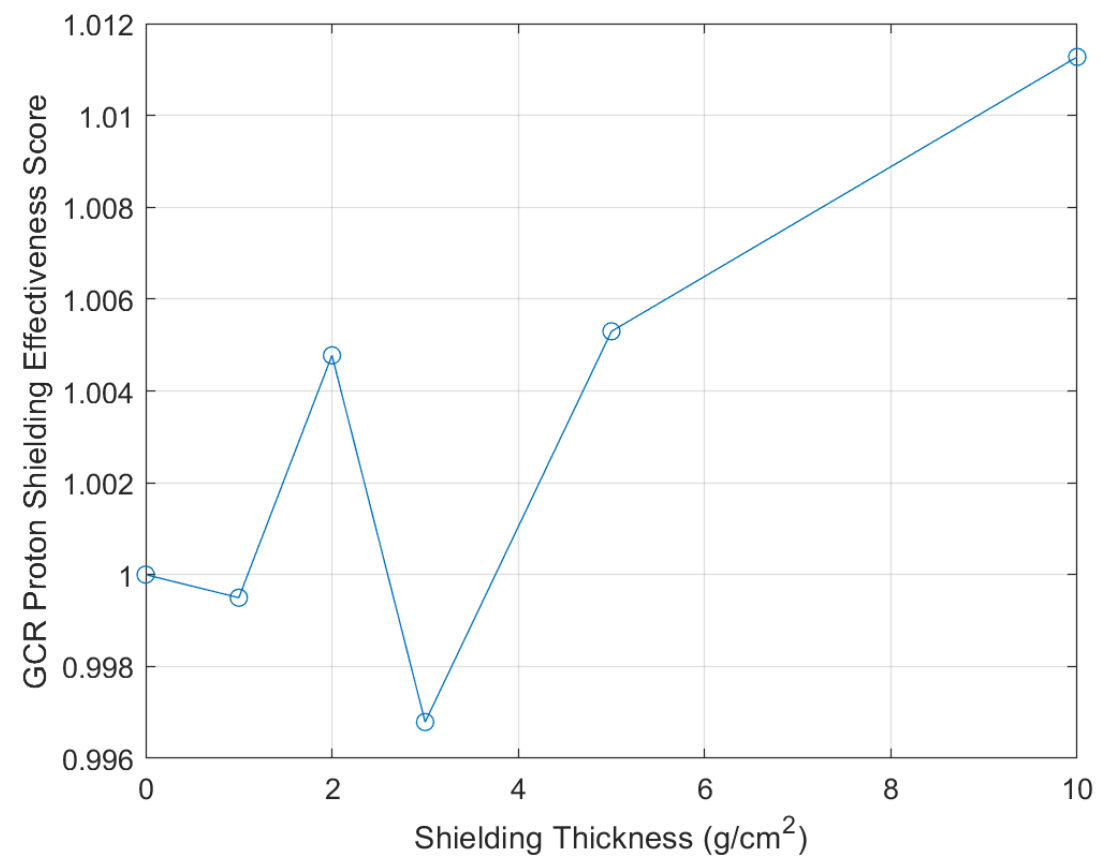

Figure 5.13: Water shielding vs. GCRs 


\section{Table 5.2: Water shielding}

\section{Water Shielding Effectiveness Score}

$\begin{array}{rcc}\text { Shielding Thickness }(\mathbf{c m}) & \text { Solar Particle Event } & \text { Galactic Cosmic Rays } \\ 0 \text { (Control) } & 1.0 & 1.000 \\ 1 & 7.1 & 0.999 \\ 2 & 11.4 & 1.005 \\ 3 & 12.7 & 0.997 \\ 5 & 14.1 & 1.005 \\ 10 & 17.1 & 1.011\end{array}$

\subsubsection{Toroidal Shielding and Combined Shielding}

The shielding effectiveness scores of the electrodynamic toroidal shielding configurations and current levels are similarly depicted below in Table 5.3. The shielding effectiveness scores for toroidal shielding in the SPE radiation environment are depicted in Fig. 5.14, and for the GCR radiation environment in Fig. 5.15. In these two figures, each line corresponds a particular geometric configuration, and each point on a given line corresponds to that same geometric configuration run with a different current level. The x-axis is in units of magnetic volume $\left(\mathrm{Tm}^{3}\right)$, which is calculated for each configuration by summing the product of each toroid's magnetic field strength with its volume, as shown in Eq. 5.1. The addition of $1 \mathrm{~cm}$ of water to configurations $\mathrm{C}$ and $\mathrm{E}$ greatly increases effectiveness against SPEs, but actually marginally decreases effectiveness against GCRs. Note that $\mathrm{E}^{*}$ achieves similarly high performance against SPEs as $\mathrm{C}^{*}$, but at a much lower magnetic volume (meaning such a system would likely require less material to construct and could operate with less cryocooling). $\mathrm{E}^{*}$ has a lower magnetic volume than $\mathrm{C}^{*}$ because $\mathrm{E}^{*}$ consists of 10 relatively primary small toroids ( + the endcaps), where $\mathrm{C}^{*}$ 's 5 nested toroids are the 
same volume as $\mathrm{E}^{*}$ 's primary toroids, but also has 5 much larger primary toroids that add a significant amount of volume.

$$
V_{m}=\sum_{i}^{N}\left(2 \pi^{2} r_{i}^{2} R_{i}\right) B_{i}
$$

In the Solar Particle Event radiation environment, toroidal shielding does provide substantial protection even at the nominal current level. At this current level, the geometric configuration of 5 primary toroids, 5 nested toroids, and endcap toroids gives a shielding effectiveness score of approximately 7.7, while the other geometric configurations at the nominal current level give shielding effectiveness between 3 and 5. For the first three shielding configurations listed in Table 5.3 and Fig. 5.14 those configurations with 5 large primary toroids - the change in score as current increases to 5 and 10 times the nominal current level is small enough to simply be the result of statistical noise. For the next two configurations - those configurations with 10 smaller primary toroids - the score shows a trend of moderate increase as the current level also increases. The reason that the score does not appear to significantly increase with magnetic volume is because while any of the lower-energy SPE protons that travel through will almost certainly be deflected, there are areas where particles of any energy level can travel through the shielding system and impact the payload relatively unimpeded. These areas are where the individual toroids border each other, and particles travelling through these areas would need to travel through at most several $\mathrm{cm}$ of the toroidal shield's interior magnetic field, enabling these particles to make it through relatively unhindered. 


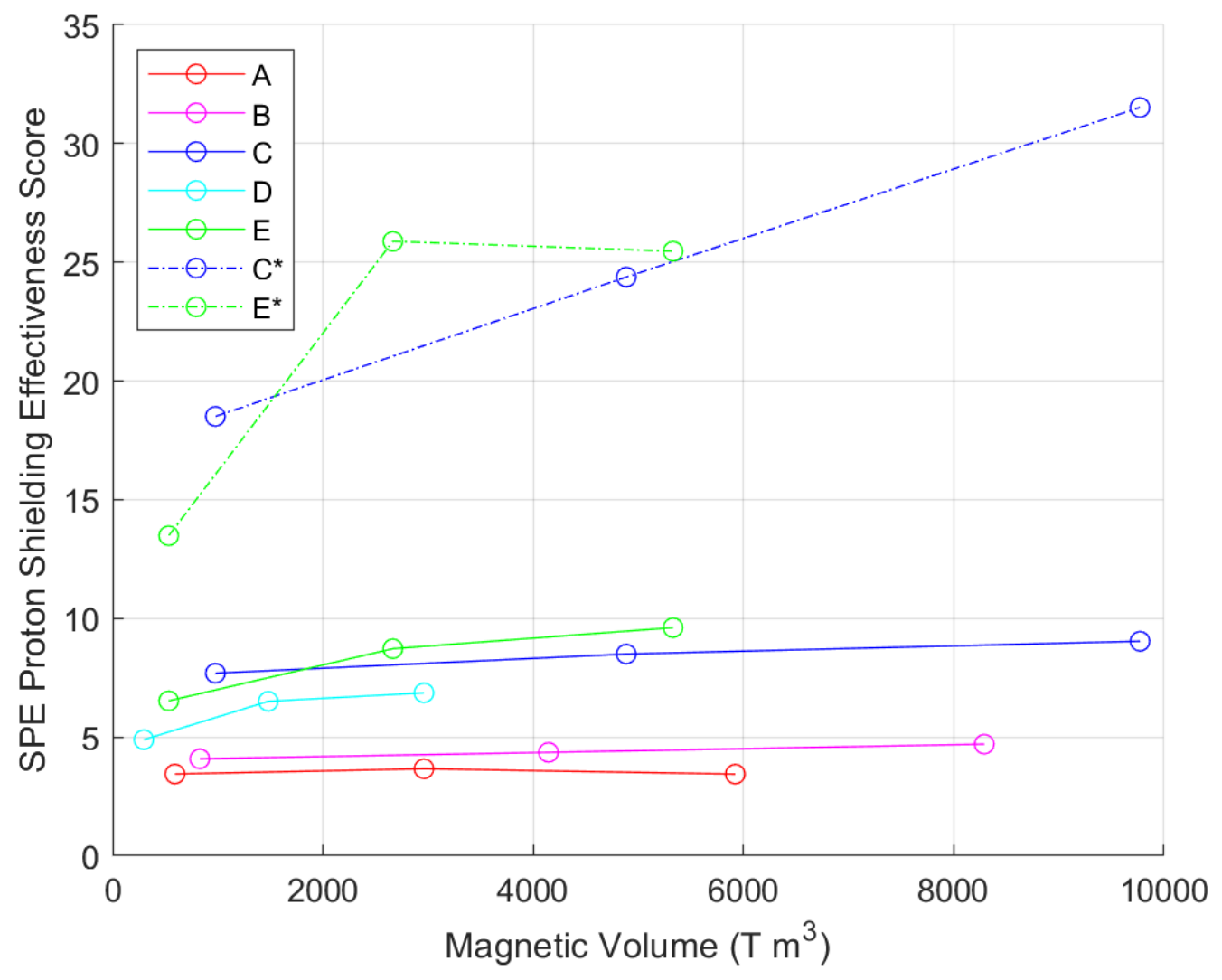

Figure 5.14: Toroidal shielding vs. SPEs

In the Galactic Cosmic Ray radiation environment, toroidal shielding provides minimal protection at the nominal current level (which generates magnetic fields with strengths between 1.5 and 2 Tesla) for any geometric configuration of shielding. However, toroidal shielding emerges as a possible solution to GCR radiation for higher current levels (resulting in magnetic field strengths of up to 20 Tesla). GCR shielding effectiveness scores appear to be increase linearly with magnetic volume; this is to be expected because the gyroradius of a charged particle in a magnetic field is inversely proportional to magnetic field strength[29]. The most effective configuration of those simulated is that of 5 primary toroids, 5 nested toroids, and endcap toroids, which at 10 times the nominal current level gives a GCR shielding effectiveness score of 
4.20. The other geometric configurations at 10 times the nominal current level give substantial GCR shielding effectiveness scores between 2.2 and 2.9.

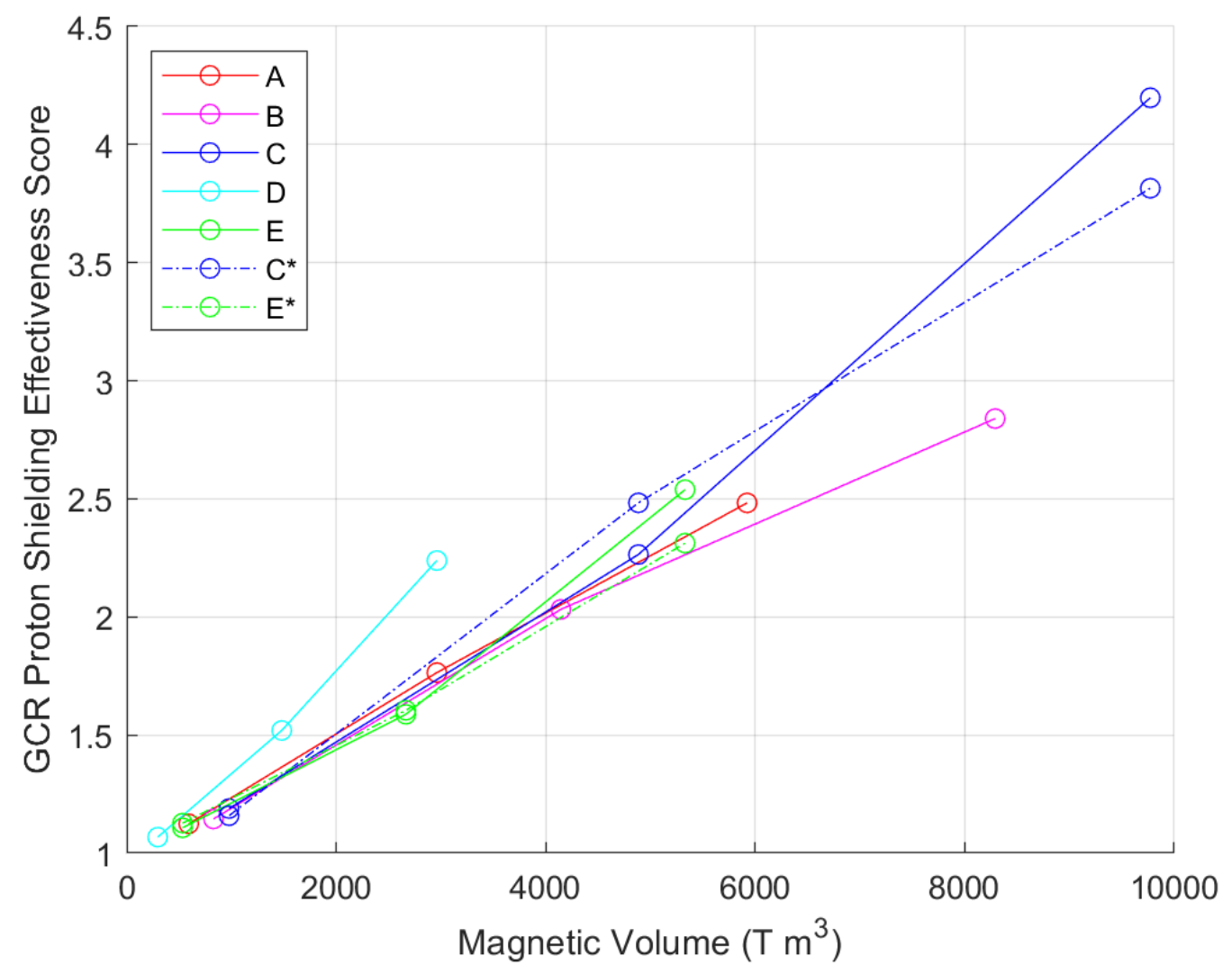

Figure 5.15: Toroidal shielding vs. GCRs

Where the SPE shielding effectiveness was shown to be highly dependent on the geometric configuration of the toroidal shields, the primary driver in GCR shielding effectiveness at the sizes and magnetic field strengths investigated here appears to be the total magnetic volume that the shielding system can provide. This is evidenced by the apparent trend of nearly all the geometric configurations' GCR shielding effectiveness scores, and their corresponding scores at different current levels, falling on the same line when plotted against magnetic volume, with minimal variance. This is evidence that further effectiveness against GCRs could be similarly achieved through 
taking the same geometric configurations and creating even higher strength internal magnetic fields.

\section{Table 5.3: Toroidal shielding} SPE GCR

$\begin{array}{rccccccc}\text { Toroidal Shield Configuration } & I & 5 \times I & 10 \times I & I & 5 \times I & 10 \times I \\ \text { N/A (Control) } & 1.00 & 1.00 & 1.00 & 1.00 & 1.00 & 1.00 \\ \text { A } & 3.44 & 3.66 & 3.43 & 1.12 & 1.76 & 2.48 \\ \text { B } & 4.09 & 4.35 & 4.70 & 1.14 & 2.03 & 2.84 \\ \text { C } & 7.69 & 8.50 & 9.03 & 1.19 & 2.26 & 4.20 \\ \text { D } & 4.89 & 6.50 & 6.86 & 1.07 & 1.52 & 2.24 \\ \text { E } & 6.52 & 8.72 & 9.61 & 1.11 & 1.59 & 2.54 \\ \text { C* } & 18.5 & 24.4 & 31.5 & 1.16 & 2.48 & 3.82 \\ \text { E* } & 13.5 & 25.8 & 25.5 & 1.13 & 1.6 & 2.31\end{array}$

\subsection{Comparison to Past Research}

Gehrke's 2018 master's thesis on water shielding provided a basis on which to compare the findings of this thesis on the effectiveness of water as a means to protect both SPE and GCR radiation[6]. His calculated dose reductions are plotted against water shield thickness for the 1989 SPE in Fig. 5.16 and compared against the corresponding findings from this thesis in Fig. 5.17. Gehrke's thesis found the reduction of SPE radiation dose to follow a much more gradual curve than was found in this thesis. The driver of this difference is likely the different SPE models used. The October 1989 SPE is considered the strongest SPE ever measured[6], and would therefore likely have had particles of higher energies than the SPE model used in this thesis. This addition

of higher energy particles would drastically decrease the steepness of the proportional 
dose rate curve, causing it to look more like that of the proportional dose rate curve for the GCR environment. Gehrke's thesis found water to be effective at shielding against GCRs[6] and is shown in Fig. 5.18, while this thesis found water shielding to be minimally effective against GCRs, which is shown in Fig. 5.19. As with the differences in findings for SPE dose rates, the primary cause is likely the difference in models used; this thesis used a GCR model for solar minimum, which results in the largest and most energetic GCR flux values, making lower thicknesses of water much less effective. The secondary cause of these differences is that Gehrke's thesis used NASA's OLTARIS program to make sophisticated energy deposition calculations, including biological doses, where this thesis merely calculated the physical radiation energy deposition in a simple homogeneous material. 


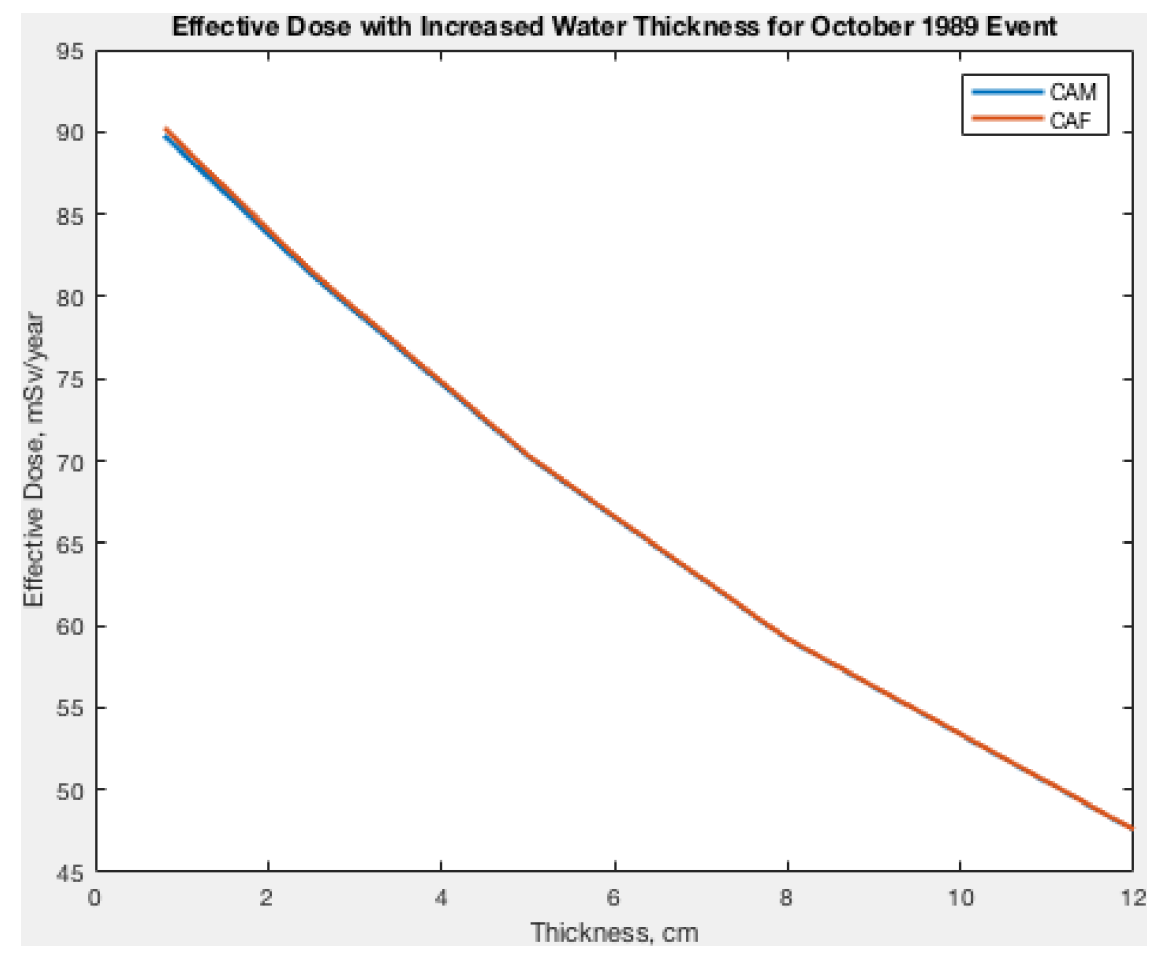

Figure 5.16: Water shielding vs. SPEs, via Gehrke[6]

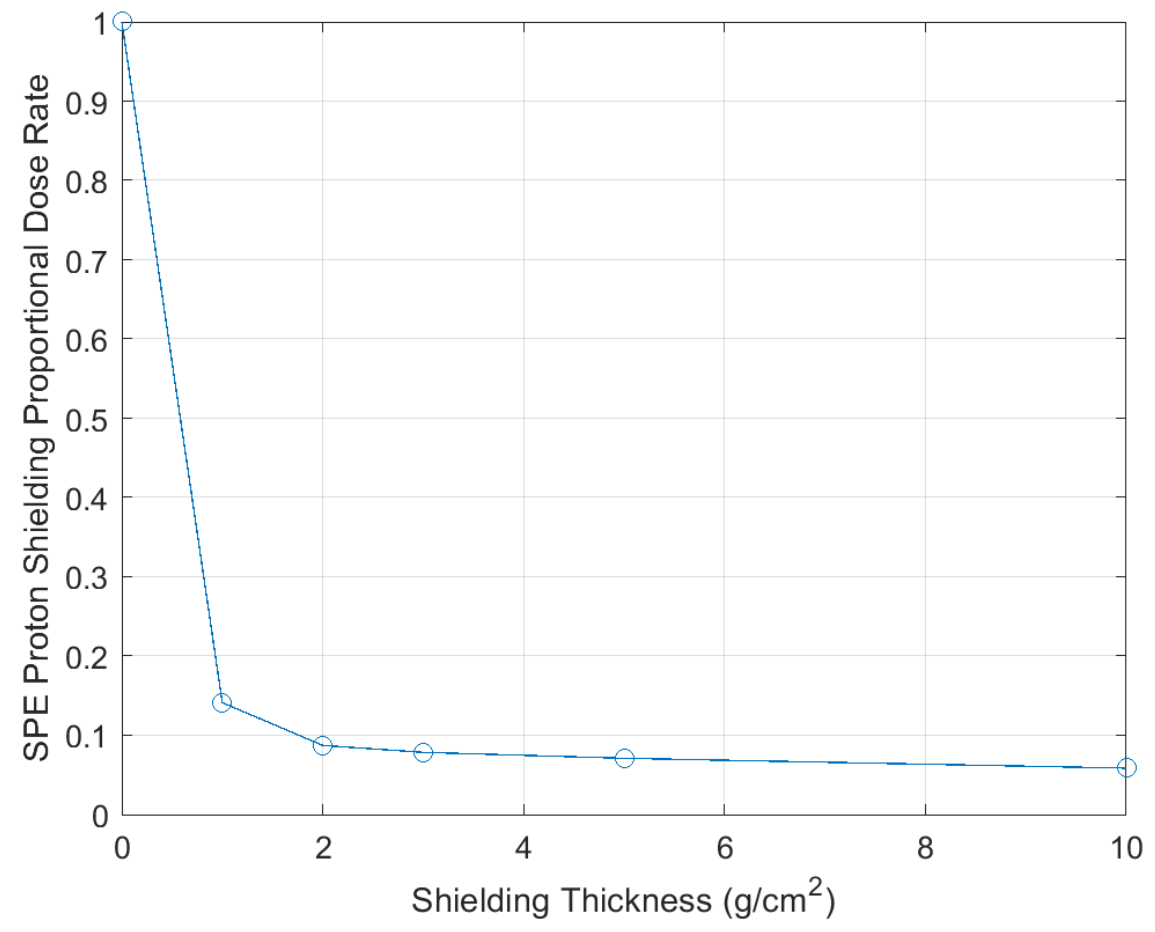

Figure 5.17: Water shielding vs. SPEs 


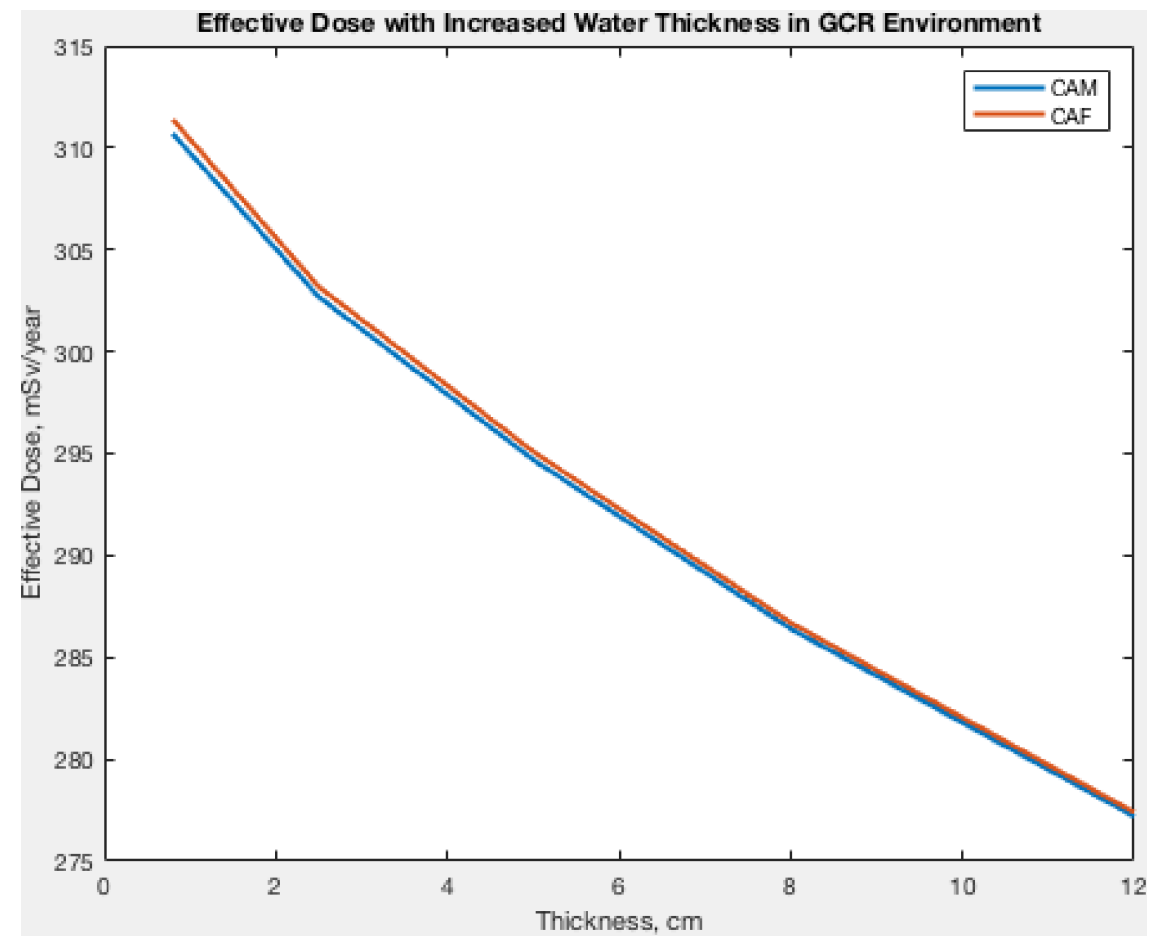

Figure 5.18: Water shielding vs. GCRs, via Gehrke[6]

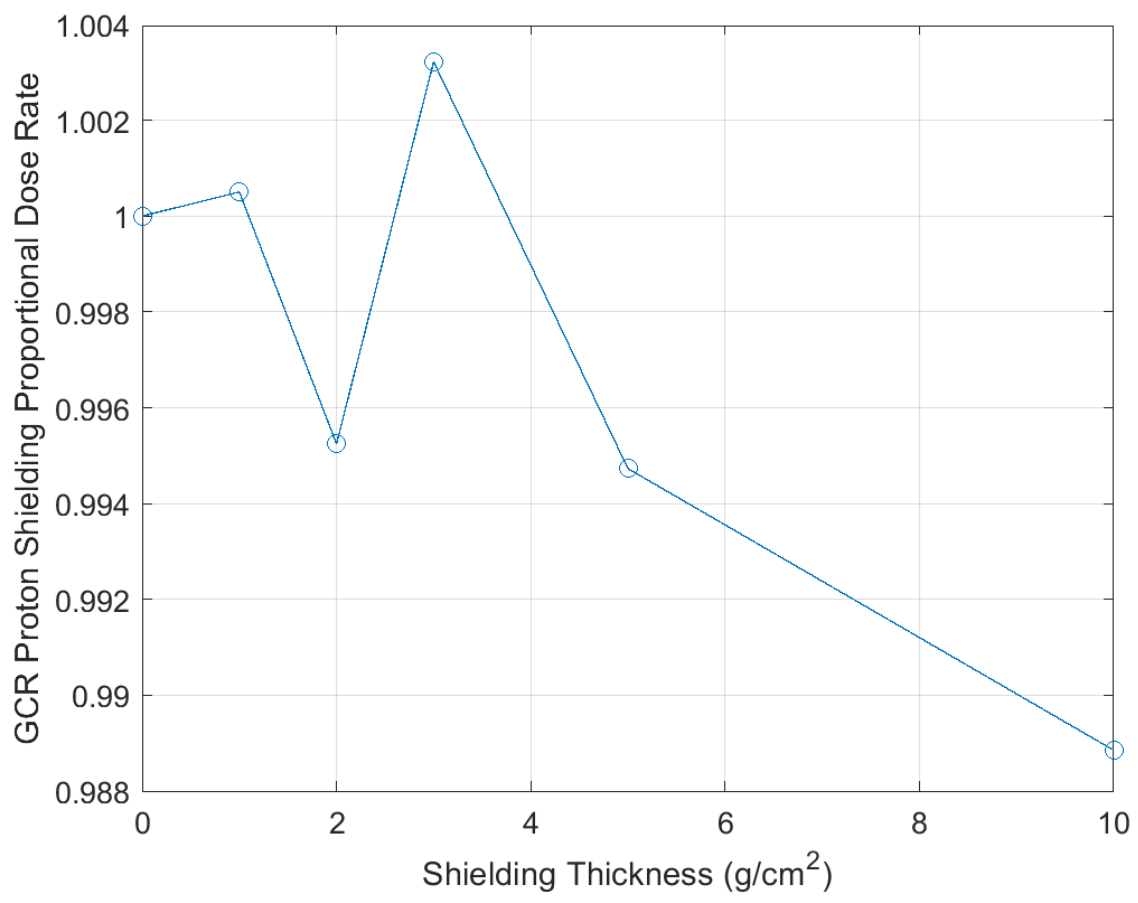

Figure 5.19: Water shielding vs. GCRs 
The best comparison with previous research for the electrodynamic toroidal shielding is Fig. 3.5 from the 2009 article in the Journal of Spacecraft and Rockets[8], which shows shielding effectiveness for magnetic field strengths of $2 \mathrm{~T}, 4 \mathrm{~T}$, and $10 \mathrm{~T}$. While these field strengths are different from the three used in this thesis, they are of the same orders of magnitude and provide a suitable basis for comparison. The shielding effectiveness graph for configuration $\mathrm{D}$ uses primary toroids with inner radii of 0.5 $\mathrm{m}$ and does not use endcap toroids, which best matches the configurations used to create Fig. 3.5, so it is used as the comparative basis. The shielding effectiveness of configuration D, which is shown in Fig. 5.20, performs very similarly to those examined in the article in question at around $200 \mathrm{MeV}$, but relatively less effective at higher energy levels. Overall, the shielding effectiveness shown in Fig. 3.5 falls within $20 \%$ of the shielding effectiveness shown in Fig. 5.20, when differences in magnetic field strength are accounted for. These differences are likely primarily due to this thesis's counting of proportional energy absorbed, rather than the journal article in question's simple counting of what proportion of particles were successfully deflected. Other differences in the results may be accounted for by differences in the target cylindrical spacecraft's dimensions between this thesis and the article in question. 


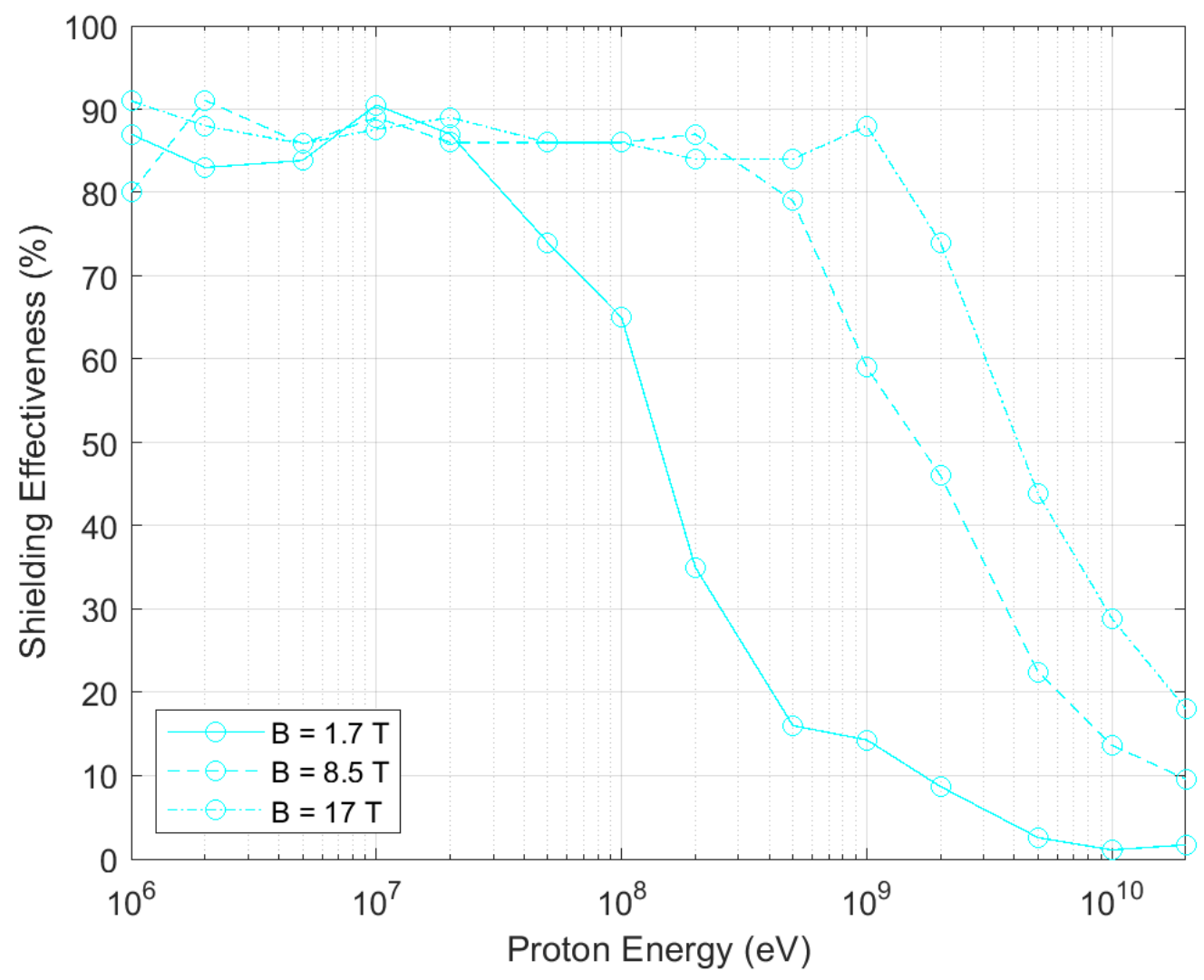

Figure 5.20: Configuration D

\subsection{Results Summary}

The scores and masses of the two highest-scoring toroidal shield configurations and the two combined shielding configurations are shown in Table 5.4 with the scores of the 1-cm-thick and 10-cm-thick water shields for reference. Using the particle simulation and analysis programs, bulk material shielding was shown to be very effective against the lower-energy SPE radiation environment, but not very effective against the higher-energy GCR radiation environment without increasing shielding thicknesses to unrealistic levels. Material shielding's advantage is in its ability to fully stop lower energy particles with only a couple $\frac{g}{\mathrm{~cm}^{3}}$ or less of material, and in 
the ease of creating a wall or shell with which all or part of a habitable spacecraft may be surrounded with no gaps through which radiation particles can travel unimpeded. Comparatively, the electrodynamic shielding configurations were found to be capable of deflecting higher-energy particles, including those found in the GCR radiation environment, especially at high magnetic field strengths. One of the inherent disadvantages of using this type of toroidal electrodynamic shielding is that many toroidal solenoids are required to eliminate areas where radiation particles can travel through without interacting with the solenoids. Possible solutions include using multiple layers of solenoids, but this would double the mass of superconductive material required and significantly increase the space required for the system. A more compact and highly effective was instead shown to be the combination of a modest amount of material shielding, which is primarily effective against SPE particles, with the top two configurations of toroidal shielding, which are primarily effective against GCRs.

Table 5.4: Shielding effectiveness scores

$$
\text { Score Score, } 10 \times I
$$

$\begin{array}{rcccc}\text { Shielding Configuration } & \text { SPE } & \text { GCR } & \text { SPE } & \text { GCR } \\ 1 \text { cm water } & 7.08 & 1.00 & \text { N/A } & \text { N/A } \\ 10 \text { cm water } & 17.1 & 1.01 & \text { N/A } & \text { N/A } \\ \text { C } & 7.69 & 1.19 & 9.03 & 4.20 \\ \text { E } & 6.52 & 1.11 & 9.61 & 2.54 \\ \text { C }^{*} & 18.5 & 1.15 & 31.5 & 3.81 \\ \mathrm{E}^{*} & 13.4 & 1.13 & 25.4 & 2.31\end{array}$


Chapter 6

CONCLUSION

\subsection{Conclusions}

This thesis investigated the performance and viability of using toroidal configurations of confined magnetic fields as an active method of radiation shielding, and compared those results with the effectiveness of several configurations of passive material shields. The effectiveness of these methods was determined by evaluating each shielding configuration's effectiveness at reducing the energy absorbed by a payload when bombarded by protons with energies ranging from $1 \mathrm{MeV}$ to $20 \mathrm{GeV}$, and weighting the effectiveness against each energy level with the flux values of protons across that energy spectrum for both the Solar Particle Event and Galactic Cosmic Ray radiation environments.

To contextualize the effectiveness of electrodynamic toroidal shielding, the results were compared with those of passive material shielding. A $1 \mathrm{~cm}$-thick shield of water was calculated to reduce the radiation dose from SPE protons by $86 \%$, and negligibly affect the dose from GCR protons. A $10 \mathrm{~cm}$-thick shield of water reduced the SPE dose by $94 \%$, but the GCR dose by only $1 \%$. Passive bulk material shielding composed of water was shown to be an adequate and effective shield against solar particle radiation, but ineffective against Galactic Cosmic Rays.

Several configurations of toroidal shielding were investigated. The first of the two most effective configurations surrounded the cylindrical candidate spacecraft with 5 large toroids, each with a smaller nested toroid inside, and which included one additional toroidal shield on each of the cylindrical spacecraft's endcaps (configuration 
C). With interior magnetic fields of approximately 1.7 Tesla, this shielding reduced SPE radiation dose by $87 \%$ and the GCR dose by $11 \%$. The second configuration consisted of 10 small primary toroids surrounding the spacecraft with one additional toroidal shield on each of the spacecraft's endcaps (configuration E). At the same interior magnetic field strength of 1.7 Tesla, this configuration reduced the SPE radiation dose by $85 \%$ and the GCR dose by $10 \%$. With magnetic field strengths of approximately 1.7 Tesla, these configurations of electrodynamic toroidal shielding provide significant SPE dose reduction, but are minimally effective against GCRs.

However, increasing the magnetic field strength by a factor of 10, yielding a magnetic field strength of approximately 17 Tesla, vastly improves the viability of electrodynamic toroidal shielding against Galactic Cosmic Rays. At this magnetic field strength, configuration C provides a $90 \%$ SPE dose reduction, but more importantly is able to reduce the radiation dose from GCRs by $76 \%$. Configuration E, at this magnetic field strength, reduces SPE dose by $90 \%$, and reduces GCR dose by $61 \%$. By increasing the magnetic field strengths involved, toroidal shielding is able to provide substantial protection against Galactic Cosmic Rays - far greater than what the same mass of material shielding would be able to provide.

The above two toroidal shield configurations were also tested as combination shielding configurations - where a $1 \mathrm{~cm}$-thick shield of water was also added around the spacecraft. Even at the nominal magnetic field strengths of 1.7 Tesla, these combined configurations provided very high levels of protection against Solar Particle Events. $\mathrm{C}^{*}$ reduced the SPE dose by $95 \%$ and GCR dose by $14 \%$, and $\mathrm{E}^{*}$ reduced the SPE dose by $93 \%$ and the GCR dose by $12 \%$. By increasing the magnetic field strengths up to 17 Tesla, the dose reductions are further increased. At these magnetic field strengths, $\mathrm{C}^{*}$ experienced a $97 \%$ SPE dose reduction and a $74 \%$ GCR dose reduction, while $\mathrm{E}^{*}$ experienced a $96 \%$ SPE dose reduction and a 57\% GCR dose reduction. 
Passive material shielding was shown to provide substantial shielding against Solar Particle Event radiation, but is minimally effective against Galactic Cosmic Rays with shielding thinner than approximately $10 \frac{\mathrm{g}}{\mathrm{cm}^{2}}$. Electrodynamic confined magnetic toroidal shielding at magnetic field strengths of 1.7 Tesla was shown to be substantially effective against SPEs, but also minimally effective against GCRs. Combining the toroidal shielding configurations with a $1 \mathrm{~cm}$-thick shield of water was very effective against SPEs, but minimally effective against GCRs. However, the same pure toroidal and combined toroidal/water shielding with 10 times that magnetic field strength were shown to be very effective at reducing the radiation dose from GCR protons.

Electrodynamic shielding is the most complex of the three primary types of radiation shielding: bulk material, electrostatic, and electrodynamic. While the radiation shielding used on spacecraft today falls into the bulk material category, it is only moderately effective against Solar Particle Events and ineffective against Galactic Cosmic Rays. To sufficiently protect against both SPEs and GCRs without adding tens of thousands of kilograms of mass for shielding, the use of toroidal solenoids to generate confined magnetic fields was shown to be an effective option.

There are numerous challenges involved in such a system, particularly at the higher magnetic field strengths tested, such as preparing the superconductive wires and cooling the system to below critical temperature. If these challenges can be surmounted, the active shielding systems discussed in this thesis could be the systems that enable crewed exploration into interplanetary space. 


\subsection{Future Work}

The simulation program that was developed for this thesis sought to identify general patterns about the effectiveness of electrodynamic toroidal shielding in reducing the radiation dose experienced by spacecraft. Because the radiation dose absorbed when an energetic particle impacts an object is highly dependent on the material(s) passed and the corresponding path length(s), the results gathered in this thesis may be significantly different from those gathered using a similar program but with a different spacecraft configuration.

This thesis took several simplifying assumptions that could affect the viability of constructing an actual system. The two primary factors to consider for a more detailed system viability analysis include the power required to keep the solenoids below the superconductor critical temperature and maintain the high current level, and the control or negation of undesired residual magnetic field in the habitable volume of the spacecraft. Furthermore, future detailed investigations should examine the need, or possible lack thereof, for support structures to keep the toroidal shields at optimal shape.

To gather accurate results for a relatively low computational cost, all of the several thousand particles generated per simulation were made to travel towards the payload, and were therefore either successfully deflected, or impacted the payload. However, to best model the effectiveness of a shielding system that deflects particles, one must also simulate a similarly large number of particles that are made to travel near the payload, but not directly impact it. By doing this, the effectiveness of each shielding configuration at reducing radiation dose will also take into account particles that impacted the payload because of the shielding system that would not have otherwise done so. 
To accurately determine the effectiveness and viability of electrodynamic toroidal shielding or other types of radiation shielding on a specific mission such as a journey to the Moon or to Mars, the simulation program should accurately model the mission's spacecraft, and the location of all its components and inhabitants. To accurately calculate the radiation dose absorbed by inhabitants of a spacecraft, specific biological radiation transport models must be used, as the absorption of radiation in humans and animals is substantially more complicated than simple energy deposition. Furthermore, secondary particle effects such as muon generation should also be considered, as secondary particles often are more hazardous than their corresponding primary particles. Performing similar analysis on a much higher-fidelity spacecraft model, and taking into account the above considerations, the use of an energetic particle simulation framework such as Geant4 is recommended.

The simulations run for this thesis only included protons, however protons make up only a portion of the Solar Particle Event and Galactic Cosmic Ray radiation spectra. Future simulations seeking to accurately evaluate the effectiveness of specific radiation shielding systems should be sure to evaluate that system's effectiveness against the many ions and other particles that contribute to the radiation dose experienced in space. 
Bibliography

[1] Space Faring: The Radiation Challenge. 2008. URL: https://www . nasa.gov/ pdf/284273main_Radiation_HS_Mod1.pdf.

[2] "Radiation Exposure Comparisons with Mars Trip Calculation". In: (2013). URL: https://www . nasa.gov/jpl/msl/mars-rover-curiosity-pia17601 . html.

[3] Francis A. Cucinotta. "Radiation Risk Acceptability and Limitations". In: (2010). URL: https://three.jsc.nasa.gov/articles/astronautradlimitsfc.pdf.

[4] "The early years of radiation protection: a tribute to Madame Curie". In: Canadian Medical Association Journal 159 (1998), pp. 1389-1391.

[5] Space Radiation Guide. Alexandria, 1962.

[6] Nathan Gehrke. "Utilizing Permanent On-Board Water Storage for Efficient Deep Space Radiation Shielding". MA thesis. California Polytechnic State University, San Luis Obispo, 2018.

[7] Some Aspects of Active Shielding Against The Radiation In Space. Tech. rep. 1971.

[8] Reiner B. Meinke Erwan A. Kervendal Daniel R. Kirk. "Spacecraft Radiation Shielding Using Ultralightweight Superconducting Magnets". In: Journal of Spacecraft and Rockets 46.5 (Sept. 2009). DOI: 10.2514/1.37490.

[9] Revolutionary Concepts of Radiation Shielding for Human Exploration of Space. Tech. rep. 213688. NASA; Marshall Space Flight Center, 2005.

[10] Radiation Basics. 2017. URL: https://www.nrc.gov/about-nrc/radiation/ health-effects/radiation-basics.html. 
[11] Relativistic Energy. URL: http://hyperphysics.phy-astr.gsu.edu/hbase/ Relativ/releng.html.

[12] Kenneth A. LaBel. Radiation Effects on Electronics 101. 2004. URL: https: //nepp . nasa . gov / DocUploads / 392333B0 - 7A48-4A04-A3A72B0B1DD73343/ Rad_Effects_101_WebEx.pdf.

[13] Ionizing radiation, health effects and protective measures. 2016. URL: http: //www . who . int/news-room/fact-sheets/detail/ionizing-radiationhealth-effects-and-protective-measure.

[14] Encyclopedia of Science and Technology. 8th ed. Vol. 16. McGraw-Hill, 1997.

[15] Contribution of High Charge and Energy (HZE) Ions During Solar-Particle Event of September 29, 1989. 1989.

[16] "Interplanetary Crew Dose Rates for the August 1972 Solar Particle Event". In: Radiation Research 153 (2000), pp. 729-733. ISSN: 0033-7587/00. URL: http: //emmrem.unh.edu/papers/parsons.pdf.

[17] Help: Solar proton models. 2018. URL: https ://www. spenvis.oma.be/help/ background/flare/flare.html\#SFLUX.

[18] "STUDY OF LONG-TERM COSMIC RAY VARIABILITY DURING SOLAR CYCLE 22 TO 24". In: Indian Journal of Scientific Research (2018).

[19] Help: GCR particle models. 2018. URL: https://www.spenvis.oma.be/help/ background/gcr/gcr.html.

[20] "Radiation Hazards on Space Missions Outside the Magnetosphere". In: Advances in Space Research 9.10 (1989), pp. 285-291.

[21] "Configuration studies for active electrostatic space radiation shielding". In: Acta Astronautica 88 (2013), pp. 138-145. 
[22] Carl R. Nave. Toroidal Magnetic Field. URL: http://hyperphysics . phyastr.gsu.edu/hbase/magnetic/solenoid.html.

[23] Eric W. Weisstein. Sphere Point Picking. URL: http://mathworld. wolfram. com/SpherePointPicking.html.

[24] "Microstructural evolution of the BSCCO-2223 during powder-in-tube processing". In: Journal of Materials Research 9 (11 1994), pp. 2789-2808.

[25] "Synthesis and superconducting properties of bscco including precipitates with high density". In: Phase Transitions 42 (1-2 1993), pp. 47-51.

[26] Carl R. Nave. Toroidal Magnetic Field. URL: http ://hyperphysics . phyastr.gsu.edu/hbase/magnetic/toroid.html.

[27] Bragg Curves and Peaks. 2018. URL: https://www.bnl.gov/nsrl/userguide/ bragg-curves-and-peaks . php.

[28] PSTAR. 2018. URL: https://physics.nist.gov/PhysRefData/Star/Text/ PSTAR.html.

[29] Juan A. Aguilar. Particle Astrophysics Lecture 3. URL: http://w3. i ihe.ac . be/ aguilar/PHYS-467/PA3.pdf.

[30] "Towards a $20 \mathrm{kA}$ high temperature superconductor current lead module using REBCO tapes". In: Superconductor Science and Technology 31.1 (2017). URL: http://iopscience.iop.org/article/10.1088/1361-6668/aa9b09/meta. 